TRANSACTIONS OF THE

AMERICAN MATHEMATICAL SOCIETY

Volume 362, Number 9, September 2010, Pages 4983-5042

S 0002-9947(10)05154-8

Article electronically published on April 14, 2010

\title{
THE HOMOTOPY FIXED POINT SPECTRA OF PROFINITE GALOIS EXTENSIONS
}

\author{
MARK BEHRENS AND DANIEL G. DAVIS
}

\begin{abstract}
Let $E$ be a $k$-local profinite $G$-Galois extension of an $E_{\infty}$-ring spectrum $A$ (in the sense of Rognes). We show that $E$ may be regarded as producing a discrete $G$-spectrum. Also, we prove that if $E$ is a profaithful $k$-local profinite extension which satisfies certain extra conditions, then the forward direction of Rognes's Galois correspondence extends to the profinite setting. We show that the function spectrum $F_{A}\left(\left(E^{h H}\right)_{k},\left(E^{h K}\right)_{k}\right)$ is equivalent to the localized homotopy fixed point spectrum $\left((E[[G / H]])^{h K}\right)_{k}$, where $H$ and $K$ are closed subgroups of $G$. Applications to Morava $E$-theory are given, including showing that the homotopy fixed points defined by Devinatz and Hopkins for closed subgroups of the extended Morava stabilizer group agree with those defined with respect to a continuous action in terms of the derived functor of fixed points.
\end{abstract}

\section{Contents}

1. Introduction

2. Discrete symmetric $G$-spectra

3. Homotopy fixed points of discrete $G$-spectra 4994

4. Continuous $G$-spectra 5002

5. Modules and commutative algebras of discrete $G$-spectra 5004

6. Profinite Galois extensions 5013

7. Closed homotopy fixed points of profinite Galois extensions 5021

8. Applications to Morava E-theory 5033

Acknowledgments $\quad 5040$

References 5041

\section{INTRODUCTION}

In [34, John Rognes develops a Galois theory of commutative $S$-algebras which mimics the Galois theory of commutative rings. Let $k$ be an $S$-module, and let $(-)_{k}$ denote Bousfield localization with respect to $k$. Given a $k$-local cofibrant

Received by the editors August 6, 2008 and, in revised form, July 3, 2009.

2010 Mathematics Subject Classification. Primary 55P43; Secondary 55P91, 55 Q51.

The first author was supported by NSF grant DMS-0605100, the Sloan Foundation, and DARPA.

Part of the second author's work on this paper was supported by an NSF VIGRE grant at Purdue University, a visit to the Mittag-Leffler Institute, and a grant from the Louisiana Board of Regents Support Fund.

(C)2010 American Mathematical Society Reverts to public domain 28 years from publication 
commutative $S$-algebra $A$, and a cofibrant commutative $A$-algebra $E$ that is $k$ local, Rognes gives the following definition of a finite $k$-local Galois extension.

Definition 1.0.1 (Finite Galois extension). The spectrum $E$ is a $k$-local $G$-Galois extension of $A$, for a finite discrete group $G$ if it satisfies the following conditions:

(1) $G$ acts on $E$ through commutative $A$-algebra maps.

(2) The canonical map $A \rightarrow E^{h G}$ is an equivalence.

(3) The canonical map $\left(E \wedge_{A} E\right)_{k} \rightarrow \operatorname{Map}(G, E)$ is an equivalence.

$E$ is said to be $k$-locally faithful over $A$ if $\left(M \wedge_{A} E\right)_{k} \simeq *$ implies that $M_{k} \simeq *$ for every $A$-module $M$. In the context of $k$-local Galois extensions, we shall simply refer to such extensions as faithful.

Remark 1.0.2. Rognes (34, Prop. 6.3.3]; see also [1]) shows that a $k$-local $G$-Galois extension is faithful if and only if the additive form of Hilbert's Theorem 90 holds:

$$
\left(E^{t G}\right)_{k} \simeq *
$$

We will mostly consider faithful Galois extensions, because these are the Galois extensions for which the fundamental theorem of Galois theory holds. We refer the interested reader to [35] for an example, due to Ben Wieland, of a Galois extension that is not faithful.

Let $G$ be a profinite group. Following (and slightly modifying) Rognes's definition [34, Def. 8.1.1] of a $k$-local pro- $G$-Galois extension, we define a (profaithful) $k$-local profinite $G$-Galois extension $E$ of $A$ to be a colimit (in the category of commutative $A$-algebras) of (faithful) $k$-local $G / U_{\alpha}$-Galois extensions $E_{\alpha}$ of $A$, for a cofinal system of open normal subgroups $U_{\alpha}$ of $G$ (see Definition 6.2.1). Since a colimit of $k$-local spectra need not be $k$-local, the spectrum $E$ is not necessarily $k$-local.

In [6], the second author developed a category of discrete $G$-spectra and defined their homotopy fixed points (see also [41, 24, [32, 14], 26]). In this paper, we examine $k$-local profinite $G$-Galois extensions $E$ of $A$ as objects in the category of discrete $G$-spectra, and we study the spectra of $A$-module maps between the various homotopy fixed point spectra of $E$. Unfortunately, to say meaningful things it seems that we must impose more hypotheses on our profinite Galois extensions.

Assumption 1.0.3. In this paper, we shall only concern ourselves with localizations $(-)_{k}$ which are given as a composite of two localization functors $\left((-)_{T}\right)_{M}$, where $(-)_{T}$ is a smashing localization and $(-)_{M}$ is a localization with respect to a finite spectrum $M$. The spectra $S, H \mathbb{F}_{p}, E(n)$, and $K(n)$ are all examples of such localizations $k$ (see [4], 19]).

For a cofibrant commutative $S$-algebra $B$ and a cofibrant commutative $B$-algebra $C$, the $k$-local Amitsur derived completion $B_{k, C}^{\wedge}$ is the homotopy limit of the cosimplicial spectrum

(see, for example, [34, Def. 8.2.1]).

$$
C_{k} \Rightarrow\left(C \wedge_{B} C\right)_{k} \Rightarrow\left(C \wedge_{B} C \wedge_{B} C\right)_{k} \cdots
$$

Definition 1.0.4. Let $E$ be a $k$-local profinite $G$-Galois extension of $A$.

(1) The extension $E$ is consistent if the coaugmentation of the $k$-local Amitsur derived completion

$$
A \rightarrow A_{k, E}^{\wedge}
$$

is an equivalence. 
(2) The extension $E$ is of finite virtual cohomological dimension (finite vcd) if the profinite group $G$ has finite vcd (i.e., $G$ has an open subgroup $U$ of finite cohomological dimension: there exists a $d$ such that $H_{c}^{s}(U ; M)=0$ for each $s>d$ and each discrete $U$-module $M$ ).

Assumption 1.0.3 ensures that if $E$ has finite vcd, then the condition of $E$ being consistent is equivalent to requiring that the map

$$
A \rightarrow\left(E^{h G}\right)_{k}
$$

is an equivalence. This is proven as Corollary 6.3.2

It then follows that the maps

$$
E_{\alpha} \rightarrow\left(E^{h U_{\alpha}}\right)_{k}
$$

are equivalences (Lemma 6.3.6). The consistency hypothesis may be unnecessary, since we do not know of any profinite Galois extensions which are not consistent.

The main concern of this paper is the study of the intermediate homotopy fixed point spectra $E^{h H}$ with respect to closed subgroups $H$ of $G$. We prove the "forward" direction of the Galois correspondence.

Theorem (7.2.1). Suppose that $E$ is a consistent profaithful $k$-local profinite $G$ Galois extension of $A$ of finite vcd and that $H$ is a closed subgroup of $G$.

(1) The spectrum $E$ is $k$-locally $H$-equivariantly equivalent to a consistent profaithful $k$-local $H$-Galois extension of $\left(E^{h H}\right)_{k}$ of finite vcd.

(2) If $H$ is a normal subgroup of $G$, then the spectrum $E^{h H}$ is $k$-locally equivalent to a profaithful $k$-local $G / H$-Galois extension of $A$. If the quotient $G / H$ has finite vcd, then this extension is consistent (and of finite vcd) over A.

Remark 1.0.5. Note that the open subgroups of a profinite group $G$ are precisely the closed subgroups of finite index. Also, if $G$ has finite vcd, then it easily follows from [38, I.3.3] that every closed subgroup also has finite vcd.

We also identify the function spectrum of $A$-module maps between any two such homotopy fixed point spectra.

Theorem (7.3.1). Let $E$ be a consistent profaithful $k$-local profinite $G$-Galois extension of finite $v c d$, and let $H$ and $K$ be closed subgroups of $G$. Then there is an equivalence

$$
F_{A}\left(\left(E^{h H}\right)_{k},\left(E^{h K}\right)_{k}\right) \simeq\left((E[[G / H]])^{h K}\right)_{k} .
$$

The spectrum $E[[G / H]]$ that appears on the right-hand side of (1.1) is the continuous $G$-spectrum with the diagonal action. The case where $K=H=\{e\}$ was handled by Rognes [34, (8.1.3)].

In the context of Morava $E$-theory, (1.1) was proven in [15] under the additional assumption that $K$ is finite, and it was suggested by the authors of [15] that (1.1) should be true with this extra assumption removed. Another source of motivation for this work arises from the fact that a special case of (1.1) (Corollary 7.3.2) was needed in an essential way by the first author in [2] (see [2, Thm. 2.3.2, Cor. 2.3.3]).

One important example of a profinite Galois extension is given by Morava $E$ theory. Let $k=K(n)$ be the $n$th Morava $K$-theory spectrum and let $A=S_{K(n)}$ be the $K(n)$-local sphere spectrum. Let $G=\mathbb{G}_{n}$ be the $n$th extended Morava stabilizer group $\mathbb{S}_{n} \rtimes \operatorname{Gal}\left(\mathbb{F}_{p^{n}} / \mathbb{F}_{p}\right)$. Let $E_{n}$ be the $n$th Morava $E$-theory spectrum, 
where $\left(E_{n}\right)_{*}=W\left(\mathbb{F}_{p^{n}}\right)\left[\left[u_{1}, \ldots, u_{n-1}\right]\right]\left[u^{ \pm 1}\right]$. Goerss and Hopkins [16, building on the work of Hopkins and Miller [33, have shown that $\mathbb{G}_{n}$ acts on $E_{n}$ by maps of commutative $S$-algebras. Devinatz and Hopkins [9] have given constructions of homotopy fixed point spectra $E_{n}^{d h H}$ for closed subgroups $H$ of $\mathbb{G}_{n}$. In particular, they show that there is an equivalence

$$
E_{n}^{d h \mathbb{G}_{n}} \simeq S_{K(n)}
$$

Thus, the homotopy fixed point spectra of $E_{n}$ are intimately related to the $n$th chromatic layer of the sphere spectrum.

Rognes [34, Thm. 5.4.4, Prop. 5.4.9] proved, for $U$ an open normal subgroup of $\mathbb{G}_{n}$, that the work of Devinatz and Hopkins [8, 9] shows that $E_{n}^{d h U}$ is a faithful $K(n)$-local $\mathbb{G}_{n} / U$-Galois extension of $S_{K(n)}$. Therefore, the discrete $\mathbb{G}_{n}$-spectrum

$$
F_{n}=\operatorname{colim}_{U \unlhd_{o} \mathbb{G}_{n}} E_{n}^{d h U}
$$

is a profaithful $K(n)$-local profinite $\mathbb{G}_{n}$-Galois extension of $S_{K(n)}$. Additionally, the profinite extension $F_{n}$ of $S_{K(n)}$ is consistent and has finite vcd (Proposition 8.1.2). The spectrum $E_{n}$ is recovered by the equivalence 9 ]

$$
E_{n} \simeq\left(F_{n}\right)_{K(n)}
$$

As mentioned above, for any closed subgroup $H$ of $\mathbb{G}_{n}$, Devinatz and Hopkins [9] constructed the commutative $S$-algebra $E_{n}^{d h H}$. Further, they showed that $E_{n}^{d h H}$ behaves like a homotopy fixed point spectrum with respect to a continuous action of $H$. In more detail, 9] showed that $E_{n}^{d h H}$ has the following properties: (a) there is a $K(n)$-local $E_{n}$-Adams spectral sequence

$$
H_{c}^{s}\left(H ; \pi_{t}\left(E_{n}\right)\right) \Rightarrow \pi_{t-s}\left(E_{n}^{d h H}\right),
$$

where the $E_{2}$-term is the continuous cohomology of $H$, with coefficients in the profinite $H$-module $\pi_{t}\left(E_{n}\right)$, and this spectral sequence has the form of a descent spectral sequence; (b) when $H$ is finite, there is a weak equivalence $E_{n}^{d h H} \rightarrow E_{n}^{h H}$, and the descent spectral sequence for $E_{n}^{h H}$ is isomorphic to the spectral sequence in (a); and (c) $E_{n}^{d h H}$ is an $(N(H) / H)$-spectrum, where $N(H)$ is the normalizer of $H$ in $\mathbb{G}_{n}$.

On the other hand, when $H$ is not finite, $E_{n}^{d h H}$ is not known to actually be the $H$-homotopy fixed point spectrum of $E_{n}$, because (a) it is not constructed with respect to a continuous $H$-action, and (b) it is not obtained by taking the total right derived functor of fixed points (and homotopy fixed points are, by definition, the total right derived functor of fixed points, in some sense; see [6, Remark 8.4] for the precise definition in the case of a continuous $H$-spectrum that arises from a tower of discrete $H$-spectra). To address this situation, in [6], the second author showed that $H$ does act continuously on $E_{n}$ and there is an actual $H$-homotopy fixed point spectrum $E_{n}^{h H}$, with a descent spectral sequence

$$
H_{c}^{s}\left(H ; \pi_{t}\left(E_{n}\right)\right) \Rightarrow \pi_{t-s}\left(E_{n}^{h H}\right)
$$

From the above discussion, we see from the properties of $E_{n}^{d h H}$ and $E_{n}^{h H}$ that they should be equivalent to each other, and by a result in the second author's thesis [5], they are. However, since this part of [5] was never published, we use the machinery of this paper to prove the equivalence of these two spectra. In more detail, we give proofs of the following two results (which originally appeared in [5]). 
Theorem (8.2.1). For every closed subgroup $H$ of $\mathbb{G}_{n}$, there is an equivalence

$$
E_{n}^{d h H} \simeq E_{n}^{h H}
$$

between the Devinatz-Hopkins construction and the homotopy fixed points that are defined with respect to the continuous action of $H$.

The above theorem shows that $E_{n}^{d h H}$ can be referred to as a homotopy fixed point spectrum, whereas, previously, $E_{n}^{d h H}$ was only known to behave like a homotopy fixed point spectrum.

Theorem (8.2.3, 8.2.4, 8.2.5). Let $H$ be a closed subgroup of $\mathbb{G}_{n}$ and let $X$ be a finite spectrum. Then there is an equivalence

$$
E_{n}^{d h H} \wedge X \simeq\left(E_{n} \wedge X\right)^{h H}
$$

and the $K(n)$-local $E_{n}$-Adams spectral sequence for $\pi_{*}\left(E_{n}^{d h H} \wedge X\right)$ is isomorphic to the descent spectral sequence for $\pi_{*}\left(\left(E_{n} \wedge X\right)^{h H}\right)$ from the $E_{2}$-terms onward. In particular,

$$
(X)_{K(n)} \simeq\left(E_{n} \wedge X\right)^{h \mathbb{G}_{n}} .
$$

The paper is organized as follows. Our notion of homotopy fixed point spectra uses the framework of equivariant spectra (with respect to a profinite group) as developed by the second author [6]. The foundations in [6] use Bousfield-Friedlander spectra. Since we need to work with structured ring spectra to do Galois theory, it is essential for this paper that we reformulate portions of [6] in the context of symmetric spectra. A concise summary of these foundations appears in Section 2 In Section 3, we describe properties of the homotopy fixed point functor. In Section 4, we describe continuous $G$-spectra, generalizing somewhat the setting of $[6]$. In Section 5, we explain how to extend our constructions to categories of modules and commutative algebras of spectra. In Section [ we explain how profinite Galois extensions give rise to discrete $G$-spectra, and we show that the homotopy fixed points with respect to open subgroups of the Galois group give rise to intermediate finite Galois extensions. In Section 7 , we prove our results concerning the homotopy fixed point spectra with respect to closed subgroups of the Galois group. In Section 8, we show that the hypotheses on profinite Galois extensions which we require are satisfied by Morava $E$-theory. We then apply our machinery to show that the Devinatz-Hopkins homotopy fixed points agree with the second author's homotopy fixed points and deduce some corollaries.

\section{Discrete Symmetric $G$-SPeCtra}

Let $G$ be a profinite group. We begin this section by describing the basic categories of discrete $G$-objects that will be used in this paper. We then describe and compare the model structures on the categories of discrete $G$-objects in BousfieldFriedlander and symmetric spectra. We end this section with descriptions of some basic constructions in the category of discrete $G$-spectra. More detailed accounts of some of these model categories and constructions can be found in [6].

2.1. Simplicial discrete $G$-sets. A $G$-set $Z$ is said to be discrete if, for every element $z \in Z$, the stabilizer $\operatorname{Stab}_{G}(z)$ is open in $G$. We may express this condition by saying that $Z$ is the colimit of its fixed points:

$$
Z=\operatorname{colim}_{U \leq_{o} G} Z^{U},
$$


where the colimit is taken over all open subgroups. These conditions are equivalent to the condition that the action map $G \times Z \rightarrow Z$ is continuous, when $Z$ is given the discrete topology. A simplicial discrete $G$-set is a simplicial object in the category of discrete $G$-sets.

Goerss showed that the category $s \operatorname{Set}_{G}$ of simplicial discrete $G$-sets admits a model category structure [14].

Theorem 2.1.1 (Goerss). The category $s \operatorname{Set}_{G}$ admits a model category structure, where

- the cofibrations are the monomorphisms,

- the weak equivalences are those morphisms which are weak equivalences on underlying simplicial sets,

- the fibrations are determined.

Lemma 2.1.2. The model structure on $s \operatorname{Set}_{G}$ is left proper and cellular.

Proof. The model structure is left proper because the cofibrations and weak equivalences are precisely the cofibrations and weak equivalences on the underlying simplicial sets, and the model category structure on simplicial sets is left proper. The model structure in [14] is cofibrantly generated, with generating cofibrations $I$ and generating trivial cofibrations $J$, where:

$$
\begin{aligned}
& I=\left\{G / U \times \partial \Delta^{n} \hookrightarrow G / U \times \Delta^{n}: U \leq_{o} G, n \geq 0\right\}, \\
& J=\left\{A \stackrel{j}{\rightarrow} B: \begin{array}{l}
j \text { is a trivial cofibration } \\
\# B \leq \alpha
\end{array}\right\} .
\end{aligned}
$$

Here, $\alpha$ is a fixed infinite cardinal greater than the cardinality of $G$ and \# $B$ denotes the cardinality of the set of nondegenerate simplices of $B$ (see the proof of Lemma 1.13 of [14]). The axioms of being cellular are immediately verified from this description of the generating cofibrations.

The category $\left(s \operatorname{Set}_{G}\right)_{*}$ of pointed simplicial discrete $G$-sets, being an undercategory, inherits a model structure from $s \operatorname{Set}_{G}$. The cofibrations, weak equivalences, and fibrations are detected on the level of underlying simplicial discrete $G$-sets. If $K$ and $L$ are pointed simplicial discrete $G$-sets, then their smash product

$$
K \wedge L
$$

is easily seen to be a simplicial discrete $G$-set. The smash product gives the category $\left(s \operatorname{Set}_{G}\right)_{*}$ a symmetric monoidal structure. (It does not extend to a closed symmetric monoidal structure.)

Lemma 2.1.3. The model category structure on $\left(s \operatorname{Set}_{G}\right)_{*}$ is left proper and cellular. With respect to the symmetric monoidal structure given by the smash product, the model category $\left(s \operatorname{Set}_{G}\right)_{*}$ is a symmetric monoidal model category.

Proof. Left properness follows from the fact that $s \operatorname{Set}_{G}$ is left proper. The model structure on $\left(s \operatorname{Set}_{G}\right)_{*}$ is cofibrantly generated with generating cofibrations (respectively generating trivial cofibrations) $I_{+}$(respectively $J_{+}$). Here, $I_{+}$and $J_{+}$are the sets of maps obtained from $I$ and $J$ by adding a disjoint basepoint on which $G$ acts trivially. The axioms of being a symmetric monoidal model category are easily verified. 
2.2. Discrete $G$-spectra. Define the category of discrete $G$-spectra $\operatorname{Sp}_{G}$ to be the category of Bousfield-Friedlander spectra of simplicial discrete $G$-sets. An object $X \in \operatorname{Sp}_{G}$ consists of a sequence $\left\{X_{i}\right\}_{i \geq 0}$, where each $X_{i}$ is a pointed simplicial discrete $G$-set, together with $G$-equivariant maps

$$
\sigma_{i}: S^{1} \wedge X_{i} \rightarrow X_{i+1} .
$$

Here, $S^{1}$ is given the trivial $G$-action.

A map $f: X \rightarrow Y$ of discrete $G$-spectra is a sequence of $G$-equivariant maps of pointed simplicial sets $f_{i}: X_{i} \rightarrow Y_{i}$ which are compatible with the spectrum structure maps.

In [6], the second author studied the following model structure.

Theorem 2.2.1. The category $\mathrm{Sp}_{G}$ admits a model structure, where

- the cofibrations are the cofibrations of underlying Bousfield-Friedlander spectra,

- the weak equivalences are the stable weak equivalences of the underlying Bousfield-Friedlander spectra,

- the fibrations are determined.

The method used in [6] was to transport a Jardine model structure on presheaves of spectra on an appropriate site. However, an alternative approach is given below using the machinery of M. Hovey [20].

Proof. Observe that $\left(s \operatorname{Set}_{G}\right)_{*}$ satisfies the conditions of Definition 3.3 of 20 . Therefore, the category $\mathrm{Sp}_{G}$ of spectra of simplicial discrete $G$-sets admits a stable model category structure, where:

- The cofibrations are those morphisms $A \rightarrow B$ where the induced maps

$$
\begin{aligned}
A_{0} & \rightarrow B_{0}, \\
A_{i} \cup_{S^{1} \wedge A_{i-1}} S^{1} \wedge B_{i-1} & \rightarrow B_{i}, \quad n \geq 1,
\end{aligned}
$$

are cofibrations.

- The fibrant objects $X$ are those spectra for which

(1) the spaces $X_{i}$ are fibrant as simplicial discrete $G$-sets,

(2) the maps $X_{i} \rightarrow \Omega X_{i+1}$ are weak equivalences.

- The weak equivalences $f: X \rightarrow Y$ between fibrant objects are those $f$ for which the maps $f_{i}: X_{i} \rightarrow Y_{i}$ are all weak equivalences.

Clearly the cofibrations of $\mathrm{Sp}_{G}$ are the maps which are cofibrations of underlying Bousfield-Friedlander spectra. We are left with verifying that the weak equivalences in $\mathrm{Sp}_{G}$ are precisely the stable equivalences of underlying Bousfield-Friedlander spectra.

The forgetful functor

$$
\mathcal{U}: s \operatorname{Set}_{G} \rightarrow s \operatorname{Set}
$$

from simplicial discrete $G$-sets to simplicial sets is a left Quillen functor (it preserves cofibrations and trivial cofibrations and is left adjoint to the functor $\operatorname{CoInd}_{1}^{G}$ of Section 3.4). By Proposition 5.5 of [20], the induced forgetful functor

$$
\mathcal{U}: \mathrm{Sp}_{G} \rightarrow \mathrm{Sp}
$$

is a left Quillen functor. Let $(-)_{f G}$ denote the functorial fibrant replacement in $\mathrm{Sp}_{G}$, so that there are natural trivial cofibrations

$$
\alpha_{G, X}: X \rightarrow X_{f G}
$$


Suppose that $\phi: X \rightarrow Y$ is a morphism in $\operatorname{Sp}_{G}$ and consider the following diagram:

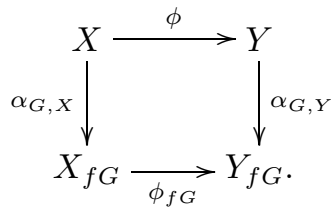

We claim that $\phi$ is a stable equivalence in $\mathrm{Sp}_{G}$ if and only if the induced morphism $\mathcal{U} \phi$ is a stable equivalence of underlying Bousfield-Friedlander spectra. Because $\mathcal{U}$ is a left Quillen functor, and the morphisms $\alpha_{G,-}$ are trivial cofibrations, we may conclude that the morphisms $\mathcal{U} \alpha_{G,-}$ induce stable equivalences of underlying Bousfield-Friedlander spectra.

Since the underlying spectrum of a fibrant object in $\mathrm{Sp}_{G}$ is fibrant in $\mathrm{Sp}$, and since the stable equivalences between fibrant objects in $\mathrm{Sp}_{G}$ and $\mathrm{Sp}$ are precisely the levelwise equivalences, we see that $\phi_{f G}$ is a stable equivalence if and only if $\mathcal{U} \phi_{f G}$ is a stable equivalence. Therefore, we may deduce that $\phi$ is a stable equivalence if and only if $\mathcal{U} \phi$ is a stable equivalence.

2.3. Discrete symmetric $G$-spectra. Let $\Sigma$ Sp denote the category of symmetric spectra (see [21, 29] for accounts of symmetric spectra). Define the category of discrete symmetric $G$-spectra $\Sigma \mathrm{Sp}_{G}$ to be the category of symmetric spectra of simplicial discrete $G$-sets. Let $\Sigma_{i}$ denote the $i$ th symmetric group. An object $X \in \Sigma \operatorname{Sp}_{G}$ consists of a sequence $\left\{X_{i}\right\}_{i \geq 0}$, where each $X_{i}$ is a pointed simplicial discrete $G \times \Sigma_{i}$-set, together with suitably compatible $G \times \Sigma_{i} \times \Sigma_{j}$-equivariant maps

$$
\sigma_{i, j}: S^{i} \wedge X_{j} \rightarrow X_{i+j}
$$

Here, $S^{i}=\left(S^{1}\right)^{\wedge i}$ is given the trivial $G$-action, and $\Sigma_{i}$ permutes the factors of the smash product $\left(S^{1}\right)^{\wedge i}$. When $G$ is finite, a discrete symmetric $G$-spectrum is simply a naïve symmetric $G$-spectrum, and not a genuine equivariant symmetric $G$-spectrum in the sense of $[28$.

Maps $f: X \rightarrow Y$ of discrete symmetric $G$-spectra are sequences of $G \times \Sigma_{i^{-}}$ equivariant maps of pointed simplicial sets $f_{i}: X_{i} \rightarrow Y_{i}$ which are compatible with the spectrum structure maps.

For a cofibrantly generated model category $\mathcal{C}$, let $\mathcal{C}^{\Sigma_{j}}$ denote the diagram category of $\Sigma_{j}$-equivariant objects in $\mathcal{C}$ with the projective model structure ([17, Thm. 11.6.1]).

Lemma 2.3.1. In the projective model category structure on $\left(s \operatorname{Set}_{G}\right)_{*}^{\Sigma_{j}}$ :

- the cofibrations are those maps that are projective cofibrations in the underlying category siet ${ }_{*}^{\Sigma_{j}}$,

- the weak equivalences are those maps that are weak equivalences in the underlying category siet ${ }_{*}^{\Sigma_{j}}$,

- the fibrations are determined.

Proof. The statement concerning weak equivalences follows immediately from the definition of the weak equivalences in the projective model structure. The projective cofibrations in $\left(s \operatorname{Set}_{G}\right)_{*}^{\Sigma_{j}}$ are generated by the set

$$
I_{+}^{\Sigma_{j}}=\left\{\left(\Sigma_{j} \times G / U \times \partial \Delta^{n}\right)_{+} \hookrightarrow\left(\Sigma_{j} \times G / U \times \Delta^{n}\right)_{+}: U \leq_{o} G, n \geq 0\right\} .
$$


Using the relative skeletal filtration, it is easy to see that the class of cofibrations generated by the set $I_{+}^{\Sigma_{j}}$ are the monomorphisms which are relative free $\Sigma_{j}$ complexes. However, these are precisely the projective cofibrations in ${s \mathrm{Set}_{*}}_{*}^{\Sigma_{j}}$.

Theorem 2.3.2. The category $\Sigma \mathrm{Sp}_{G}$ admits a left proper cellular model structure, where

- the cofibrations are the cofibrations of underlying symmetric spectra,

- the weak equivalences are the stable weak equivalences of underlying symmetric spectra,

- the fibrations are determined.

Proof. Observe that $\left(s \operatorname{Set}_{G}\right)_{*}$ satisfies the conditions of Definition 8.7 of 20]. Therefore, the category $\Sigma \operatorname{Sp}_{G}$ of symmetric spectra of simplicial discrete $G$-sets admits a stable model category structure, where:

- The cofibrations are those morphisms $A \rightarrow B$ where the induced maps

$$
\begin{aligned}
A_{0} & \rightarrow B_{0}, \\
A_{i} \cup_{L_{i} A} L_{i} B & \rightarrow B_{i}, \quad i \geq 1,
\end{aligned}
$$

are projective cofibrations in $\left(s \operatorname{Set}_{G}\right)_{*}^{\Sigma_{j}}$, where $L_{n}$ is the latching object of 20. Def. 8.4].

- The fibrant objects $X$ are those spectra for which

(1) the spaces $X_{i}$ are fibrant as simplicial discrete $G$-sets,

(2) the maps $X_{i} \rightarrow \Omega X_{i+1}$ are weak equivalences.

- The weak equivalences $f: X \rightarrow Y$ between fibrant objects are those $f$ for which the maps $f_{i}: X_{i} \rightarrow Y_{i}$ are all weak equivalences of underlying simplicial sets.

The cofibrations are immediately seen to be the cofibrations of underlying symmetric spectra, using Lemma 2.3.1. The verification that the weak equivalences are precisely the stable equivalences of underlying symmetric spectra is identical to the argument given in the proof of Theorem 2.2.1.

We have the following proposition, which helps to translate results in the category $\mathrm{Sp}_{G}$ to the category $\Sigma \mathrm{Sp}_{G}$.

Proposition 2.3.3. There is a Quillen equivalence

$$
\mathbb{V}_{G}: \mathrm{Sp}_{G} \rightleftarrows \Sigma \mathrm{Sp}_{G}: \mathbb{U}_{G},
$$

where $\mathbb{U}_{G}$ is the forgetful functor.

Proof. The functor $\mathbb{V}_{G}$ is the left adjoint of $\mathbb{U}_{G}$ : it is explicitly given by (see [21, Sec. 4.3])

$$
\mathbb{V}_{G}(X)=S \otimes_{T\left(\mathbb{G}_{1} S^{1}\right)} \mathbb{G} X,
$$

where $\mathbb{G} X$ is the symmetric sequence given by

$$
(\mathbb{G} X)_{i}=\left(\Sigma_{i}\right)_{+} \wedge X_{i}
$$

(where $G$ acts through its action on $X_{i}$ ), $\mathbb{G}_{1} S^{1}$ is the symmetric sequence

$$
\left(*, S^{1}, *, *, \cdots\right)
$$

(with trivial $G$-action), $T\left(\mathbb{G}_{1} S^{1}\right)$ is the free monoid on $\mathbb{G}_{1} S^{1}$ with respect to $\otimes$ (which gives the symmetric monoidal structure on symmetric sequences), and $S$ is 
the usual symmetric sequence $\left(S^{0}, S^{1}, S^{2}, \cdots\right)$. We have the following commutative diagram of functors:

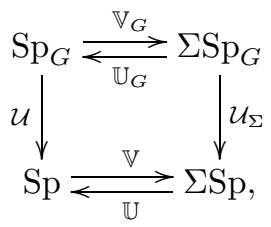

where the bottom row is the Quillen equivalence of [21, Sec. 4].

The functor $\mathbb{V}_{G}$ preserves cofibrations and trivial cofibrations because the functors $\mathcal{U}$ and $\mathcal{U}_{\Sigma}$ reflect and detect cofibrations and trivial cofibrations, and the functor $\mathbb{V}$ is a left Quillen functor. Therefore $\left(\mathbb{V}_{G}, \mathbb{U}_{G}\right)$ forms a Quillen pair.

To show that $\left(\mathbb{V}_{G}, \mathbb{U}_{G}\right)$ is a Quillen equivalence, we must show that for all cofibrant $X$ in $\operatorname{Sp}_{G}$ and all fibrant $Y$ in $\Sigma \mathrm{Sp}_{G}$, a morphism

$$
f: \mathbb{V}_{G} X \rightarrow Y
$$

is a weak equivalence if and only if its adjoint

$$
\tilde{f}: X \rightarrow \mathbb{U}_{G} Y
$$

is a weak equivalence. However, since the functors $\mathcal{U}$ and $\mathcal{U}_{\Sigma}$ reflect and detect weak equivalences, it suffices to show that:

$$
\left\{\begin{array}{c}
\mathcal{U}_{\Sigma} f: \mathbb{V} \mathcal{U} X \rightarrow \mathcal{U}_{\Sigma} Y \\
\text { is a weak equivalence }
\end{array}\right\} \quad \text { if and only if } \quad\left\{\begin{array}{c}
\mathcal{U} \tilde{f}: \mathcal{U} X \rightarrow \mathbb{U U}_{\Sigma} Y \\
\text { is a weak equivalence }
\end{array}\right\} .
$$

This follows from the fact that $\mathcal{U}$ preserves cofibrations (Theorem 2.2.1), $\mathcal{U}_{\Sigma}$ preserves fibrant objects (proof of Theorem 2.3 .2 ), and $(\mathbb{V}, \mathbb{U}$ ) form a Quillen equivalence [21, Thm. 4.2.5].

For the rest of this paper, we shall be working in the world of symmetric spectra and shall refer to a symmetric spectrum as simply a spectrum. For a spectrum $X$, we shall always use $\pi_{*} X$ to refer to its true homotopy groups (the maps $\left[S^{t}, X\right]$ in the stable homotopy category) and not the naïve homotopy groups (the colimit of the homotopy groups of $X_{i}$ ); see [36].

2.4. Mapping spectra. Let $K$ and $L$ be discrete $G$-sets. Then the set of (nonequivariant) functions $\operatorname{Map}(K, L)$ is a $G$-set with $G$ acting by conjugation. This action is given as follows: for $g \in G$ and $f \in \operatorname{Map}(K, L), g \cdot f$ is the map

$$
(g \cdot f)(z)=g f\left(g^{-1} z\right)
$$

Observe that $\operatorname{Map}(K, L)$ is not in general a discrete $G$-set, but it is if $K$ is finite.

For a finite set $K$ and a spectrum $X$, we define the mapping $\operatorname{spectrum} \operatorname{Map}(K, X)$ to be the spectrum whose $m$ th space is given by

$$
\operatorname{Map}(K, X)_{m}=\operatorname{Map}\left(K, X_{m}\right),
$$

where the $n$-simplices of $\operatorname{Map}\left(K, X_{m}\right)$ are the set $\operatorname{Map}\left(K,\left(X_{m}\right)_{n}\right)$. If $X$ is a discrete $G$-spectrum, and $K$ is a finite discrete $G$-set, then the above definitions combine to give that $\operatorname{Map}(K, X)$ is a discrete $G$-spectrum.

If $K=\lim _{\alpha} K_{\alpha}$ is a profinite set and $X$ is a spectrum, then the spectrum of continuous maps is the spectrum

$$
\operatorname{Map}^{c}(K, X)=\underset{\alpha}{\operatorname{colim}} \operatorname{Map}\left(K_{\alpha}, X\right) .
$$


If $K$ is a continuous $G$-space, with each $K_{\alpha}$ a discrete $G$-set, and if $X$ is a discrete $G$-spectrum, then $\operatorname{Map}^{c}(K, X)$ is a discrete $G$-spectrum.

Lemma 2.4.1. Let $K=\lim _{\alpha} K_{\alpha}$ be a profinite set, where each of the $K_{\alpha}$ is finite, and each of the maps $K_{\alpha} \rightarrow K_{\beta}$ in the pro-system is a surjection. The functor

$$
\operatorname{Map}^{c}(K,-): \Sigma \mathrm{Sp} \rightarrow \Sigma \mathrm{Sp}
$$

preserves stable equivalences.

Proof. In [37, it is shown that stable equivalences are preserved under finite products. The argument goes as follows: the canonical map from a finite wedge to a finite product is a $\pi_{*}$-isomorphism, hence a stable equivalence, and stable equivalences are preserved under finite wedges (this is easily checked from the definition of stable equivalences, by mapping into injective $\Omega$-spectra). Therefore, for each $\alpha$, the functor

$$
X \mapsto \operatorname{Map}\left(K_{\alpha}, X\right) \cong \prod_{K_{\alpha}} X
$$

preserves stable equivalences. Since we have assumed that the morphisms $K_{\alpha} \rightarrow K_{\beta}$ are surjections, the induced morphisms

$$
\operatorname{Map}\left(K_{\beta}, X\right) \rightarrow \operatorname{Map}\left(K_{\alpha}, X\right)
$$

are levelwise monomorphisms for every $X$.

The category of symmetric spectra possesses an injective stable model structure, where the injective cofibrations are the levelwise monomorphisms and the weak equivalences are the stable equivalences (see [21, p. 199]). The directed system

$$
\left\{\operatorname{Map}\left(K_{\alpha}, X\right)\right\}
$$

is a directed system of injective cofibrations between injectively cofibrant objects (every object is cofibrant in the injective model structure). The colimit

$$
\operatorname{Map}^{c}(K, X)=\underset{\alpha}{\operatorname{colim}} \operatorname{Map}\left(K_{\alpha}, X\right)
$$

may be computed on a cofinal $\lambda$-sequential subcategory of the indexing category of the system $\left\{K_{\alpha}\right\}$, for some ordinal $\lambda$. We deduce, by [17, Prop. 17.9.1], that the functor

$$
X \mapsto \operatorname{Map}^{c}(K, X)=\underset{\alpha}{\operatorname{colim}} \operatorname{Map}\left(K_{\alpha}, X\right)
$$

preserves stable equivalences.

If $A$ is an abelian group, endowed with the discrete topology, and $K=\lim _{\alpha} K_{\alpha}$ is a profinite set, the continuous maps $\operatorname{Map}^{c}(K, A)$ are given by

$$
\operatorname{Map}^{c}(K, A)=\underset{\alpha}{\operatorname{colim}} \operatorname{Map}\left(K_{\alpha}, A\right) .
$$

Lemma 2.4.2. Let $X$ be an object of $\Sigma \mathrm{Sp}$ and let $K$ be a profinite set satisfying the hypotheses of Lemma 2.4.1. Then there is an isomorphism

$$
\pi_{*} \operatorname{Map}^{c}(K, X) \cong \operatorname{Map}^{c}\left(K, \pi_{*}(X)\right)
$$

Proof. By Lemma 2.4.1 it suffices to assume that $X$ is fibrant. The result then follows from Corollary 5.3.2. 
2.5. Permutation spectra. Let $K$ be a discrete $G$-set. Then for $X$ a discrete $G$-spectrum, we may define the permutation spectrum $X[K]$ to be the spectrum whose $n$th space is given by

$$
X[K]_{n}=X_{n} \wedge K_{+} .
$$

We let $G$ act on the spectrum $X[K]$ through the diagonal action.

Lemma 2.5.1. The spectrum $X[K]$ is a discrete $G$-spectrum.

Proof. Note that

$$
\begin{aligned}
X_{n} \wedge K_{+} & \cong\left(\operatorname{colim}_{N \unlhd_{o} G} X_{n}^{N}\right) \wedge\left(\operatorname{colim}_{N^{\prime} \unlhd_{o} G} K_{+}^{N^{\prime}}\right) \\
& \cong \operatorname{colim}_{N, N^{\prime} \unlhd_{o} G}\left(X_{n}^{N} \wedge K_{+}^{N^{\prime}}\right)
\end{aligned}
$$

is a simplicial discrete $G$-set, with $G$ acting diagonally, since the simplicial set $X_{n}^{N} \wedge K_{+}^{N^{\prime}}$ has a diagonal $G /\left(N \cap N^{\prime}\right)$-action and the group $G /\left(N \cap N^{\prime}\right)$ is finite. Thus, the spectrum $X[K]$ is a discrete $G$-spectrum.

2.6. Smash products. Given discrete $G$-spectra $X$ and $Y$, we define their smash product

$$
X \wedge Y
$$

to be the smash product of the underlying symmetric spectra with $G$ acting diagonally. Since the smash product commutes with colimits, it follows, as in the proof of Lemma 2.5.1, that $X \wedge Y$ is a discrete $G$-spectrum. Also, if $K$ is a discrete $G$-set, then there is a $G$-equivariant isomorphism

$$
X \wedge S[K] \cong X[K]
$$

where the sphere spectrum $S$ has trivial $G$-action.

\section{Homotopy fixed points of Discrete $G$-SPeCtra}

Much of the material in this section is assembled from [41, [25], 14, [26], [32], [27, and [6]. Let $G$ be a profinite group. We begin this section with an account of the model category-theoretic definition of $G$-homotopy fixed points. We then describe the comparison with hypercohomology spectra. Finite index restriction and induction functors, as well as iterated homotopy fixed points for finite index subgroups are then discussed. We explain how continuous homomorphisms of groups induce various "change of group functors," of which induction, coinduction, fixed points, and restriction functors are all special cases. We then describe the various technical difficulties related to the homotopy fixed point construction for closed subgroups of $G$. The technical difficulties are observed to vanish if $G$ has finite cohomological dimension.

As alluded to above, Sections 3.3, 3.5, and 3.6 discuss the construction of iterated homotopy fixed points. Much of this material overlaps with portions of [7]: it was necessary to repeat some of the material from [7, so that certain issues are clear and to give a context for the results of Section 7.1 .

We note that, as explained in Section 2.3, "spectrum" means "symmetric spectrum," so that, for example, a "discrete $G$-spectrum" is a "discrete $G$-symmetric spectrum." 
3.1. The homotopy fixed point spectrum. For a discrete $G$-spectrum $X$, we define the fixed point spectrum by taking the fixed points levelwise:

$$
\left(X^{G}\right)_{i}=\left(X_{i}\right)^{G} \text {. }
$$

The $G$-fixed points functor is right adjoint to the functor triv, which associates to a spectrum $X$ the discrete $G$-spectrum $X$, where $X$ now has the trivial $G$-action:

$$
\text { triv }: \Sigma \mathrm{Sp} \rightleftarrows \Sigma \mathrm{Sp}_{G}:(-)^{G} \text {. }
$$

Lemma 3.1.1. The adjoint functors $\left(\right.$ triv,$\left.(-)^{G}\right)$ form a Quillen pair.

Proof. The functor triv preserves cofibrations and weak equivalences.

Let $\alpha_{G, X}: X \rightarrow X_{f G}$ denote a functorial fibrant replacement functor for the model category $\Sigma \mathrm{Sp}_{G}$, where $\alpha_{G, X}$ is a trivial cofibration of discrete $G$-spectra. The homotopy fixed point functor $(-)^{h G}$ is the Quillen right derived functor of $(-)^{G}$ and is thus given by

$$
X^{h G}=\left(X_{f G}\right)^{G} .
$$

3.2. Hypercohomology spectra. The functor $\Gamma_{G}=\operatorname{Map}^{c}(G,-)$ is a coaugmented comonad on the category of spectra, with coproduct

$$
\psi: \Gamma_{G}=\operatorname{Map}^{c}(G,-) \rightarrow \operatorname{Map}^{c}(G \times G,-) \cong \Gamma_{G} \circ \Gamma_{G}
$$

induced from the product on $G$, counit

$$
\Gamma_{G}=\operatorname{Map}^{c}(G,-) \rightarrow \operatorname{Map}^{c}(p t,-) \cong \mathrm{Id}
$$

induced from the unit on $G$, and coaugmentation

$$
\mathrm{Id} \rightarrow \operatorname{Map}^{c}(G,-)
$$

given by the inclusion of the constant maps.

Discrete $G$-spectra are coalgebras over the comonad $\Gamma_{G}$ (this follows from considering the map of spectra

$$
\begin{aligned}
X & \rightarrow \Gamma_{G}(X), \\
x & \mapsto(g \mapsto g \cdot x),
\end{aligned}
$$

for any discrete $G$-spectrum $X$ ).

Let $\mathcal{C}$ and $\mathcal{D}$ be categories, and suppose that $\Gamma$ is a comonad in $\mathcal{C}$. Dualizing Definition 9.4 of 30 , there is a notion of a $\Gamma$-functor

$$
F: \mathcal{C} \rightarrow \mathcal{D} \text {. }
$$

Let $Y$ be a $\Gamma$-coalgebra. Dualizing Construction 9.6 of [30], one may associate to $(F, \Gamma, Y)$ a cosimplicial object $C^{\bullet}(F, \Gamma, Y)$ in $\mathcal{D}$ (the comonadic cobar construction), given by

$$
C^{s}(F, \Gamma, Y)=F \Gamma^{s} Y .
$$

If $\Gamma$ is a coaugmented comonad, then the identity functor $\operatorname{Id}_{\mathcal{C}}$ is a $\Gamma$-functor. We will let $\Gamma^{\bullet} Y$ denote the cosimplicial object

$$
\Gamma^{\bullet} Y=C^{\bullet}\left(\operatorname{Id}_{\mathcal{C}}, \Gamma, Y\right)
$$

in $\mathcal{C}$.

In [6], the homotopy fixed point spectrum was shown to have the following alternate description, provided $G$ is sufficiently nice (see also [32, [14, 24]). 
Theorem 3.2.1. Suppose that $G$ has finite vcd and that $X$ is a discrete $G$-spectrum. Then there is an equivalence

$$
\begin{aligned}
X^{h G} & \simeq \underset{\Delta}{\operatorname{holim}} \Gamma_{G}^{\bullet} X \\
& =\mathbb{H}_{c}(G ; X),
\end{aligned}
$$

where $\mathbb{H}_{c}(G ; X)$ is the hypercohomology spectrum.

Proof. In [6, Thm. 7.4] it is proven that there is an equivalence

$$
X^{h G} \simeq \underset{\Delta}{\operatorname{holim}} \Gamma_{G}^{\bullet} X_{f G}
$$

(The cosimplicial object defining the hypercohomology spectrum is different, but isomorphic to that appearing in [6].) The result follows once we establish that the map induced from fibrant replacement

$$
\underset{\Delta}{\operatorname{holim}} \Gamma_{G}^{\bullet} X \rightarrow \underset{\Delta}{\operatorname{holim}} \Gamma_{G}^{\bullet} X_{f G}
$$

is an equivalence. This map is deduced to be an equivalence from the following facts: (a) the fibrant replacement map $X \rightarrow X_{f G}$ is an equivalence; (b) the functor $\Gamma_{G}$ preserves equivalences, by Lemma 2.4.1. (c) the homotopy limit construction sends levelwise equivalences to equivalences, since it is a Quillen derived functor.

3.3. Iterated homotopy fixed points. Let $U$ be an open subgroup of $G$, so that $G / U$ is finite.

Proposition 3.3.1. Let $X$ be a discrete $G$-spectrum.

(1) If $U$ is normal in $G$, the $U$-fixed point spectrum $\left(X_{f G}\right)^{U}$ is fibrant as a discrete $G / U$-spectrum.

(2) The fibrant discrete $G$-spectrum $X_{f G}$ is fibrant as a discrete $U$-spectrum.

(3) If $U$ is normal in $G$, the homotopy fixed point spectrum $X^{h U}$ is a $G / U$ spectrum.

(4) If $U$ is normal in $G$, there is an equivalence $X^{h G} \simeq\left(X^{h U}\right)^{h G / U}$.

Proof. To prove (1), observe that since $U$ is normal, for any discrete $G$-spectrum $Y$, the $U$-fixed point spectrum $Y^{U}$ is naturally a $G / U$-spectrum. There is an adjoint pair of functors $\left(\operatorname{Res}_{G / U}^{G},(-)^{U}\right)$

$$
\operatorname{Res}_{G / U}^{G}: \Sigma \operatorname{Sp}_{G / U} \rightleftarrows \Sigma \mathrm{Sp}_{G}:(-)^{U},
$$

where $\operatorname{Res}_{G / U}^{G}$ is defined by restriction along the quotient homomorphism $G \rightarrow$ $G / U$. Since $\operatorname{Res}_{G / U}^{G}$ preserves cofibrations and weak equivalences, the functor $(-)^{U}$ preserves fibrant objects.

We verify (2) in a similar way (compare with [26, Rmk. 6.26]). Define the induction functor on a discrete $U$-spectrum $Y$ to be

$$
\operatorname{Ind}_{U}^{G} Y=G_{+} \wedge_{U} Y .
$$

Here, $G_{+} \wedge_{U} Y$ is formed by regarding $G$ and $U$ as discrete groups, but this is easily seen to produce a discrete $G$-spectrum, since $U$ is a subgroup of finite index. The induction functor is the left adjoint of an adjunction

$$
\operatorname{Ind}_{U}^{G}: \Sigma \operatorname{Sp}_{U} \rightleftarrows \Sigma \operatorname{Sp}_{G}: \operatorname{Res}_{G}^{U},
$$


where $\operatorname{Res}_{G}^{U}$ is restriction along the inclusion $U \hookrightarrow G$. Since nonequivariantly there is an isomorphism

$$
\operatorname{Ind}_{U}^{G} Y \cong G / U_{+} \wedge Y
$$

we see that $\operatorname{Ind}_{U}^{G}$ preserves cofibrations and weak equivalences, from which it follows that $\operatorname{Res}_{G}^{U}$ preserves fibrant objects.

By (2), $X_{f G}$ is a fibrant discrete $U$-spectrum. Also, $X \rightarrow X_{f G}$ is a trivial cofibration of spectra and it is $U$-equivariant, so it is a trivial cofibration in $\Sigma \operatorname{Sp}_{U}$. Thus,

$$
X^{h U}=\left(X_{f G}\right)^{U}
$$

which is a $G / U$-spectrum. This proves (3).

(4) is proven using our fibrancy results. There are equivalences:

$$
X^{h G} \simeq X_{f G}^{G}=\left(X_{f G}^{U}\right)^{G / U} \simeq\left(X^{h U}\right)^{h G / U} .
$$

3.4. Homomorphisms of groups. If $f: H \rightarrow G$ is a continuous homomorphism of profinite groups, we may regard discrete $G$-sets as discrete $H$-sets. For a discrete $H$-set $Z$, we define the coinduced discrete $G$-set by

$$
f_{*} Z=\operatorname{CoInd}_{H}^{G} Z=\operatorname{Map}_{H}^{c}(G, Z)=\underset{U \unlhd_{o} G}{\operatorname{colim}} \operatorname{Map}_{H}(G / U, Z),
$$

where the $G$-action is defined by the formula

$$
(g \cdot \alpha)\left(g^{\prime} U\right)=\alpha\left(g^{\prime} g U\right),
$$

for $g \in G$ and $\alpha \in \operatorname{Map}_{H}(G / U, Z)$. This construction extends to simplicial discrete $G$-sets and discrete $G$-spectra in the obvious manner to give a functor

$$
f_{*}: \Sigma \mathrm{Sp}_{H} \rightarrow \Sigma \mathrm{Sp}_{G} .
$$

The functor $f_{*}$ is the right adjoint of an adjoint pair $\left(f^{*}, f_{*}\right)$, where

$$
f^{*}=\operatorname{Res}_{G}^{H}: \Sigma \operatorname{Sp}_{G} \rightarrow \Sigma \operatorname{Sp}_{H}
$$

is the restriction functor along the homomorphism $f$. Since $f^{*}$ clearly preserves cofibrations and weak equivalences, we have the following lemma.

Lemma 3.4.1. The adjoint functors $\left(f^{*}, f_{*}\right)$ form a Quillen pair. In particular, $f_{*}$ preserves fibrations and weak equivalences between fibrant objects.

We make the following observations.

(1) The Quillen pair $\left(f^{*}, f_{*}\right)$ gives rise to a derived adjoint pair $\left(L f^{*}, R f_{*}\right)$.

(2) Since the functor $f^{*}$ preserves all weak equivalences, there are equivalences $L f^{*} X \simeq f^{*} X$ for all discrete $G$-spectra $X$.

(3) If $j: H \hookrightarrow G$ is the inclusion of a closed subgroup, then for a discrete $H$-spectrum $X$, we have a nonequivariant isomorphism

$$
j_{*} X=\operatorname{Map}_{H}^{c}(G, X) \cong \operatorname{Map}^{c}(G / H, X) .
$$

By Lemma 2.4.1, we see that $j_{*}$ preserves weak equivalences, and therefore there is an equivalence $j_{*} X \simeq R j_{*} X$.

(4) The adjoint pair $\left(\operatorname{triv},(-)^{G}\right)$ of Section 3.1 agrees with the adjoint pair $\left(r^{*}, r_{*}\right)$ when $r: G \rightarrow\{e\}$ is the homomorphism to the trivial group. Therefore, the homotopy fixed point functor is given by $(-)^{h G}=R r_{*}$.

(5) Given continuous homomorphisms $H \stackrel{f}{\rightarrow} G \stackrel{g}{\rightarrow} K$, there are natural isomorphisms $(g \circ f)_{*} \cong g_{*} \circ f_{*}$ and $(g \circ f)^{*} \cong f^{*} \circ g^{*}$. We get similar formulas on the level of derived functors. 
(6) If $i: U \hookrightarrow G$ is the inclusion of an open subgroup, then the induction functor $i_{!}=\operatorname{Ind}_{U}^{G}$ (Proposition 3.3.1) is the left adjoint of the Quillen pair $\left(i_{!}, i^{*}\right)$.

We use these derived functors to prove a version of Shapiro's Lemma.

Lemma 3.4.2. Let $X$ be a discrete $G$-spectrum, and suppose that $H$ is a closed subgroup of $G$. Then there is an equivalence

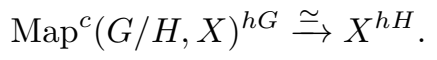

Proof. Consider the following diagram of groups:

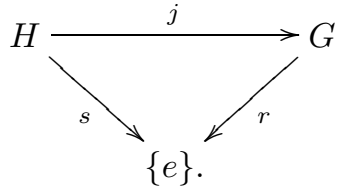

If $Z$ is a discrete $G$-set, there is a $G$-equivariant bijection

$$
\delta: j_{*} j^{*} Z=\operatorname{Map}_{H}^{c}(G, Z) \stackrel{\cong}{\longrightarrow} \operatorname{Map}^{c}(G / H, Z) .
$$

The map $\delta$ sends a map $\alpha$ in $\operatorname{Map}_{H}^{c}(G, Z)$ to the map

$$
\delta(\alpha): g H \mapsto g \alpha\left(g^{-1}\right) .
$$

The inverse $\delta^{-1}$ sends a map $\beta$ in $\operatorname{Map}^{c}(G / H, Z)$ to the map

$$
\delta^{-1}(\beta): g \mapsto g \beta\left(g^{-1} H\right)
$$

The isomorphism $\delta$ induces for a discrete $G$-spectrum $Y$ an isomorphism

$$
\delta: j_{*} j^{*} Y \stackrel{\cong}{\longrightarrow} \operatorname{Map}^{c}(G / H, Y)
$$

in $\Sigma \mathrm{Sp}_{G}$. By Lemma 3.4.1, the functor $j_{*}$ sends $H$-fibrant objects to $G$-fibrant objects. Therefore we have the equivalences:

$$
\begin{aligned}
\operatorname{Map}^{c}(G / H, X)^{h G} & \cong R r_{*} j_{*} j^{*} X \\
& \simeq R r_{*} R j_{*} j^{*} X \\
& \simeq R s_{*} j^{*} X \\
& =X^{h H} .
\end{aligned}
$$

3.5. Iterated fixed points for closed subgroups. We wish to extend the results of Section 3.3 to closed subgroups. The following proposition may be compared to [26, Lem. 6.35].

Proposition 3.5.1. Let $N$ be a closed normal subgroup of $G$, and let $X$ be a discrete $G$-spectrum. Then there is an equivalence

$$
\left(\left(X_{f G}\right)^{N}\right)^{h G / N} \simeq X^{h G} .
$$

Proof. Consider the following diagram:

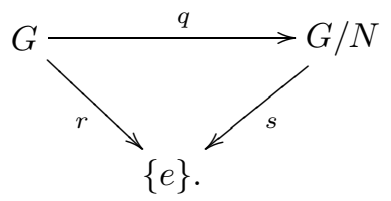


There is an equivalence

$$
\left(\left(X_{f G}\right)^{N}\right)^{h G / N}=R s_{*} R q_{*} X \simeq R r_{*} X=X^{h G} .
$$

Let $H$ be a closed subgroup of $G$. The reader might wonder if $X_{f G}$ is fibrant as a discrete $H$-spectrum, but this does not appear to hold for nontrivial closed subgroups $H$ that are not open. We discuss these difficulties in Section 3.6. Though $\left(X_{f G}\right)^{H}$ is not known to always equal $X^{h H}$, the following result identifies $\left(X_{f G}\right)^{H}$ with a canonical colimit that always maps to $X^{h H}$.

Corollary 3.5.2. Let $H$ be a closed subgroup of $G$. There is an equivalence

$$
\left(X_{f G}\right)^{H} \simeq \operatorname{colim}_{H \leq U \leq o} X^{h U} .
$$

Proof. Since $H$ acts discretely on $X_{f G}$, we have a canonical isomorphism

$$
\left(X_{f G}\right)^{H} \cong \operatorname{colim}_{H \leq U \leq o}\left(X_{f G}\right)^{U} .
$$

By Proposition 3.3.1 the spectrum $X_{f G}$ is fibrant as a discrete $U$-spectrum, so there are equivalences $\left(X_{f G}\right)^{U} \simeq X^{h U}$.

By Corollary 3.5.2, given a discrete $G$-spectrum $X$ and a closed subgroup $H$, there is a natural map

$$
\left(X_{f G}\right)^{H} \simeq \operatorname{colim}_{H \leq U \leq o} X^{h U} \rightarrow X^{h H} .
$$

If we restrict ourselves to the case where $G$ has finite cohomological dimension, then, as shown below, the iterated homotopy fixed point spectrum behaves in a more satisfactory way.

Proposition 3.5.3. Suppose that $G$ has finite cohomological dimension, and suppose that $X$ is a discrete $G$-spectrum. Let $H$ be a closed subgroup of $G$. Then the natural map

is an equivalence.

$$
\operatorname{colim}_{H \leq U \leq \leq_{o} G} X^{h U} \rightarrow X^{h H}
$$

Proof. For $K$ a profinite group of finite vcd, and $Y$ a discrete $K$-spectrum, let $E_{r}(K ; Y)$ denote the conditionally convergent descent spectral sequence

$$
E_{2}(K ; Y)=H_{c}^{*}\left(K ; \pi_{*}(Y)\right) \Rightarrow \pi_{*}\left(Y^{h K}\right) .
$$

There is a map of spectral sequences

$$
E_{r}^{\prime}(H ; X):=\operatorname{colim}_{H \leq U \leq o} E_{r}(U ; X) \rightarrow E_{r}(H ; X),
$$

which is an isomorphism on the level of $E_{2}$-terms by [42, Thm. 9.7.2]. The proposition now follows from [32, Prop. 3.3].

In Section 7.1, we will see that we may extend Proposition 3.5 .3 to groups of finite virtual cohomological dimension provided that we are taking homotopy fixed points of a consistent profaithful $k$-local profinite Galois extension. 
Corollary 3.5.4. Let $G$ be of finite cohomological dimension, and let $X$ be a discrete $G$-spectrum. Suppose that $H$ is a closed subgroup of $G$. Then there is an equivalence $\left(X_{f G}\right)^{H} \simeq X^{h H}$.

Theorem 3.5.5. Let $G$ be of finite cohomological dimension, and let $X$ be a fibrant discrete $G$-spectrum. Suppose that $H$ is a closed subgroup of $G$. Then $X$ is fibrant as a discrete $H$-spectrum.

Proof. By the proof of Theorem 2.3.2, the fibrant objects $Y$ of $\Sigma \mathrm{Sp}_{H}$ are precisely the objects for which the $Y_{i}$ are fibrant as simplicial discrete $H$-sets, and the maps

$$
Y_{i} \rightarrow \Omega Y_{i+1}
$$

are weak equivalences.

Since $X$ is fibrant as a discrete $G$-spectrum, each $X_{i}$ is fibrant as a simplicial discrete $G$-set, and the maps

$$
X_{i} \rightarrow \Omega X_{i+1}
$$

are weak equivalences. The only thing remaining to check is that each space $X_{i}$ is fibrant when regarded as a simplicial discrete $H$-set.

A criterion for fibrancy is established in [3, 10.2.7]. We remark that [3] deals with the more general class of locally compact totally disconnected groups $G$, acting on "simplicial smooth $G$-sets." However, in the case where $G$ is a profinite group, the category of smooth $G$-sets is the category of discrete $G$-sets. We need to check

(1) the $V$-fixed points $X_{i}^{V}$ are a Kan complex for every open subgroup $V \leq H$,

(2) for every open subgroup $V \leq H$ and every hypercover $\left\{H / V_{\alpha, \bullet}\right\}_{\alpha \in I_{\bullet}}$ of $H / V$, the induced map

$$
X_{i}^{V} \rightarrow \underset{\Delta}{\operatorname{holim}} \prod_{\alpha \in I} X_{i}^{V_{\alpha}, \bullet}
$$

is a weak equivalence.

Let $V$ be an open subgroup of $H$. We have

$$
X_{i}^{V}=\underset{V \leq U \leq o G}{\operatorname{colim}} X_{i}^{U} .
$$

Now $X_{i}^{U}$ is a Kan complex, since $X_{i}$ is fibrant as a simplicial discrete $G$-set. Since filtered colimits of Kan complexes are Kan complexes, we deduce that $X_{i}^{V}$ is a Kan complex. This verifies condition (1). We furthermore point out that for each open subgroup $U$ in $G$ containing $V$, Proposition 3.3.1 implies that the fixed point spectrum $X^{U}$ is fibrant. Therefore, the maps

$$
X_{i}^{U} \rightarrow \Omega X_{i+1}^{U}
$$

are weak equivalences. Since the functor $\Omega$ commutes with filtered colimits, and because filtered colimits of weak equivalences are weak equivalences, the maps

$$
X_{i}^{V} \rightarrow \Omega X_{i+1}^{V}
$$

are weak equivalences, and we deduce that the spectrum $X^{V}$ is fibrant (as a symmetric spectrum). By Proposition 3.3.1, we have

$$
\left(X_{f H}\right)^{V} \simeq X^{h V} .
$$

Corollary 3.5 .4 therefore implies that the map

$$
X^{V} \rightarrow\left(X_{f H}\right)^{V}
$$


is a weak equivalence. Since weak equivalences between fibrant symmetric spectra are levelwise equivalences, we deduce that each map

$$
X_{i}^{V} \rightarrow\left(X_{f H}\right)_{i}^{V}
$$

is a weak equivalence.

We now verify condition (2). Suppose that $\left\{H / V_{\alpha, \bullet}\right\}_{\alpha \in I_{\bullet}}$ is a hypercover of $H / V$. Consider the following diagram:

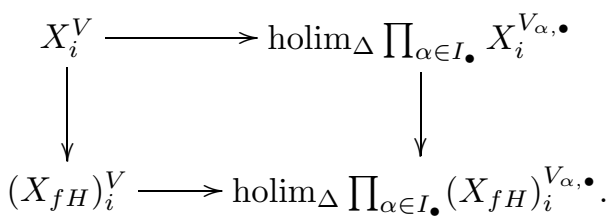

The bottom map is a weak equivalence since $X_{f H}$ is fibrant as a discrete $H$ spectrum. We have shown that the vertical maps are weak equivalences. We deduce that the top map is a weak equivalence.

Corollary 3.5.6. Let $X$ be a discrete $G$-spectrum. Suppose that $G$ has finite cohomological dimension, and suppose that $N$ is a closed normal subgroup of $G$. Then the homotopy fixed point spectrum $X^{h N}$ is a discrete $G / N$-spectrum, and there is an equivalence

$$
\left(X^{h N}\right)^{h G / N} \simeq X^{h G} .
$$

Proof. Since $X_{f G}$ is fibrant as a discrete $N$-spectrum, we have

$$
X^{h N}=\left(X_{f G}\right)^{N}
$$

and $\left(X_{f G}\right)^{N}$ is a discrete $G / N$-spectrum. Furthermore, by Proposition 3.5.1, we have

$$
X^{h G} \simeq\left(\left(X_{f G}\right)^{N}\right)^{h G / N} \simeq\left(X^{h N}\right)^{h G / N} .
$$

3.6. The difficulties concerning arbitrary closed fixed points. Let $H$ be a closed subgroup of an arbitrary profinite group $G$. We would be able to remove the finite cohomological dimension hypothesis in Section 3.5 if we knew that the restriction functor

$$
\operatorname{Res}_{G}^{H}: \Sigma \operatorname{Sp}_{G} \rightarrow \Sigma \operatorname{Sp}_{H}
$$

sends $G$-fibrant objects to $H$-fibrant objects. While we know of no counterexamples to this assertion, we also doubt that this is true in general.

We saw in Proposition 3.3.1 that for $U$ an open subgroup of $G$, the presence of an induction functor $\operatorname{Ind}_{U}^{G}$ which was a left Quillen adjoint to $\operatorname{Res}_{G}^{U}$ allowed us to prove that $\operatorname{Res}_{G}^{U}$ preserves fibrant objects.

However, as pointed out to the second author by Jeff Smith, $\operatorname{Res}_{G}^{H}$ cannot possess a left adjoint, in general, since it does not preserve limits. This can be seen as follows.

For a profinite group $K$ and a diagram $\left\{X_{\alpha}\right\}$ in the category $\Sigma \mathrm{Sp}_{K}$, let

$$
\lim _{\alpha}^{K} X_{\alpha}
$$

denote the limit computed in the category $\Sigma \mathrm{Sp}_{K}$. This limit is given by the following formula:

$$
\lim _{\alpha}{ }^{K} X_{\alpha}=\operatorname{colim}_{U \unlhd_{o} K}\left(\lim _{\alpha}{ }^{\mathrm{Sp}} X_{\alpha}\right)^{U} .
$$


Here, the limit $\lim ^{\mathrm{Sp}}$ is the limit computed in the underlying category of symmetric spectra.

Thus, given a diagram $\left\{X_{\alpha}\right\}$ in $\Sigma \mathrm{Sp}_{G}$, the restriction of the limit is given by

$$
\operatorname{Res}_{G}^{H} \lim _{\alpha}^{G} X_{\alpha}=\underset{U \unlhd_{o} G}{\operatorname{colim}}\left(\lim _{\alpha}^{\mathrm{Sp}} X_{\alpha}\right)^{U} .
$$

However, the limit of the restriction is computed to be

$$
\lim _{\alpha}^{H} \operatorname{Res}_{G}^{H} X_{\alpha}=\underset{V \unlhd_{o} H}{\operatorname{colim}}\left(\lim _{\alpha}{ }^{\mathrm{Sp}} X_{\alpha}\right)^{V} .
$$

When $H$ is not open in $G$, the lack of cofinality implies that these subspectra of $\lim _{\alpha}^{\mathrm{Sp}} X_{\alpha}$ in general do not agree.

One might suspect that one could still prove that the map

$$
\underset{H \leq U \leq \leq_{o} G}{\operatorname{colim}} X^{h U} \rightarrow X^{h H}
$$

is an equivalence if $G$ has finite virtual cohomological dimension, by a comparison of descent spectral sequences. This approach, however, also presents difficulties. As in the proof of Proposition 3.5.3, there is a map of spectral sequences

$$
E_{r}^{\prime}(H ; X)=\operatorname{colim}_{H \leq U \leq o G} E_{r}(U ; X) \rightarrow E_{r}(H ; X),
$$

which is an isomorphism on the level of $E_{2}$-terms (see [42, Thm. 9.7.2]). The problem is that the colimit of the spectral sequences does not converge to the colimit of the abutments in general.

\section{Continuous $G$-SPectra}

In this paper, a continuous $G$-spectrum is a pro-object in the category of discrete $G$-spectra. In this section, we extend some of our constructions for $\Sigma \operatorname{Sp}_{G}$ to the category of continuous $G$-spectra. For continuous $G$-spectra that are indexed over $\{0 \leftarrow 1 \leftarrow 2 \leftarrow \cdots\}$, part of this material appears in more detail in [6].

4.1. Pro-objects in discrete $G$-spectra. Following standard usage, a pro-object in a category $\mathcal{C}$ is a cofiltered diagram in $\mathcal{C}$. We define the category of continuous $G$-spectra $\Sigma \operatorname{Sp}_{G}^{c}$ to be the category of pro-objects in $\Sigma \mathrm{Sp}_{G}$. Thus, a continuous $G$-spectrum is a cofiltered diagram $\mathbf{X}=\left\{X_{i}\right\}_{i \in I}$ of discrete $G$-spectra. Maps in the category of continuous $G$-spectra are given by

$$
\Sigma \operatorname{Sp}_{G}^{c}(\mathbf{X}, \mathbf{Y})=\lim _{j} \operatorname{colim}_{i} \Sigma \operatorname{Sp}_{G}\left(X_{i}, Y_{j}\right) .
$$

Any pro-spectrum $\mathbf{X}=\left\{X_{i}\right\}$ gives rise to a spectrum $X$ via the homotopy limit functor:

$$
X=\operatorname{holim}_{i} X_{i} .
$$

We shall always denote our pro-spectra by boldface type and their homotopy limits with nonboldface type.

Remark 4.1.1. A more general theory of pro-spectra, including a model category structure, has been developed by Isaksen (see [23, and [13, Section 1.1]). Fausk has developed a category of continuous genuine $G$-spectra where $G$ is a compact Hausdorff topological group [12. The notion of a continuous $G$-spectrum in this paper (that is, a pro-discrete $G$-spectrum) corresponds roughly, in [12, to a pro$G$-spectrum that is in the full subcategory of cofibrant objects in the Postnikov 
$\overline{\operatorname{Lie}(G)}$-model structure on pro- $\mathcal{M}_{S}$. For more details, we refer the reader to [12, Section 11.3], especially the discussion centered around [op. cit., Eq. (11-15)].

4.2. Continuous mapping spectra. Let $K=\lim _{i} K_{i}$ be a profinite $G$-set. Given a continuous $G$-spectrum $\mathbf{X}$, the continuous mapping spectrum $\operatorname{Map}^{c}(K, \mathbf{X})$ is defined to be the continuous $G$-spectrum

$$
\left\{\operatorname{Map}^{c}\left(K, X_{j}\right)\right\}_{j} \text {. }
$$

We denote the homotopy limit of $\operatorname{Map}^{c}(K, \mathbf{X})$ by $\operatorname{Map}^{c}(K, \mathbf{X})$. If $K$ satisfies the hypotheses of Lemma 2.4.1, and the derived functors $\lim _{j}^{s} \operatorname{Map}^{c}\left(K, \pi_{t}\left(X_{j}\right)\right)=0$, for all $s>0$ and all $t \in \mathbb{Z}$, then the Bousfield-Kan spectral sequence

$$
\lim _{j}^{s} \operatorname{Map}^{c}\left(K, \pi_{t}\left(X_{j}\right)\right) \Rightarrow \pi_{*}\left(\operatorname{Map}^{c}(K, \mathbf{X})\right)
$$

collapses, and thus,

$$
\pi_{*}\left(\operatorname{Map}^{c}(K, \mathbf{X})\right) \cong \operatorname{Map}^{c}\left(K, \lim _{j} \pi_{*}\left(X_{j}\right)\right) .
$$

4.3. Continuous permutation spectra. Let $K=\lim _{i} K_{i}$ be a profinite $G$-set, and let each finite set $K_{j}$, for each $j$ in the indexing set for $K$, be a discrete $G$-set. Also, let $\mathbf{X}=\left\{X_{i}\right\}_{i}$ be a continuous $G$-spectrum. Define the permutation spectrum $\mathbf{X}[[K]]$ to be the continuous $G$-spectrum given by

$$
\left\{X_{i}\left[K_{j}\right]\right\}_{i, j} .
$$

We denote the homotopy limit of $\mathbf{X}[[K]]$ by

$$
X[[K]]=\operatorname{holim}_{i, j} X_{i}\left[K_{j}\right] .
$$

Note that if $\lim _{i, j}^{s} \pi_{t}\left(X_{i}\right)\left[K_{j}\right]=0$, for all $s>0$ and all $t \in \mathbb{Z}$ (where $\pi_{t}\left(X_{i}\right)\left[K_{j}\right]$ is an abelian group), then

$$
\pi_{*}(X[[K]]) \cong \lim _{i, j} \pi_{*}\left(X_{i}\right)\left[K_{j}\right] .
$$

If $E$ is a discrete $G$-spectrum, we use $\mathbf{E}[[K]]$ to denote the continuous $G$-spectrum $\left\{E\left[K_{j}\right]\right\}_{j}$.

4.4. Continuous homotopy fixed points. For a continuous $G$-spectrum $\mathbf{X}$, we define the homotopy fixed point pro-spectrum $\mathbf{X}^{h G}$ to be

$$
\left\{X_{i}^{h G}\right\}_{i} \text {. }
$$

We denote the homotopy limit of $\mathbf{X}^{h G}$ by $X^{h G}$, and we refer to $X^{h G}$ as the homotopy fixed point spectrum.

4.5. Continuous hypercohomology spectra. We define

$$
\boldsymbol{\Gamma}_{G}:(\text { pro }-\Sigma \mathrm{Sp}) \rightarrow(\text { pro }-\Sigma \mathrm{Sp})
$$

to be the coaugmented comonad given by

$$
\boldsymbol{\Gamma}_{G}(\mathbf{X})=\operatorname{Map}^{c}(G, \mathbf{X}) .
$$

Let $\Gamma_{G}(\mathbf{X})$ be the homotopy limit of $\boldsymbol{\Gamma}_{G}(\mathbf{X})$.

If $\mathbf{X}$ is a continuous $G$-spectrum, then it is a coalgebra over $\boldsymbol{\Gamma}_{G}$. Let $\mathbb{H}_{c}(G ; \mathbf{X})$ denote the pro-spectrum obtained by taking hypercohomology levelwise:

$$
\mathbb{H}_{c}(G ; \mathbf{X})=\left\{\mathbb{H}_{c}\left(G ; X_{i}\right)\right\}_{i} .
$$

Let $\mathbb{H}_{c}(G ; X)$ denote the homotopy limit of the pro-spectrum $\mathbb{H}_{c}(G ; \mathbf{X})$. The following result follows immediately from Theorem 3.2 .1 
Theorem 4.5.1. Suppose that $\mathbf{X}=\left\{X_{i}\right\}$ is a continuous $G$-spectrum. If $G$ has finite vcd, then there is an equivalence

$$
X^{h G} \simeq \mathbb{H}_{c}(G ; X) .
$$

4.6. Homotopy fixed point spectral sequence. Let $G$ have finite vcd. Then Theorem 4.5.1 implies that

$$
X^{h G} \simeq \underset{\Delta}{\operatorname{holim}} \operatorname{holim} \Gamma_{G}^{\bullet} X_{i},
$$

and, hence, the associated Bousfield-Kan spectral sequence has the form

$$
E_{2}^{s, t}(G ; X)=\pi^{s} \pi_{t}\left(\underset{i}{\operatorname{holim}} \Gamma_{G}^{\bullet} X_{i}\right) \Rightarrow \pi_{t-s}\left(X^{h G}\right),
$$

giving the conditionally convergent homotopy fixed point spectral sequence for $X^{h G}$.

Observe that there is a natural isomorphism

$$
\Gamma_{G}^{k}(-) \cong \operatorname{Map}^{c}\left(G^{k},-\right) .
$$

If $\lim _{i}^{s} \operatorname{Map}^{c}\left(G^{k}, \pi_{t}\left(X_{i}\right)\right)=0$ for all $s>0$, all $k \geq 0$, and all $t \in \mathbb{Z}$, then for each $k \geq 0$, the Bousfield-Kan spectral sequence

$$
\lim _{i}^{s} \operatorname{Map}^{c}\left(G^{k}, \pi_{t}\left(X_{i}\right)\right) \Rightarrow \pi_{*}\left(\operatorname{holim}_{i} \operatorname{Map}^{c}\left(G^{k}, X_{i}\right)\right)
$$

collapses, and thus,

$$
\begin{aligned}
E_{2}^{s, t}(G ; X) & \cong \pi^{s}\left(\lim _{i} \operatorname{Map}^{c}\left(G^{\bullet}, \pi_{t}\left(X_{i}\right)\right)\right) \\
& \cong H^{s}\left(\operatorname{Map}^{c}\left(G^{\bullet}, \lim _{i} \pi_{t}\left(X_{i}\right)\right)\right) \\
& \cong H^{s}\left(\operatorname{Map}^{c}\left(G \bullet, \pi_{t}(X)\right)\right) \\
& \cong H_{c}^{s}\left(G ; \pi_{t}(X)\right) .
\end{aligned}
$$

Here, $H_{c}^{s}\left(G ; \pi_{t}(X)\right)$ denotes the continuous cohomology of continuous cochains, with coefficients in the topological $G$-module $\pi_{t}(X) \cong \lim _{i} \pi_{t}\left(X_{i}\right)$.

4.7. Completed smash product. If $\mathbf{X}$ and $\mathbf{Y}$ are continuous $G$-spectra, we define the completed smash product $\mathbf{X} \wedge_{c} \mathbf{Y}$ to be the continuous $G$-spectrum

$$
\left\{X_{i} \wedge Y_{j}\right\}_{i, j}
$$

The completed smash product gives $\Sigma \mathrm{Sp}_{G}^{c}$ a symmetric monoidal product, where the unit is $\left\{S^{0}\right\}$ (the sphere spectrum regarded as a diagram indexed by a single element).

\section{Modules And Commutative algebras of Discrete $G$-SPeCtra}

Let $A$ be a commutative symmetric ring spectrum and let $G$ be a profinite group. In this section, we describe the model categories of discrete $G$ - $A$-modules and discrete commutative $G$ - $A$-algebras. We show that the homotopy fixed points of a discrete $G$ - $A$-module are an $A$-module and the homotopy fixed points of a discrete commutative $G$ - $A$-algebra are a commutative $A$-algebra. These structured homotopy fixed point constructions are shown to agree, in the stable homotopy category, with the usual homotopy fixed points of the underlying discrete $G$-spectrum. We then make comparisons between filtered homotopy colimits and filtered colimits of modules and commutative algebras and conclude that, when properly interpreted, they all coincide in the stable homotopy category. We conclude this section by 
describing how to make the hypercohomology spectra of discrete commutative $G$ $A$-algebras take values in the category of commutative $A$-algebras.

5.1. Modules of discrete $G$-spectra. Let $A$ be a commutative symmetric ring spectrum. By a discrete $G$ - $A$-module, we shall mean a discrete $G$-spectrum $X$ that also possesses the structure of an $A$-module. We require these structures to be compatible in the following sense: for every element $g \in G$, the following diagram must commute:

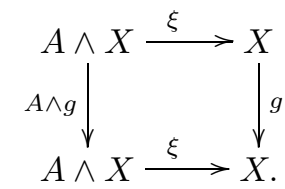

Here, $\xi$ is the $A$-module structure map. Let $\operatorname{Mod}_{G, A}$ denote the category of discrete $G$ - $A$-modules, with morphisms being the $G$-equivariant maps that are also maps of $A$-modules. Note that, given discrete $G$ - $A$-modules $X$ and $Y$, their smash product $X \wedge_{A} Y$ is easily seen to be a discrete $G$ - $A$-module with the diagonal action.

The following simplified variant of D.M. Kan's "lifting theorem" will be used repeatedly to provide the desired model structures on structured categories such as $\operatorname{Mod}_{G, A}$.

Lemma 5.1.1. Suppose that $\mathcal{M}$ is a cofibrantly generated model category with generating cofibrations I and generating trivial cofibrations $J$. Furthermore, assume that the domains of $I$ and $J$ are $\alpha$-small for some cardinal $\alpha$. Suppose that we are given a complete and cocomplete category $\mathcal{N}$ and an adjoint pair $(F, G)$,

$$
F: \mathcal{M} \rightleftarrows \mathcal{N}: G,
$$

where:

(1) G commutes with filtered colimits, and

(2) G takes relative FJ-cell complexes to weak equivalences.

Then $\mathcal{N}$ admits an induced model category structure, where the fibrations and weak equivalences are those morphisms which get sent to fibrations and weak equivalences by $G$, and the cofibrations are determined. This model category structure is cofibrantly generated with generating cofibrations FI and generating trivial cofibrations $F J$. The domains of FI and FJ are $\alpha$-small in $\mathcal{N}$.

Proof. This lemma is a special case of Theorem 11.3.2 of [17. To see that the hypotheses of this theorem are met in our situation, we must verify that the domains of $F I$ and $F J$ are $\alpha$-small with respect to relative $F I$ - and $F J$-cell complexes, respectively. However, our hypotheses imply that $F$ preserves all $\alpha$-small objects: given an $\alpha$-small object $X \in \mathcal{M}$, and a $\lambda$-sequence $(\lambda \geq \alpha)$

$$
Y_{1} \rightarrow Y_{2} \rightarrow Y_{3} \rightarrow \cdots \rightarrow Y_{\beta} \rightarrow \cdots \quad(\beta<\lambda)
$$

in $\mathcal{N}$, we have:

$$
\begin{aligned}
\operatorname{colim}_{i} \operatorname{Hom}_{\mathcal{N}}\left(F X, Y_{i}\right) & \cong \operatorname{colim}_{i} \operatorname{Hom}_{\mathcal{M}}\left(X, G Y_{i}\right) \\
& \cong \operatorname{Hom}_{\mathcal{M}}\left(X, \operatorname{colim}_{i} G Y_{i}\right) \\
& \cong \operatorname{Hom}_{\mathcal{M}}\left(X, G \operatorname{colim} Y_{i}\right) \\
& \cong \operatorname{Hom}_{\mathcal{N}}\left(F X, \operatorname{colim}_{i} Y_{i}\right) .
\end{aligned}
$$


In [29], a model category structure is defined on the category of $A$-modules. The fibrations and weak equivalences of this model structure are the fibrations and weak equivalences of underlying symmetric spectra, and the cofibrations are determined.

Proposition 5.1.2. The category $\operatorname{Mod}_{G, A}$ is a model category, where the fibrations and weak equivalences are the fibrations and weak equivalences of the underlying discrete G-spectra, and the cofibrations are the cofibrations of the underlying $A$ modules.

Proof. We apply Lemma 5.1.1 to the adjoint pair

$$
A \wedge-: \Sigma \operatorname{Sp}_{G} \rightleftarrows \operatorname{Mod}_{G, A}: \mathcal{U},
$$

where $\mathcal{U}$ is the forgetful functor.

The category $\Sigma \mathrm{Sp}_{G}$ is cofibrantly generated with generating cofibrations

$$
I=\left\{F_{n}\left(G / U \times \partial \Delta^{n}\right)_{+} \hookrightarrow F_{n}\left(G / U \times \Delta^{n}\right)_{+}: U \leq_{o} G, n \geq 0\right\} .
$$

(Here, $F_{n}$ is the left adjoint to the functor which returns the $n$th space of a symmetric spectrum.) Thus, the domains of the maps in $I$ are $\omega$-small. Since the model structure on $\Sigma \mathrm{Sp}_{G}$ is left proper and cellular, the Bousfield-Smith cardinality argument [17, Prop. 4.5.1] implies that there exists a cardinal $\alpha$, such that the collection $J$ of inclusions of $I$-cell complexes of size at most $\alpha$, which are weak equivalences, generates the trivial cofibrations of $\Sigma \mathrm{Sp}_{G}$. In particular, since the $I$-cells are finite, the domains of $J$ are $\alpha$-small.

By Lemma 5.3.4 below, the functor $\mathcal{U}$ preserves filtered colimits. We need to verify that $\mathcal{U}$ takes relative $(A \wedge J)$-cell complexes to weak equivalences. However, the domains and codomains of the maps in $J$ are cofibrant objects in $\Sigma$ Sp, and $A \wedge-$ preserves stable equivalences between cofibrant symmetric spectra.

The model structure on $\operatorname{Mod}_{G, A}$ given by Lemma 5.1.1 has the desired fibrations and weak equivalences. We need to check that the cofibrations of this model structure are precisely the cofibrations of underlying nonequivariant $A$-modules. The cofibrations are generated by $A \wedge I$. Since all of the morphisms in $I$ are cofibrations of underlying nonequivariant symmetric spectra, we deduce that all of the cofibrations of $G$ - $A$-modules are cofibrations of underlying $A$-modules. The converse is an argument similar to Corollary 1.10 of [14].

Given a discrete $G$ - $A$-module $X$, let $X_{f G A}$ denote a functorial fibrant replacement in $\operatorname{Mod}_{G, A}$, so that the fibrant replacement map

$$
X \rightarrow X_{f G A}
$$

is a trivial cofibration of discrete $G$ - $A$-modules. We define the homotopy fixed point $A$-module to be the fixed point spectrum

$$
X^{h_{A} G}=\left(X_{f G A}\right)^{G}
$$

The properties of our model category structures immediately give the following lemma.

Lemma 5.1.3. The spectrum $X_{f G A}$ is fibrant as a discrete $G$-spectrum, and there exists a weak equivalence

$$
X_{f G} \stackrel{\simeq}{\rightarrow} X_{f G A}
$$

in the category $\Sigma^{\Sigma} \mathrm{Sp}_{G}$. 
Since $(-)^{G}$ preserves weak equivalences between fibrant spectra in $\Sigma \mathrm{Sp}_{G}$, we have the following corollary.

Corollary 5.1.4. There is an equivalence

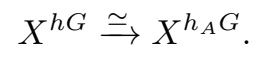

Since colimits of discrete $G$ - $A$-modules are formed on the level of underlying symmetric spectra, the comonad $\Gamma_{G}(-)=\operatorname{Map}^{c}(G,-)$ restricts to the category of $A$-modules, where, given an $A$-module $X$, the $A$-module structure is given by the composition

$$
A \wedge \operatorname{Map}^{c}(G, X) \rightarrow \operatorname{Map}^{c}(G, A \wedge X) \stackrel{\xi_{*}}{\rightarrow} \operatorname{Map}^{c}(G, X) .
$$

In this composition, the first map is given by the composite

$$
\begin{aligned}
A \wedge \operatorname{Map}^{c}(G, X) & =A \wedge \operatorname{colim}_{N \unlhd_{o} G} \operatorname{Map}(G / N, X) \\
& \cong \operatorname{colim}_{N \unlhd_{o} G} A \wedge \operatorname{Map}(G / N, X) \\
& \rightarrow \operatorname{colim}_{N \unlhd_{o} G} \operatorname{Map}(G / N, A \wedge X) \\
& =\operatorname{Map}^{c}(G, A \wedge X) .
\end{aligned}
$$

Since fibrant discrete $G$ - $A$-modules are fibrant in $\Sigma \mathrm{Sp}_{G}$, the following proposition is an immediate consequence of Theorem 3.2.1.

Proposition 5.1.5. Suppose $G$ has finite vcd, and let $X$ be a discrete $G$-A-module. Then there is an equivalence of $A$-modules

$$
X^{h_{A} G} \simeq \underset{\Delta}{\operatorname{holim}_{G}} \Gamma_{G}^{\bullet} X=\mathbb{H}_{c}(G ; X)
$$

Define the category $\operatorname{Mod}_{G, A}^{c}$ of continuous $G$-A-modules to be the category of pro-objects in $\operatorname{Mod}_{G, A}$. Given a continuous $G$ - $A$-module $\mathbf{X}=\left\{X_{i}\right\}_{i}$, we define

$$
\begin{aligned}
X^{h_{A} G} & :=\operatorname{holim}_{i} X_{i}^{h_{A} G}, \\
\mathbb{H}_{c}(G ; X) & :=\operatorname{holim}_{i} \mathbb{H}_{c}\left(G ; X_{i}\right) .
\end{aligned}
$$

Proposition 5.1.5 has the following corollary.

Corollary 5.1.6. Suppose $G$ has finite vcd, and let $\mathbf{X}$ be a continuous $G$-A-module. Then there is an equivalence of $A$-modules

$$
X^{h_{A} G} \simeq \mathbb{H}_{c}(G ; X)
$$

We shall henceforth drop the distinction between $(-)^{h_{A} G}$ and $(-)^{h G}$ : all homotopy fixed points of discrete $G$ - $A$-modules will implicitly be taken in the category of discrete $G$ - $A$-modules.

5.2. Commutative algebras of discrete $G$-spectra. By a discrete commutative $G$-A-algebra, we shall mean a discrete $G$ - $A$-module $E$ together with a commutative $A$-algebra multiplication

$$
\mu: E \wedge_{A} E \rightarrow E
$$

such that $G$ acts on $E$ through maps of commutative $A$-algebras. Let $\operatorname{Alg}_{A, G}$ denote the category of discrete commutative $G$-A-algebras, with morphisms being those morphisms in $\operatorname{Mod}_{G, A}$ that are also maps of commutative $A$-algebras. Following 
29], to place a model structure on $\operatorname{Alg}_{A, G}$, we need to replace the model structure on $\Sigma \mathrm{Sp}_{G}$ with a Quillen equivalent "positive" model structure.

Lemma 5.2.1. The category of discrete G-spectra admits a positive stable model structure, which we denote by $\Sigma \mathrm{Sp}_{G}^{+}$, where the cofibrations are the positive cofibrations of underlying symmetric spectra, the weak equivalences are the stable equivalences of underlying symmetric spectra, and the positive fibrations are determined.

Proof. We follow [29, Sec. 14], in its construction of the positive stable model structure on symmetric spectra, from start to finish, with some mild alterations. The category $\Sigma \mathrm{Sp}_{G}$ admits a positive level model structure that is defined as follows:

- the positive level fibrations are those maps $f: X \rightarrow Y$ where the maps $f_{i}: X_{i} \rightarrow Y_{i}$ are fibrations of simplicial discrete $G$-sets for $i \geq 1$;

- the positive level weak equivalences are those maps $f: X \rightarrow Y$ where the maps $f_{i}: X_{i} \rightarrow Y_{i}$ are weak equivalences of underlying simplicial sets, for all $i \geq 1$; and

- the positive cofibrations are those morphisms $X \rightarrow Y$ where the induced map

$$
X_{0} \rightarrow Y_{0}
$$

is an isomorphism and each of the induced maps

$$
\begin{aligned}
X_{i} & \rightarrow Y_{i}, & i=1, \\
X_{i} \cup_{L_{i} X} L_{i} Y & \rightarrow Y_{i}, & i \geq 2,
\end{aligned}
$$

is a projective cofibration in $\left(s \operatorname{Set}_{G}\right)_{*}^{\Sigma_{i}}$, where $L_{i}$ is the latching object of [20, Def. 8.4] (that is to say, they are precisely the positive cofibrations of underlying symmetric spectra).

The positive level model structure on $\Sigma \mathrm{Sp}_{G}$ is left proper and cellular, and hence, it admits a localization with respect to the set of maps

$$
F_{n} S^{1} \wedge C \rightarrow F_{n+1} C, \quad n \geq 1,
$$

where, as usual, $F_{n}$ is the left adjoint to the $n$th space evaluation functor and $C$ runs through the cofibrant domains and codomains of the generating cofibrations of $s \operatorname{Set}_{G}$. This localized model structure is the positive stable model structure:

- the cofibrations are those morphisms which are positive cofibrations on the underlying symmetric spectra;

- the fibrant objects are the discrete $G$-spectra $X$ for which

(1) the spaces $X_{i}$ are fibrant as simplicial discrete $G$-sets for $i \geq 1$,

(2) the spectrum structure maps $X_{i} \rightarrow \Omega X_{i+1}$ are weak equivalences for $i \geq 1$;

- the weak equivalences between fibrant objects are those morphisms that are positive level weak equivalences of discrete $G$-spectra.

We are left with verifying that the weak equivalences of the positive stable model structure are precisely the stable equivalences of underlying symmetric spectra. It suffices to check this for morphisms between positive stable fibrant objects. Let $\phi: X \rightarrow Y$ be a morphism between positive stable fibrant objects in $\Sigma \mathrm{Sp}_{G}$, and 
consider the functorial fibrant replacement (in the nonpositive stable model structure) of $\phi$ in $\Sigma \mathrm{Sp}_{G}$ :

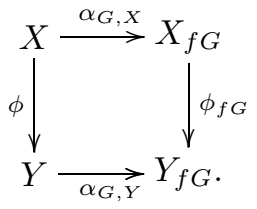

Note that the stably fibrant objects of $\Sigma \mathrm{Sp}_{G}$ are positive stable fibrant. The two morphisms $\alpha_{G,-}$ are therefore positive stable equivalences between positive stable fibrant discrete $G$-spectra, and therefore are positive level weak equivalences. It follows that they induce isomorphisms on naïve homotopy groups and hence are stable equivalences [21, Thm. 3.1.11]. The same argument shows that if $\phi$ is a positive stable equivalence, then $\phi$ is a stable equivalence. Conversely, suppose that $\phi$ is a stable equivalence. Then, by the two out of three axiom, the morphism $\phi_{f G}$ is a stable equivalence. Since $\phi_{f G}$ is a stable equivalence between stable fibrant discrete $G$-spectra, we deduce that it is a levelwise equivalence. In particular, it is a positive level weak equivalence. This allows us to deduce that $\phi$ is a positive level weak equivalence, and thus, it is a positive stable equivalence.

Lemma 5.2.2. The identity functor from discrete $G$-spectra with the positive stable model structure to discrete $G$-spectra with the stable model structure is the left adjoint of a Quillen equivalence.

Proof. This follows from the fact that the weak equivalences in both model structures are the same, and every positive cofibration is a cofibration.

The category $\mathrm{Alg}_{S}$ of commutative symmetric ring spectra admits a model category structure where the weak equivalences and fibrations are detected on the level of underlying symmetric spectra (with the positive model structure); see [29]. The category $\operatorname{Alg}_{A}$ may be regarded as the category of commutative symmetric ring spectra under $A$ and hence inherits a model structure with the same cofibrations, fibrations, and weak equivalences.

Theorem 5.2.3. The category of discrete commutative $G$-A-algebras admits a positive model structure, where the cofibrations are the cofibrations of underlying commutative symmetric ring spectra, the weak equivalences are the stable equivalences of underlying symmetric spectra, and the fibrations are the positive fibrations of discrete $G$-spectra.

Proof. The proof follows 29, Thm. 15.2(i)]. The category of discrete commutative $G$ - $A$-algebras is the undercategory $\left(A \downarrow \operatorname{Alg}_{G}\right)$, where $A$ is regarded as a discrete $G$ spectrum with the trivial action and $\operatorname{Alg}_{G}$ denotes the category $\operatorname{Alg}_{S, G}$ of discrete commutative $G$-algebras. Therefore, $\mathrm{Alg}_{A, G}$ inherits a model structure from $\mathrm{Alg}_{G}$, by [17, Thm. 7.6.5(1)]. Hence, it suffices to prove the theorem when $A=S$. We apply Lemma 5.1.1 to the adjoint pair

$$
\mathbb{P}: \Sigma \mathrm{Sp}_{G}^{+} \rightleftarrows \operatorname{Alg}_{G}: \mathbb{U},
$$

where $\mathbb{U}$ is the forgetful functor, and $\mathbb{P}$ is the free commutative algebra functor:

$$
\mathbb{P}(X)=\bigvee_{i \geq 0}\left(X^{\wedge i}\right)_{\Sigma_{i}}
$$


The category $\Sigma \mathrm{Sp}_{G}^{+}$is cofibrantly generated with generating cofibrations

$$
I^{+}=\left\{F_{n}\left(G / U \times \partial \Delta^{n}\right)_{+} \hookrightarrow F_{n}\left(G / U \times \Delta^{n}\right)_{+}: U \leq_{o} G, n \geq 1\right\} .
$$

The domains of the maps in $I^{+}$are thus $\omega$-small. The same Bousfield-Smith cardinality argument used in the proof of Proposition 5.1.2 shows that there exists a cardinal $\alpha$ and a set $J^{+}$of generating trivial cofibrations such that the domains of $J^{+}$are $\alpha$-small.

By Lemma 5.3 .4 below, the functor $\mathbb{U}$ preserves filtered colimits. We just need to verify that for any stable equivalence

$$
\phi: X \rightarrow Y
$$

the induced morphism

$$
\mathbb{P}(\phi): \mathbb{P}(X) \rightarrow \mathbb{P}(Y)
$$

is a stable equivalence. It suffices to verify that the map on coinvariants

$$
\phi_{i}:\left(X^{\wedge i}\right)_{\Sigma_{i}} \rightarrow\left(Y^{\wedge i}\right)_{\Sigma_{i}}
$$

is a stable equivalence for every $i$. Consider the following diagram of canonical maps:

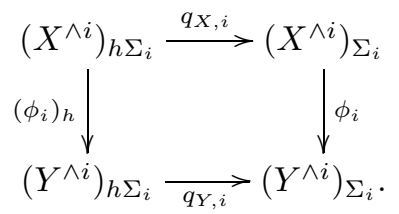

Since homotopy colimits preserve weak equivalences, we deduce that the maps $\left(\phi_{i}\right)_{h}$ are stable equivalences. The morphisms $q_{-, i}$ induce isomorphisms of naïve homotopy groups: this is seen by applying the geometric realization functor levelwise and by using Lemma 15.5 of 29] (the geometric realization of a positive cofibrant symmetric spectrum of simplicial sets is easily seen to be a positive cofibrant symmetric spectrum of topological spaces). Therefore, by [21, Thm. 3.1.11], the morphisms $q_{-, i}$ are stable equivalences. We deduce that each $\phi_{i}$ is a stable equivalence, as desired.

We have the following corollary.

Corollary 5.2.4. The following pairs of adjoint functors are Quillen adjoints:

$$
\begin{aligned}
& \text { triv: } \Sigma \mathrm{Sp}^{+} \rightleftarrows \Sigma \operatorname{Sp}_{G}^{+}:(-)^{G}, \\
& \text { triv: } \operatorname{Alg}_{A} \rightleftarrows \operatorname{Alg}_{A, G}:(-)^{G} .
\end{aligned}
$$

For a discrete $G$-spectrum $X$, we use $X_{f G^{+}}$to denote the functorial fibrant replacement in the positive model structure, and we denote the corresponding homotopy fixed point spectrum by

$$
X^{h^{+} G}=\left(X_{f^{+} G}\right)^{G}
$$

We have the following result.

Lemma 5.2.5. If $X$ is a discrete $G$-spectrum, then there is an equivalence

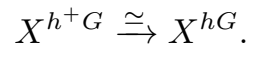


Proof. Since fibrant discrete $G$-spectra are positive fibrant discrete $G$-spectra, there is a stable equivalence

$$
\alpha: X_{f+G} \rightarrow X_{f G}
$$

in the category $\Sigma \operatorname{Sp}_{G}$. Since $(-)^{G}$ preserves stable equivalences between positive fibrant discrete $G$-spectra, $\alpha$ induces an equivalence

$$
X^{h^{+} G}=\left(X_{f+G}\right)^{G} \stackrel{\alpha_{*}}{\longrightarrow}\left(X_{f G}\right)^{G}=X^{h G} .
$$

Given a discrete commutative $G$-A-algebra $E$, we shall denote the functorial fibrant replacement by $E_{f G A-A l g}$. We define the homotopy fixed point commutative $A$-algebra to be the fixed point spectrum

$$
E^{h_{\mathrm{Alg}} G}=\left(E_{f G A-\mathrm{Alg}}\right)^{G} .
$$

Since $E_{f G A-\mathrm{Alg}}$ is a positive fibrant discrete $G$-spectrum, a slight modification of the proof of Lemma 5.2 .5 implies the following lemma.

Lemma 5.2.6. If $E$ is a discrete commutative $G$-A-algebra, then there is an equivalence

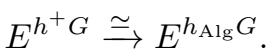

If $E$ is a discrete commutative $G$-A-algebra, then Corollary [5.1.4 and Lemmas 5.2.5 and 5.2.6 imply that there is the following zig-zag of equivalences:

$$
E^{h_{A} G} \stackrel{\simeq}{\simeq} E^{h G} \stackrel{\simeq}{\simeq} E^{h^{+} G} \stackrel{\simeq}{\rightrightarrows} E^{h_{\mathrm{Alg}} G} .
$$

Therefore, we shall henceforth not distinguish between the four equivalent types of homotopy fixed points that appear in the above zig-zag. Also, homotopy fixed points of discrete commutative $G$ - $A$-algebras will always implicitly be taken in the category of discrete commutative $G$ - $A$-algebras.

5.3. Filtered colimits. We will make frequent use of filtered colimits. In this section, we show that filtered colimits of spectra are rather well-behaved. We begin with the following lemma, whose proof follows the proofs of [41, Lemma 5.5] and [32, Proposition 3.2] (written in the context of Bousfield-Friedlander spectra). We remind the reader that a fibrant spectrum is positive fibrant.

Lemma 5.3.1. In the category of symmetric spectra, filtered colimits preserve cofibrations, fibrations, and positive fibrations. Filtered colimits preserve weak equivalences between fibrant spectra, and weak equivalences between positive fibrant spectra.

Corollary 5.3.2. Given a filtered diagram $\left\{X_{\alpha}\right\}_{\alpha \in I}$ of (positive) fibrant spectra, there is a stable equivalence

$$
\underset{\alpha}{\operatorname{hocolim}} X_{\alpha} \stackrel{\simeq}{\rightarrow} \operatorname{colim}_{\alpha} X_{\alpha} \text {. }
$$

Proof. Let $\left\{\widetilde{X}_{\alpha}\right\} \stackrel{\phi}{\rightarrow}\left\{X_{\alpha}\right\}$ be a cofibrant replacement in the projective model category of $I$-shaped diagrams of spectra, so that $\phi$ is a levelwise acyclic fibration. Then each spectrum $\widetilde{X}_{\alpha}$ are (positive) fibrant, and we have

$$
\underset{\alpha}{\operatorname{hocolim}} X_{\alpha}=\underset{\alpha}{\operatorname{colim}} \tilde{X}_{\alpha} \underset{\simeq}{\stackrel{\phi}{\longrightarrow}} \underset{\alpha}{\operatorname{colim}} X_{\alpha} \text {. }
$$


Corollary 5.3.3. (Positive) fibrant discrete G-spectra are (positive) fibrant as nonequivariant spectra.

Proof. Let $X$ be a (positive) fibrant discrete $G$-spectrum. Let $U$ be an open normal subgroup of $G$. By Proposition 3.3.1(2) (and the obvious analog in the positive fibrant case), $X$ is (positive) fibrant as a discrete $U$-spectrum. Therefore, by Lemma 3.1.1 (Corollary 5.2.4), the $U$-fixed points $X^{U}$ are (positive) fibrant as a nonequivariant spectrum. The formula

$$
X=\operatorname{colim}_{U \unlhd_{o} G} X^{U}
$$

shows that $X$ is (positive) fibrant as a nonequivariant spectrum.

Lemma 5.3.4. Filtered colimits in both the category of A-modules and in the category of commutative A-algebras are formed in the category of spectra.

Proof. We treat the case of commutative $A$-algebras; the case of $A$-modules is similar. Let $\left\{E_{\alpha}\right\}$ be a filtered diagram of commutative $A$-algebras. Then the colimit in the category of spectra is easily seen to have the structure of a commutative $A$-algebra with multiplication given by

$$
\begin{aligned}
\left(\operatorname{colim}_{\alpha} E_{\alpha}\right) \wedge_{A}\left(\operatorname{colim}_{\beta} E_{\beta}\right) & \cong \operatorname{colim}_{\alpha} \operatorname{colim}_{\beta} E_{\alpha} \wedge_{A} E_{\beta} \\
& \cong \operatorname{colim}_{\alpha} E_{\alpha} \wedge_{A} E_{\alpha} \\
& \rightarrow \operatorname{colim}_{\alpha} E_{\alpha} .
\end{aligned}
$$

This filtered colimit is easily seen to satisfy the universal property.

We shall henceforth always form filtered colimits of spectra, with the understanding that we implicitly take functorial (positive) fibrant replacements before computing the filtered colimit if the terms in the colimit are not already (positive) fibrant. Therefore, Corollary 5.3.2 implies that our filtered colimits will always have the desired homotopy invariance, so we will never need to take a filtered homotopy colimit of spectra.

If $\left\{X_{\alpha}\right\}$ is a filtered diagram of discrete commutative $G$-A-algebras, then whenever we are taking the filtered colimit, we shall implicitly be taking the filtered colimit $\operatorname{colim}_{\alpha}\left(X_{\alpha}\right)_{f G A-A l g}$ of the functorial fibrant replacements. Note that the underlying spectrum of each $\left(X_{\alpha}\right)_{f G A-A l g}$ is positive fibrant.

Since we often take filtered colimits of commutative $A$-algebras, in the next result (whose proof is similar to that of Corollary 5.3.2), we point out a nice relationship between this colimit and the homotopy colimit in $\mathrm{Alg}_{A}$. This result is also useful in relating the filtered colimits of Section 8 to the homotopy colimits of commutative $S$-algebras that appear in [9, Def. 1.5, Sec. 6].

Lemma 5.3.5. Suppose that $\left\{E_{\alpha}\right\}$ is a filtered diagram of fibrant commutative $A$-algebras. Then there is an equivalence

$$
\underset{\alpha}{\operatorname{colim}} E_{\alpha} \simeq \underset{\alpha}{\operatorname{hocolim}}{ }^{\mathrm{Alg}} E_{\alpha},
$$

where the homotopy colimit hocolim ${ }^{\mathrm{Alg}}$ is taken in the category of commutative A-algebras. 
5.4. Commutative hypercohomology algebras. Let $E$ be a discrete commutative $G$ - $A$-algebra. For any finite set $K$, the mapping spectrum $\operatorname{Map}(K, E)$ is naturally a commutative $A$-algebra, by using the diagonal on $K$. Therefore, by Lemma 5.3.4, the continuous mapping spectrum

$$
\operatorname{Map}^{c}(G, E)=\underset{U \unlhd_{0} G}{\operatorname{colim}} \operatorname{Map}(G / U, E)
$$

is a commutative $A$-algebra.

Since the category of spectra with the positive model structure is Quillen equivalent to the category of spectra with the stable model structure [29, Prop. 14.6], there is an equivalence

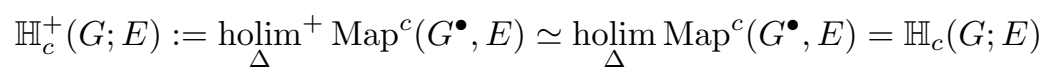

between the homotopy limits computed in the positive and stable model structures. Since the homotopy limit of commutative $A$-algebras is computed in the underlying category of spectra with the positive model structure, we have the following lemma.

Lemma 5.4.1. The hypercohomology spectrum $\mathbb{H}_{c}(G ; E)$ is equivalent to a commutative A-algebra $\mathbb{H}_{c}^{+}(G ; E)$.

When we take hypercohomology spectra of discrete commutative $G$ - $A$-algebras, we shall always implicitly be taking the homotopy limit with respect to the positive model structure.

\section{Profinite Galois extensions}

Although the homotopy limit of $k$-local objects is $k$-local, it is not true in general that $k$-localization commutes with homotopy limits. We begin this section by explaining how, under a certain hypothesis, Assumption 1.0 .3 allows us to commute these two functors. We then explain how a profinite Galois extension of a commutative symmetric ring spectrum $A$ naturally gives rise to a discrete commutative $G$ - $A$ algebra, and we show that our consistency hypothesis allows us to recover the intermediate finite Galois extensions using the homotopy fixed point construction.

6.1. Properties of $k$-localization. Recall that we assume that the $k$-localization functor is given by $\left((-)_{T}\right)_{M}$, where localization with respect to $T$ is smashing and $M$ is a finite spectrum (Assumption 1.0.3). These localizations are the functorial fibrant replacements in appropriately localized model categories. In this subsection we shall establish some lemmas concerning such $k$-localizations.

The following lemma is immediate.

Lemma 6.1.1. If $X$ is a k-local spectrum, then it is a T-local spectrum.

Lemma 6.1.2. Let $\left\{X_{i}\right\}$ be a diagram of spectra. Then there is an equivalence

$$
\left(\operatorname{holim}_{i} X_{i}\right)_{M} \simeq \underset{i}{\operatorname{holim}}\left(X_{i}\right)_{M} .
$$

Proof. The homotopy limit $\operatorname{holim}_{i}\left(X_{i}\right)_{M}$ is $M$-local, so there is a map

$$
f:\left(\operatorname{holim}_{i} X_{i}\right)_{M} \rightarrow \underset{i}{\operatorname{holim}}\left(X_{i}\right)_{M} \text {. }
$$


Smashing with $M$, and using the fact that $M$ is a finite complex, we have the following commutative diagram of equivalences:

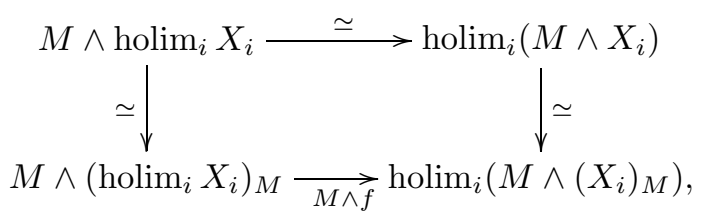

from which we deduce that $f$ is an $M$-local equivalence. Since $f$ is a map between $M$-local spectra, the map $f$ is an equivalence.

Arbitrary localizations do not commute with homotopy limits. Our reason for making Assumption 1.0.3 on $k$-localization is that it allows us to deduce the following corollary.

Corollary 6.1.3. Let $\left\{X_{i}\right\}$ be a diagram of T-local spectra. Then there is an equivalence

$$
\left(\operatorname{holim}_{i} X_{i}\right)_{k} \simeq \underset{i}{\operatorname{holim}}\left(X_{i}\right)_{k} .
$$

Since $T$-localization is smashing, it possesses the following pleasant properties.

\section{Lemma 6.1.4.}

(1) Colimits of T-local spectra are T-local.

(2) If $X$ is a T-local spectrum and $Y$ is any spectrum, then $X \wedge Y$ is T-local.

(3) If $X$ is T-local, then $\operatorname{Map}^{c}(G, X)$ is T-local.

Lemma 6.1.5. Suppose that $f: X \rightarrow Y$ is a $k$-local equivalence of $T$-local discrete $G$-spectra. Then the induced map

$$
f_{*}: \mathbb{H}_{c}(G ; X)_{k} \rightarrow \mathbb{H}_{c}(G ; Y)_{k}
$$

is an equivalence.

Proof. Using Lemma 6.1.4, we see that the hypercohomology functor

$$
\mathbb{H}_{c}(G ;-)=\underset{\Delta}{\operatorname{holim}} \operatorname{Map}^{c}\left(G^{\bullet},-\right)
$$

sends $T$-local spectra to $T$-local spectra. Therefore, we just need to check that the map

$$
f_{*}: \mathbb{H}_{c}(G ; X) \rightarrow \mathbb{H}_{c}(G ; Y)
$$

is an $M$-local equivalence. Since $M$ is finite and $f$ is an $M$-local equivalence, we have

$$
\begin{array}{ccc}
M \wedge \mathbb{H}_{c}(G ; X) & \simeq & \mathbb{H}_{c}(G ; M \wedge X) \\
\stackrel{(M \wedge f)_{*}}{\longrightarrow} & \mathbb{H}_{c}(G ; M \wedge Y) \\
\simeq & & M \wedge \mathbb{H}_{c}(G ; Y),
\end{array}
$$

which implies that $M \wedge f_{*}$ is an equivalence.

Theorem 3.2.1 implies the following corollary.

Corollary 6.1.6. Suppose that $G$ has finite vcd and that $f: X \rightarrow Y$ is a $k$-local equivalence of T-local discrete $G$-spectra. Then the induced map

$$
f_{*}: X^{h G} \rightarrow Y^{h G}
$$

is a k-local equivalence. 
We end this section by briefly explaining the relationship between localized fixed points, and the right derived functor of fixed points taken with respect to a localized model structure on discrete $G$-spectra.

Let $\mathcal{C}_{k}$ be the class of morphisms in $\Sigma \mathrm{Sp}_{G}$ which are $k$-local equivalences on the underlying symmetric spectra. Let $\left(\Sigma \mathrm{Sp}_{G}\right)_{k}$ be the model category obtained by localizing $\Sigma \mathrm{Sp}_{G}$ at $\mathcal{C}_{k}$. Such a localized model category exists: the arguments of Bousfield [4] carry over in our setting to show that the $k$-local equivalences in $\Sigma \operatorname{Sp}_{G}$ are the class of $f$-local equivalences for some map $f$, and the localized model structure exists since the model structure on $\Sigma \mathrm{Sp}_{G}$ is left proper and cellular (see, for instance, [17, Thm. 4.1.1]). The $k$-local model structure $\left(\Sigma \mathrm{Sp}_{G}\right)_{k}$ is characterized by:

- the cofibrations are the underlying cofibrations of symmetric spectra,

- the weak equivalences are the underlying $k$-local equivalences of symmetric spectra,

- the fibrations are determined.

We shall let

$$
\alpha_{G, k, X}: X \rightarrow X_{f_{k} G}
$$

denote a fibrant replacement functor in $\left(\Sigma \mathrm{Sp}_{G}\right)_{k}$.

The pair of functors $\left(\operatorname{triv},(-)^{G}\right)$ forms a Quillen pair on the localized model categories

$$
\text { triv }: \Sigma \mathrm{Sp}_{k} \rightleftarrows\left(\Sigma \mathrm{Sp}_{G}\right)_{k}:(-)^{G}
$$

since triv preserves cofibrations and $k$-local equivalences. We shall define $(-)^{h_{k} G}$ to be the Quillen right derived functor of $(-)^{G}$ with respect to the $k$-local model structure:

$$
X^{h_{k} G}=\left(X_{f_{k} G}\right)^{G}
$$

Proposition 6.1.7. Let $X$ be a discrete $G$-spectrum.

(1) For each open subgroup $U$ contained in $G$, the spectrum $X^{h_{k} U}$ is $k$-local.

(2) The spectrum $X_{f_{k} G}$ is T-local.

(3) Suppose $G$ has finite vcd and $X$ is T-local. Then the map

$$
\left(X^{h G}\right)_{k} \rightarrow X^{h_{k} G}
$$

is an equivalence.

Proof. Observe that the same arguments used in the proof of Proposition 3.3.1 apply to show that the adjunction

$$
\operatorname{Ind}_{U}^{G}:\left(\Sigma \mathrm{Sp}_{U}\right)_{k} \rightleftarrows\left(\Sigma \mathrm{Sp}_{G}\right)_{k}: \operatorname{Res}_{G}^{U}
$$

is a Quillen pair with respect to the $k$-local model structures. In particular, $\operatorname{Res}_{G}^{U}$ preserves $k$-locally fibrant objects, so $X_{f_{k} G}$ is $k$-locally fibrant as a discrete $U$ spectrum. Since the fixed point functor

$$
(-)^{U}:\left(\Sigma \mathrm{Sp}_{U}\right)_{k} \rightarrow \Sigma \mathrm{Sp}_{k}
$$

is a right Quillen functor, it follows that

$$
\left(X_{f_{k} G}\right)^{U}=X^{h_{k} U}
$$

is $k$-local. This establishes (1). (2) then follows immediately from Lemma 6.1.4 since there is an isomorphism

$$
X_{f_{k} G} \cong \operatorname{colim}_{U \leq o}\left(X_{f_{k} G}\right)^{U}
$$


The map in (3) arises from the fact that $X_{f_{k} G}$ is fibrant as a discrete $G$-spectrum; hence there is a $k$-local equivalence

$$
X_{f G} \rightarrow X_{f_{k} G} .
$$

Note that if $X$ is assumed to be $T$-local, then $X_{f G}$ must also be $T$-local, since it is equivalent to $X$. Consider the following diagram:

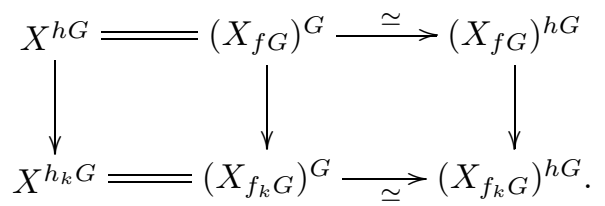

The rightmost vertical arrow is a $k$-local equivalence by Corollary 6.1.6. Therefore the leftmost vertical arrow is a $k$-local equivalence, and the induced morphism

$$
\left(X^{h G}\right)_{k} \rightarrow X^{h_{k} G}
$$

is an equivalence.

Remark 6.1.8. Although the underlying nonequivariant spectrum of $X_{f_{k} G}$ is $T$ local, it is not, in general, $k$-local. As pointed out in the proof of Proposition 6.1.7. the spectrum $X_{f_{k} G}$ is a filtered colimit of $k$-local spectra.

We record the following proposition, whose proof is identical to the proof of Proposition 3.3.1.

Proposition 6.1.9. Let $X$ be a discrete $G$-spectrum, and suppose that $U$ is an open subgroup of $G$.

(1) If $U$ is normal in $G$, the $U$-fixed point spectrum $\left(X_{f_{k} G}\right)^{U}$ is $k$-locally fibrant as a discrete $G / U$-spectrum.

(2) The $k$-locally fibrant discrete $G$-spectrum $X_{f_{k} G}$ is $k$-locally fibrant as a discrete $U$-spectrum.

(3) If $U$ is normal in $G$, the homotopy fixed point spectrum $X^{h_{k} U}$ is a $G / U$ spectrum.

(4) If $U$ is normal in $G$, there is an equivalence $X^{h_{k} G} \simeq\left(X^{h_{k} U}\right)^{h_{k} G / U}$.

6.2. Profinite Galois extensions as discrete $G$-spectra. We first give the definition of a profinite Galois extension, which is a slight modification of the notion of a pro-G-Galois extension, due to John Rognes (see [34, Section 8.1]). Let $A$ be a $k$-local cofibrant commutative symmetric ring spectrum, let $E$ be a commutative $A$-algebra, and let $G$ be a profinite group.

Definition 6.2.1 (Profinite Galois extension). The spectrum $E$ is a (profaithful) $k$-local $G$-Galois extension of $A$ if

(1) there is a directed system of (faithful) finite $k$-local $G / U_{\alpha}$-Galois extensions $E_{\alpha}$ of $A$, for $\left\{U_{\alpha}\right\}$ a cofinal system of open normal subgroups of $G$;

(2) all of the maps $E_{\alpha} \rightarrow E_{\beta}$ are $G$-equivariant and are cofibrations of underlying commutative $A$-algebras;

(3) for $\alpha \leq \beta$, letting $K_{\alpha, \beta}$ denote the quotient $U_{\alpha} / U_{\beta}$, the natural maps $E_{\alpha} \rightarrow E_{\beta}^{h K_{\alpha, \beta}}$ are equivalences;

(4) the spectrum $E$ is the filtered colimit $\operatorname{colim}_{\alpha} E_{\alpha}$. 
Remark 6.2.2. The spectra $E_{\alpha}$ are $k$-local, but the spectrum $E$ need not be $k$-local. However, Assumption 1.0 .3 does imply that $E$ is $T$-local.

Proposition 6.2.3. The spectrum $E$ in Definition 6.2.1 is a discrete commutative $G$-A-algebra.

Proof. Clearly, $E_{\alpha}$ is a discrete commutative $G$ - $A$-algebra. Discrete commutative $G$ - $A$-algebras are closed under filtered colimits taken in the category of commutative $A$-algebras.

Proposition 6.2.4 (Rognes [34, Sec. 8.1]). If $E$ is a k-local G-Galois extension of $A$, then there are natural equivalences:

$$
\begin{gathered}
\left(E \wedge_{A} E\right)_{k} \stackrel{\simeq}{\rightarrow}\left(\operatorname{Map}^{c}(G, E)\right)_{k}, \\
(E[[G]])_{k} \simeq F_{A}\left(E_{k}, E_{k}\right) .
\end{gathered}
$$

6.3. The consistent hypothesis. In this subsection, we assume that $E$ is a $k$ local profinite $G$-Galois extension of $A$. We recall from the Introduction some terminology:

- $E$ is consistent over $A$ if the map $A \rightarrow A_{k, E}^{\wedge}$ is an equivalence, and

- $E$ has finite vcd if the profinite group $G$ has finite virtual cohomological dimension.

Proposition 6.3.1. Let $E$ be a $k$-local profinite $G$-Galois extension of $A$ of finite $v c d$. Then there is a natural equivalence

$$
A_{k, E}^{\wedge} \simeq\left(E^{h G}\right)_{k}
$$

between the $k$-local Amitsur derived completion and the $k$-localization of the homotopy fixed point spectrum.

Proof. By iterating Proposition 6.2.4, the natural map

$$
(\underbrace{E \wedge_{A} E \wedge_{A} \cdots \wedge_{A} E}_{n+1})_{k} \rightarrow\left(\operatorname{Map}^{c}\left(G^{n}, E\right)\right)_{k}
$$

is an equivalence. Totalizing the associated cosimplicial spectra and using Corollary 6.1 .3 and Theorem 3.2.1, we have:

$$
\begin{aligned}
A_{k, E}^{\wedge} & =\operatorname{holim}_{\Delta}\left(E^{\wedge{ }_{A} \bullet+1}\right)_{k} \\
& \simeq \operatorname{holim}_{\Delta}\left(\operatorname{Map}^{c}\left(G^{\bullet}, E\right)\right)_{k} \\
& \simeq\left(\operatorname{holim}_{\Delta} \operatorname{Map}^{c}\left(G^{\bullet}, E\right)\right)_{k} \\
& \simeq\left(E^{h G}\right)_{k} .
\end{aligned}
$$

Corollary 6.3.2. Let $E$ be a $k$-local profinite $G$-Galois extension of $A$ of finite vcd. Then the extension is consistent if and only if the A-algebra unit map

$$
A \rightarrow\left(E^{h G}\right)_{k}
$$

is an equivalence. 
We shall say that a $k$-local $A$-module $X$ is $k$-locally dualizable if the map

$$
\left(D_{A}(X) \wedge_{A} X\right)_{k} \rightarrow F_{A}(X, X)
$$

is an equivalence. Here, $D_{A}(-)=F_{A}(-, A)$ is the Spanier-Whitehead dual in the category of $A$-modules. The following standard properties of $k$-local dualizability are contained in [34, Lem. 3.3.2(a),(b)].

\section{Lemma 6.3.3.}

(1) For $k$-local $A$-modules $X, Y$, and $Z$, the natural map

$$
\left(F_{A}(X, Y) \wedge_{A} Z\right)_{k} \rightarrow F_{A}\left(X,\left(Y \wedge_{A} Z\right)_{k}\right)
$$

is an equivalence if either $X$ or $Z$ is $k$-locally dualizable.

(2) If the $k$-local $A$-module $X$ is $k$-locally dualizable, then $D_{A}(X)$ is also $k$ locally dualizable, and the natural map

$$
X \rightarrow D_{A}\left(D_{A}(X)\right)
$$

is an equivalence.

We note the following useful consequence of $k$-local dualizability which makes use of Assumption 1.0 .3

Lemma 6.3.4. Suppose that $X$ is a k-local A-module which is $k$-locally dualizable, and that $\left\{Y_{i}\right\}$ is a diagram of T-local A-modules. Then the natural map

$$
\left(X \wedge_{A} \operatorname{holim}_{i} Y_{i}\right)_{k} \rightarrow\left(\operatorname{holim}_{i} X \wedge_{A} Y_{i}\right)_{k}
$$

is an equivalence.

Proof. The result follows from the following chain of equivalences:

$$
\begin{aligned}
\left(X \wedge_{A} \operatorname{holim}_{i} Y_{i}\right)_{k} & \simeq\left(D_{A}\left(D_{A}(X)\right) \wedge_{A} \underset{i}{\operatorname{holim}} Y_{i}\right)_{k} \\
& \simeq\left(D_{A}\left(D_{A}(X)\right) \wedge_{A}\left(\operatorname{holim}_{i} Y_{i}\right)_{k}\right)_{k} \\
& \simeq F_{A}\left(D_{A}(X),\left(\underset{i}{\left.\left.\operatorname{holim} Y_{i}\right)_{k}\right)}\right.\right. \\
& \simeq F_{A}\left(D_{A}(X), \operatorname{holim}_{i}\left(Y_{i}\right)_{k}\right) \\
& \simeq \operatorname{holim}_{i} F_{A}\left(D_{A}(X),\left(Y_{i}\right)_{k}\right) \\
& \simeq \operatorname{holim}_{i}\left(D_{A}\left(D_{A}(X)\right) \wedge_{A}\left(Y_{i}\right)_{k}\right)_{k} \\
& \simeq \operatorname{holim}_{i}\left(D_{A}\left(D_{A}(X)\right) \wedge_{A} Y_{i}\right)_{k} \\
& \simeq\left(\operatorname{holim}_{i} X \wedge_{A} Y_{i}\right)_{k},
\end{aligned}
$$

where the fourth and last equivalences follow from Corollary 6.1.3.

We shall repeatedly use the following dualizability result [34, Props. 6.2.1, 6.4.7].

Proposition 6.3.5. If $E$ is a finite $k$-local Galois extension of $A$ (not required to be faithful), then $E$ is a k-locally dualizable A-module. Also, there is a natural discriminant map (in the stable homotopy category)

$$
E \rightarrow D_{A}(E)
$$

which is an equivalence. 
Given a $k$-local profinite $G$-Galois extension $E=\operatorname{colim}_{\alpha} E_{\alpha}$ of $A$, each of the spectra $E_{\alpha}$ carries a $G$-action, where the subgroup $U_{\alpha}$ acts trivially on $E_{\alpha}$. Since the maps

$$
E_{\alpha} \rightarrow E_{\beta}
$$

are $G$-equivariant, each of the maps

$$
E_{\alpha} \rightarrow \operatorname{colim}_{\beta} E_{\beta}=E
$$

is $G$-equivariant. Since the subgroup $U_{\alpha}$ acts trivially on $E_{\alpha}$, we get an induced $G / U_{\alpha}$-equivariant map

$$
E_{\alpha} \rightarrow E^{U_{\alpha}} \rightarrow\left(E_{f G A-\mathrm{Alg}}\right)^{U_{\alpha}} \simeq E^{h U_{\alpha}},
$$

where the last equivalence follows from Proposition 3.3 .1 .

Being consistent implies the following consistency result.

Lemma 6.3.6. Suppose that $E=\operatorname{colim}_{\alpha} E_{\alpha}$ is a consistent $k$-local profinite $G$ Galois extension of finite $v c d$. Then for each $\alpha$, the natural $G / U_{\alpha}$-equivariant map

$$
E_{\alpha} \rightarrow\left(\left(E_{f G A-\mathrm{Alg}}\right)^{U_{\alpha}}\right)_{k} \simeq\left(E^{h U_{\alpha}}\right)_{k}
$$

is an equivalence.

Proof. Since $E_{\alpha}$ is a $k$-local $G / U_{\alpha}$-Galois extension of $A$, we have a chain of equivalences:

$$
\begin{aligned}
\left(E_{\alpha} \wedge_{A} E\right)_{k} & \simeq\left(E_{\alpha} \wedge_{A} \operatorname{colim}_{\beta \geq \alpha} E_{\beta}\right)_{k} \\
& \simeq\left(\operatorname{colim}_{\beta \geq \alpha}\left(\left(E_{\alpha} \wedge_{A} E_{\alpha}\right) \wedge_{E_{\alpha}} E_{\beta}\right)\right)_{k} \\
& \simeq\left(\operatorname{colim}_{\beta \geq \alpha}\left(\operatorname{Map}\left(G / U_{\alpha}, E_{\alpha}\right) \wedge_{E_{\alpha}} E_{\beta}\right)\right)_{k} \\
& \simeq\left(\operatorname{colim}_{\beta \geq \alpha} \operatorname{Map}\left(G / U_{\alpha}, E_{\beta}\right)\right)_{k} \\
& \simeq\left(\operatorname{Map}\left(G / U_{\alpha}, E\right)\right)_{k},
\end{aligned}
$$

where the $G$-action on the factor $E$ in $\left(E_{\alpha} \wedge_{A} E\right)_{k}$ corresponds to the conjugation action on $\left(\operatorname{Map}\left(G / U_{\alpha}, E\right)\right)_{k}$. By Corollary 6.3.2, the natural map

$$
A \rightarrow\left(E^{h G}\right)_{k}
$$

is an equivalence. Smashing (6.1) over $A$ with $E_{\alpha}$, using Theorem 3.2.1, employing the fact that $E_{\alpha}$ is a $k$-locally dualizable $A$-module (Proposition 6.3.5] and Lemma 6.3.4), and applying Corollary 6.1.3 and Shapiro's Lemma (3.4.2), we have 
the following equivalences:

$$
\begin{aligned}
& E_{\alpha} \simeq\left(E_{\alpha} \wedge_{A} A\right)_{k} \\
& \simeq\left(E_{\alpha} \wedge_{A} E^{h G}\right)_{k} \\
& \simeq\left(E_{\alpha} \wedge_{A} \operatorname{holim}_{\Delta} \operatorname{Map}^{c}\left(G^{\bullet}, E\right)\right)_{k} \\
& \simeq\left(\operatorname{holim}_{\Delta} \operatorname{Map}^{c}\left(G^{\bullet}, E_{\alpha} \wedge_{A} E\right)\right)_{k} \\
& \simeq \underset{\Delta}{\operatorname{holim}} \operatorname{Map}^{c}\left(G^{\bullet}, E_{\alpha} \wedge_{A} E\right)_{k} \\
& \simeq \operatorname{holim}_{\triangle} \operatorname{Map}^{c}\left(G^{\bullet}, \operatorname{Map}\left(G / U_{\alpha}, E\right)\right)_{k} \\
& \simeq\left(\underset{\Delta o l i m}{\operatorname{Map}^{c}}\left(G^{\bullet}, \operatorname{Map}\left(G / U_{\alpha}, E\right)\right)\right)_{k} \\
& \simeq\left(\operatorname{Map}\left(G / U_{\alpha}, E\right)^{h G}\right)_{k} \\
& \simeq\left(E^{h U_{\alpha}}\right)_{k} \text {. }
\end{aligned}
$$

Adding the profaithful hypothesis allows us to expand the particular system $\left\{U_{\alpha}\right\}$ of open normal subgroups of $G$ to the collection of all open normal subgroups of $G$.

Proposition 6.3.7. Let $E$ be a consistent profaithful k-local profinite $G$-Galois extension of $A$ of finite $v c d$.

(1) For each open normal subgroup $U$ of $G,\left(E^{h U}\right)_{k}$ is a faithful $k$-local $G / U$ Galois extension of $A$.

(2) If $U \leq V$ are a pair of open subgroups of $G$ with $U$ normal in $V$, then $\left(E^{h U}\right)_{k}$ is a faithful $k$-local $V / U$-Galois extension of $\left(E^{h V}\right)_{k}$.

Proof. For both parts, we repeatedly use the fundamental theorem of Galois theory [34, Thm. 1.2].

(1) Choose $\alpha$ so that $U_{\alpha}$ is contained in $U$. Then by Lemma 6.3.6, there is an equivalence $E_{\alpha} \simeq\left(E^{h U_{\alpha}}\right)_{k}$, so $\left(E^{h U_{\alpha}}\right)_{k}$ is a faithful $k$-local $G / U_{\alpha}$-Galois extension of $A$. Proposition 3.3 .1 implies that there are equivalences

$$
\left(E^{h U}\right)_{k} \simeq\left(\left(E^{h U_{\alpha}}\right)^{h U / U_{\alpha}}\right)_{k} \simeq\left(\left(E^{h U_{\alpha}}\right)_{k}\right)^{h U / U_{\alpha}} .
$$

Therefore, the fundamental theorem of Galois theory implies that $\left(E^{h U}\right)_{k}$ is a faithful $k$-local $G / U$-Galois extension of $A$.

(2) Let $N$ be an open normal subgroup of $G$ contained in $U$. By (1), we know that $\left(E^{h N}\right)_{k}$ is a faithful $k$-local $G / N$-Galois extension of $A$. By Proposition 3.3.1. we have

$$
\left(E^{h V}\right)_{k} \simeq\left(\left(E^{h N}\right)^{h V / N}\right)_{k} \simeq\left(\left(E^{h N}\right)_{k}\right)^{h V / N} .
$$

Thus, Galois theory implies that $\left(E^{h N}\right)_{k}$ is a faithful $k$-local $V / N$-Galois extension of $\left(E^{h V}\right)_{k}$. As before, we have

$$
\left(E^{h U}\right)_{k} \simeq\left(\left(E^{h N}\right)_{k}\right)^{h U / N} .
$$

Since $U / N$ is normal in $V / N$, with quotient $V / U$, Galois theory implies that $\left(E^{h U}\right)_{k}$ is a faithful $k$-local $V / U$-Galois extension of $\left(E^{h V}\right)_{k}$. 


\section{Closed homotopy fixed points of profinite Galois extensions}

Let $E$ be a consistent profaithful $k$-local profinite $G$-Galois extension of $A$ of finite vcd. We begin by showing that under these hypotheses, the $H$-homotopy fixed points functor is well-behaved when $H$ is any closed subgroup of $G$. We then prove the forward part of the profinite Galois correspondence, and we compute the homotopy type of the function spectrum between arbitrary $k$-local closed homotopy fixed point spectra of $E$.

7.1. Iterated Galois homotopy fixed points. In this subsection we will extend the results of Section 3.5 to all closed subgroups $H$ of $G$. Let $j: H \hookrightarrow G$ be the inclusion of the closed subgroup $H$. Also, recall that, by hypothesis, $G$ has finite vcd. Then we prove the following theorem and derive consequences from it.

Theorem 7.1.1. The natural map

$$
\left(\operatorname{colim}_{H \leq U \leq o} E^{h U}\right)_{k} \rightarrow\left(E^{h H}\right)_{k}
$$

is an equivalence.

In order to prove Theorem 7.1.1, we need to introduce a spectrum $E^{\prime}$ which is equivalent to $E$, but which has better point-set level properties. Observe that by Proposition 6.3.7. the collection of homotopy fixed point spectra $\left\{\left(E^{h U}\right)_{k}\right\}_{U \unlhd_{o} G}$ gives rise to a $k$-local profinite $G$-Galois extension

$$
E^{\prime}=\operatorname{colim}_{U \unlhd_{o} G}\left(E^{h U}\right)_{k}
$$

of $A$.

Strictly speaking, given an open subgroup $V$ of $G$, the spectrum $E$ is not an $\left(E^{h V}\right)_{k}$-algebra. Since the spectrum $\left(E^{h U}\right)_{k}$ is an $\left(E^{h V}\right)_{k}$-algebra for every open normal subgroup $U$ of $G$ contained in $V$, the spectrum $E^{\prime}$ is an $\left(E^{h V}\right)_{k}$-algebra. Furthermore, by Lemma 6.3.6. the map

$$
E=\operatorname{colim}_{\alpha} E_{\alpha} \rightarrow \underset{\alpha}{\operatorname{colim}}\left(E^{h U_{\alpha}}\right)_{k} \cong E^{\prime}
$$

is an equivalence of discrete commutative $G$ - $A$-algebras.

We shall need the following fundamental lemma.

Lemma 7.1.2. Let $V$ be an open normal subgroup of $G$. Then the natural map

$$
\left(\left(E^{h V}\right)_{k} \wedge_{\left(E^{h H V}\right)_{k}}\left(E^{\prime}\right)^{h H}\right)_{k} \rightarrow\left(\left(E^{\prime}\right)^{h(H \cap V)}\right)_{k},
$$

induced from the commutative diagram

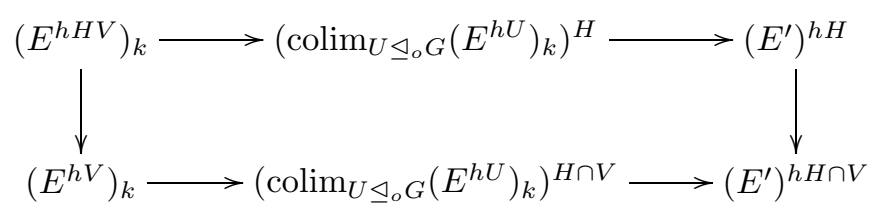

of commutative symmetric ring spectra, is an equivalence.

Proof. The lemma will be proven by showing that there exists a zig-zag of $k$-local equivalences between

$$
\left(E^{h V}\right)_{k} \wedge\left(E^{h H V}\right)_{k}\left(E^{\prime}\right)^{h H} \quad \text { and } \quad\left(E^{\prime}\right)^{h H \cap V},
$$


which are maps both of commutative $\left(E^{h V}\right)_{k}$-algebras and commutative $\left(E^{\prime}\right)^{h H_{-}}$ algebras. Let $Q$ be the finite group $H V / V \cong H /(H \cap V)$. Note that, by Proposition 6.3.7, the map

$$
\left(E^{h H V}\right)_{k} \rightarrow\left(E^{h V}\right)_{k}
$$

is a $k$-local $Q$-Galois extension.

Observe that there is a zig-zag of maps:

$$
\begin{aligned}
\left(E^{h V}\right)_{k} \wedge_{\left(E^{h H V}\right)_{k}}\left(E^{\prime}\right)^{h H} & =\left(E^{h V}\right)_{k} \wedge_{\left(E^{h H V}\right)_{k}}\left(E_{f H}^{\prime}\right)^{H} \\
& \stackrel{w_{1}}{\longrightarrow}\left(\left(E^{h V}\right)_{k} \wedge_{\left(E^{h H V}\right)_{k}} E_{f H}^{\prime}\right)^{H} \\
& \stackrel{w_{2}}{\longrightarrow}\left(\left(E^{h V}\right)_{k} \wedge_{\left(E^{h H V}\right)_{k}} E_{f H}^{\prime}\right)^{h H} \\
& \simeq \\
\simeq & \left(\left(E^{h V}\right)_{k} \wedge_{\left(E^{h H V}\right)_{k}} E^{\prime}\right)^{h H} .
\end{aligned}
$$

The map $w_{2}$ above is the inclusion of fixed points into homotopy fixed points. Each of the maps above is a map of commutative $\left(E^{h V}\right)_{k}$-algebras and commutative $\left(E^{\prime}\right)^{h H}$-algebras. Furthermore, the composite $w=w_{2} \circ w_{1}$ is a $k$-local equivalence, since we have a commutative diagram (in the $k$-local stable homotopy category)

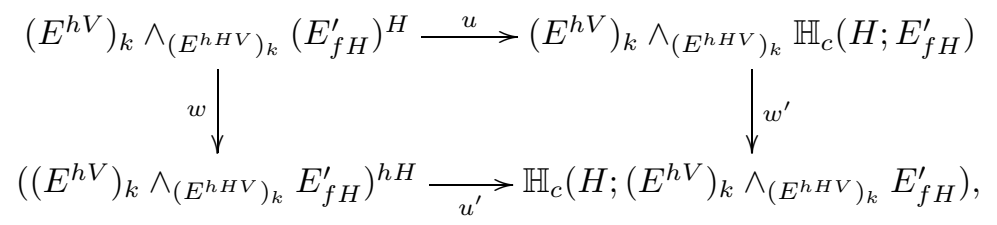

where the maps $u$ and $u^{\prime}$ are equivalences by Theorem 3.2.1 and the map $w^{\prime}$ is seen to be a $k$-local equivalence by using the fact that $\left(E^{h V}\right)_{k}$ is a $k$-locally dualizable $\left(E^{h H V}\right)_{k}$-module (Proposition 6.3.5 and Lemma 6.3.4).

The composite

$$
\begin{aligned}
\left(E^{h V}\right)_{k} \wedge_{\left(E^{h H V}\right)_{k}} E^{\prime} & \cong\left(\left(E^{h V}\right)_{k} \wedge_{\left(E^{h H V}\right)_{k}}\left(E^{h V}\right)_{k}\right) \wedge_{\left(E^{h V}\right)_{k}} E^{\prime} \\
& \rightarrow \operatorname{Map}\left(Q,\left(E^{h V}\right)_{k}\right) \wedge_{\left(E^{h V}\right)_{k}} E^{\prime} \\
& \simeq \operatorname{Map}\left(Q, E^{\prime}\right)
\end{aligned}
$$

is a $k$-local equivalence. Here, the smash product $\wedge_{\left(E^{h V}\right)_{k}}$ on the right-hand side of the first line of (7.1) uses the left $\left(E^{h V}\right)_{k}$-module structure on $\left(E^{h V}\right)_{k} \wedge\left(E^{h H V}\right)_{k}$ $\left(E^{h V}\right)_{k}$. Under the isomorphism

$$
E^{\prime} \cong\left(E^{h V}\right)_{k} \wedge\left(E^{h V}\right)_{k} E^{\prime}
$$

the $G$-action on $E^{\prime}$ is transformed to the diagonal action on $\left(E^{h V}\right)_{k} \wedge\left(E^{h V}\right)_{k} E^{\prime}$. Therefore, under (7.1), the $H$-action on $E^{\prime}$ is transformed to the conjugation action on $\operatorname{Map}\left(Q, E^{\prime}\right)$. Furthermore, under (7.1):

(1) the $E^{\prime}$-algebra structure on $\left(E^{h V}\right)_{k} \wedge_{\left(E^{h H V}\right)_{k}} E^{\prime}$ is sent to that given by the inclusion

$$
E^{\prime} \rightarrow \operatorname{Map}\left(Q, E^{\prime}\right)
$$

of the constant maps, and

(2) the $\left(E^{h V}\right)_{k}$-module structure on $\left(E^{h V}\right)_{k} \wedge_{\left(E^{h H V}\right)_{k}} E^{\prime}$ is sent to that given by the composite

$$
\left(E^{h V}\right)_{k} \stackrel{\xi}{\rightarrow} \operatorname{Map}\left(Q,\left(E^{h V}\right)_{k}\right) \rightarrow \operatorname{Map}\left(Q, E^{\prime}\right),
$$

where $\xi$ is the adjoint of the $Q$-action map. 
Taking $H$-homotopy fixed points of (7.1) gives, by Corollary 6.1.6, a $k$-local equivalence

$$
\left(\left(E^{h V}\right)_{k} \wedge\left(E^{h H V}\right)_{k} E^{\prime}\right)^{h H} \rightarrow \operatorname{Map}\left(Q, E^{\prime}\right)^{h H},
$$

which is a map of commutative $\left(E^{h V}\right)_{k}$-algebras and of commutative $\left(E^{\prime}\right)^{h H_{-}}$ algebras. The proof of the lemma is completed by observing that the equivalence given by Shapiro's Lemma (Lemma 3.4.2)

$$
\left(E^{\prime}\right)^{h H \cap V} \stackrel{\simeq}{\rightarrow} \operatorname{Map}\left(H /(H \cap V), E^{\prime}\right)^{h H} \cong \operatorname{Map}\left(Q, E^{\prime}\right)^{h H}
$$

is a map of commutative $\left(E^{h V}\right)_{k}$-algebras and of commutative $\left(E^{\prime}\right)^{h H}$-algebras.

Proof of Theorem 7.1.1. Choose an open normal subgroup $V$ of $G$ of finite cohomological dimension. By Proposition 3.5.3, we see that the map

$$
\operatorname{colim}_{H \leq U \leq o H V} E^{h(U \cap V)} \rightarrow E^{h(H \cap V)}
$$

is an equivalence. Let $Q=H V / V \cong H /(H \cap V)$ be the finite quotient group. For each open subgroup $U$ of $H V$ containing $H$, we have $U V=H V$. Therefore, there is an isomorphism $Q=U V / V \cong U /(U \cap V)$. By Proposition 6.3.7, the extensions

$$
\begin{gathered}
\left(E^{h U}\right)_{k} \rightarrow\left(E^{h(U \cap V)}\right)_{k}, \\
\left(E^{h U V}\right)_{k} \rightarrow\left(E^{h V}\right)_{k}
\end{gathered}
$$

are faithful $k$-local $Q$-Galois extensions. Therefore, by Remark 1.0 .2 , the norm maps give the following equivalences:

$$
\begin{aligned}
\left(\left(E^{h(U \cap V)}\right)_{h Q}\right)_{k} & \simeq\left(\left(E^{h(U \cap V)}\right)^{h Q}\right)_{k}, \\
\left(\left(E^{h V}\right)_{h Q}\right)_{k} & \simeq\left(\left(E^{h V}\right)^{h Q}\right)_{k} .
\end{aligned}
$$

We may now establish that the natural map

$$
\left(\operatorname{colim}_{H \leq U \leq o} E^{h U}\right)_{k} \rightarrow\left(E^{h H}\right)_{k}
$$

is an equivalence by establishing a chain of intermediate equivalences. Using Proposition 3.3.1, we have

$$
\begin{aligned}
\left(\operatorname{colim}_{H \leq U \leq o} E^{h U}\right)_{k} & \simeq\left(\operatorname{colim}_{H \leq U \leq_{o} H V} E^{h U}\right)_{k} \\
& \simeq\left(\operatorname{colim}_{H \leq U \leq_{o} H V}\left(E^{h(U \cap V)}\right)^{h U /(U \cap V)}\right)_{k} \\
& \cong\left(\operatorname{colim}_{H \leq U \leq_{o} H V}\left(E^{h(U \cap V)}\right)^{h Q}\right)_{k} .
\end{aligned}
$$

Using equivalence (7.3), we have

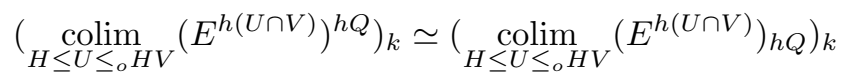

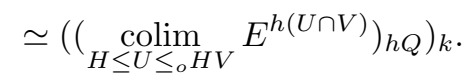

Since $H \cap V$ is a subgroup of $V$, and $V$ has finite cohomological dimension, $H \cap V$ has finite cohomological dimension. Therefore, we may apply Proposition 3.5 .3 to deduce that there is an equivalence

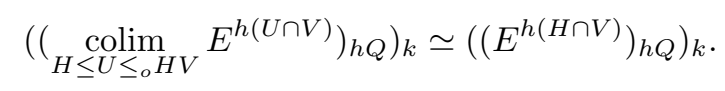


We may now use Lemma 7.1.2 to deduce that there are equivalences

$$
\begin{aligned}
\left(\left(E^{h(H \cap V)}\right)_{h Q}\right)_{k} & \simeq\left(\left(\left(E^{\prime}\right)^{h(H \cap V)}\right)_{h Q}\right)_{k} \\
& \simeq\left(\left(\left(E^{h V}\right)_{k} \wedge\left(E^{h H V}\right)_{k}\left(E^{\prime}\right)^{h H}\right)_{h Q}\right)_{k} \\
& \simeq\left(\left(\left(\left(E^{h V}\right)_{k}\right)_{h Q}\right)_{k} \wedge_{\left(E^{h H V}\right)_{k}}\left(E^{\prime}\right)^{h H}\right)_{k} .
\end{aligned}
$$

Applying equivalence (17.4), and applying Proposition 3.3.1, we have

$$
\begin{aligned}
\left(\left(\left(\left(E^{h V}\right)_{k}\right)_{h Q}\right)_{k} \wedge\left(E^{h H V}\right)_{k}\left(E^{\prime}\right)^{h H}\right)_{k} & \simeq\left(\left(\left(E^{h V}\right)^{h Q}\right)_{k} \wedge\left(E^{h H V}\right)_{k}\left(E^{\prime}\right)^{h H}\right)_{k} \\
& \simeq\left(\left(\left(E^{h V}\right)^{h(H V / V)}\right)_{k} \wedge\left(E^{h H V}\right)_{k}\left(E^{\prime}\right)^{h H}\right)_{k} \\
& \simeq\left(\left(E^{h H V}\right)_{k} \wedge\left(E^{h H V}\right)_{k}\left(E^{\prime}\right)^{h H}\right)_{k} \\
& \simeq\left(\left(E^{\prime}\right)^{h H}\right)_{k} \\
& \simeq\left(E^{h H}\right)_{k},
\end{aligned}
$$

as desired.

Using Corollary 3.5.2, Theorem 7.1.1 has the following corollary.

Corollary 7.1.3. There is an equivalence $\left(\left(E_{f G}\right)^{H}\right)_{k} \simeq\left(E^{h H}\right)_{k}$.

We wish to derive from Corollary 7.1.3 an "iterated homotopy fixed point theorem" analogous to Corollary 3.5.6. The presence of the $k$-localizations in the statement of Corollary 7.1.3 presents some difficulties. One might hope that these could be overcome by using the $k$-local model structures and ask if $X_{f_{k} G}$ is $k$-locally fibrant as a discrete $H$-spectrum. This is typically untrue, however. For instance, if $H=e$, this would imply that the underlying spectrum of $X_{f_{k} G}$ is $k$-local, which is typically false (see Remark 6.1.8).

However, we can still formulate an iterated homotopy fixed point theorem on the level of homotopy categories. We first observe that we have the following $k$-local analog of Proposition 3.5.1.

Proposition 7.1.4. Suppose that $N$ is a closed normal subgroup of $G$. Then the functor

$$
(-)^{N}:\left(\Sigma \mathrm{Sp}_{G}\right)_{k} \rightarrow\left(\Sigma \mathrm{Sp}_{G / N}\right)_{k}
$$

is a right Quillen functor. Let $R(-)^{N}$ denote its right derived functor. Then the following diagram commutes up to natural isomorphism:

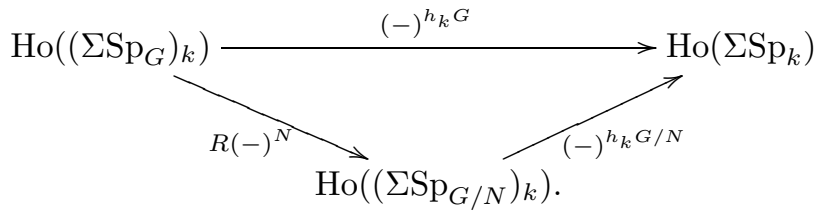

Proof. The adjoint pair $\left(\operatorname{Res}_{G / N}^{G},(-)^{N}\right)$ forms a Quillen pair

$$
\operatorname{Res}_{G / N}^{G}:\left(\Sigma \operatorname{Sp}_{G / N}\right)_{k} \rightleftarrows\left(\Sigma \operatorname{Sp}_{G}\right)_{k}:(-)^{N}
$$

since $\operatorname{Res}_{G / N}^{G}$ is easily seen to preserve cofibrations and $k$-local equivalences. The functor $(-)^{N}$ has a Quillen right derived functor $R(-)^{N}$. For any discrete $G$ spectrum $X$, the functor $R(-)^{N}$ is computed to be

$$
R(X)^{N}=\left(X_{f_{k} G}\right)^{N} .
$$


Since $(-)^{N}$ is a right Quillen functor, $\left(X_{f_{k} G}\right)^{N}$ is $k$-locally fibrant as a discrete $G / N$-spectrum. We therefore have

$$
X^{h_{k} G}=\left(X_{f_{k} G}\right)^{G}=\left(\left(X_{f_{k} G}\right)^{N}\right)^{G / N} \cong\left(\left(X_{f_{k} G}\right)^{N}\right)^{h_{k} G / N}=\left(R(X)^{N}\right)^{h_{k} G / N}
$$

in the $k$-local homotopy category.

Remark 7.1.5. Note that the previous proposition does not make use of the finite ved hypothesis on $G$.

As in Section 3.5, the difficulty with fully interpreting Proposition 7.1.4 as an iterated homotopy fixed point theorem is that, in general, the Quillen right derived functor of the functor

$$
(-)^{N}:\left(\Sigma \mathrm{Sp}_{G}\right)_{k} \rightarrow\left(\Sigma \mathrm{Sp}_{G / N}\right)_{k}
$$

may not agree, on the level of underlying nonequivariant spectra, with the Quillen right derived functor of the functor

$$
(-)^{N}:\left(\Sigma \mathrm{Sp}_{N}\right)_{k} \rightarrow \Sigma \mathrm{Sp}_{k}
$$

in the $k$-local homotopy category.

However, the composite

$$
\operatorname{Ho}\left(\left(\Sigma \mathrm{Sp}_{G}\right)_{k}\right) \stackrel{R(-)^{N}}{\longrightarrow} \mathrm{Ho}\left(\left(\Sigma \mathrm{Sp}_{G / N}\right)_{k}\right) \stackrel{\mathcal{U}}{\rightarrow} \operatorname{Ho}\left(\Sigma \mathrm{Sp}_{k}\right)
$$

is easily seen to be a total right derived functor (in the sense of [17, Def. 8.4.7]) of the composite

$$
\left(\Sigma \mathrm{Sp}_{G}\right)_{k} \stackrel{(-)^{N}}{\longrightarrow}\left(\Sigma \mathrm{Sp}_{G / N}\right)_{k} \stackrel{\mathcal{U}}{\rightarrow} \Sigma \mathrm{Sp}_{k} .
$$

The universal property of the total right derived functor gives a canonical natural transformation in $\operatorname{Ho}\left(\Sigma \mathrm{Sp}_{k}\right)$,

$$
\gamma_{X}: R(X)^{N} \rightarrow X^{h_{k} N}
$$

Theorem 7.1.6. The map

$$
\gamma_{E}: R(E)^{N} \rightarrow E^{h_{k} N}
$$

is an isomorphism in $\operatorname{Ho}\left(\Sigma \mathrm{Sp}_{k}\right)$.

Proof. Since $E_{f_{k} G}$ is a discrete $G$-spectrum, we have

$$
\left(E_{f_{k} G}\right)^{N}=\operatorname{colim}_{N \leq U \leq o G}\left(E_{f_{k} G}\right)^{U} .
$$

By Proposition 6.1.9, $E_{f_{k} G}$ is $k$-locally fibrant as a discrete $U$-spectrum. We therefore have a $k$-local equivalence

$$
\left(E_{f_{k} G}\right)^{N} \stackrel{\simeq_{k}}{\longrightarrow} \operatorname{colim}_{N \leq U \leq \leq_{o} G} E^{h_{k} U} .
$$

We first observe that the map

$$
\left(E_{f G}\right)^{N} \rightarrow\left(E_{f_{k} G}\right)^{N}
$$

is a $k$-local equivalence by examining the following diagram:

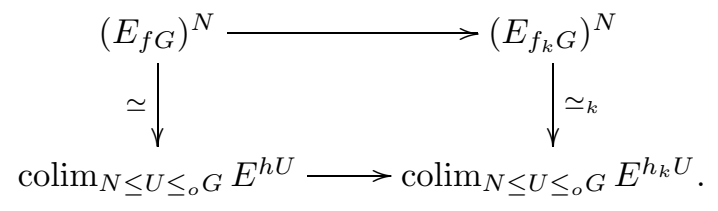


The left map is the equivalence of Corollary 3.5.2. The bottom map is a $k$-local equivalence by Proposition 6.1.7.

For a discrete $G$-spectrum $X$, the natural zig-zag

$$
X_{f_{k} G} \stackrel{\simeq_{k}}{\longrightarrow}\left(X_{f_{k} G}\right)_{f_{k} N} \stackrel{\simeq_{k}}{\longleftarrow} X_{f_{k} N}
$$

of discrete $N$-spectra induces the natural transformation

$$
\gamma_{X}: R(X)^{N}=\left(X_{f_{k} G}\right)^{N} \rightarrow X^{h_{k} N}
$$

in $\operatorname{Ho}\left(\Sigma \mathrm{Sp}_{k}\right)$. We see that $\gamma_{E}$ is a $k$-local equivalence by studying the following diagram in $\operatorname{Ho}\left(\Sigma \mathrm{Sp}_{k}\right)$ :

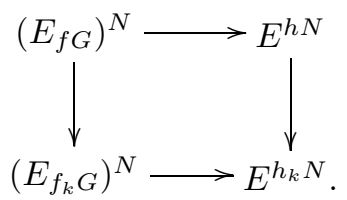

We showed that the left map is a $k$-local equivalence. The top map is a $k$-local equivalence by Corollary 7.1.3 and the right map is a $k$-local equivalence by Proposition 6.1.7.

7.2. Intermediate Galois extensions. In this subsection we will prove the forward direction of the profinite Galois correspondence.

Theorem 7.2.1. Suppose that $H$ is a closed subgroup of $G$.

(1) The spectrum $E$ is $k$-locally $H$-equivariantly equivalent to a consistent profaithful $k$-local $H$-Galois extension of $\left(E^{h H}\right)_{k}$ of finite $v c d$.

(2) If $H$ is a normal subgroup of $G$, then the spectrum $E^{h H}$ is $k$-locally $G / H$ equivariantly equivalent to a profaithful $k$-local $G / H$-Galois extension of $A$. If the quotient $G / H$ has finite $v c d$, then this extension is consistent (and of finite vcd) over $A$.

Remark 7.2.2. It is useful to note that if $G$ is a compact $p$-adic analytic group, then for any closed normal subgroup $H$ of $G$, the quotient group $G / H$ is a compact $p$-adic analytic group, and therefore must also have finite vcd [10, Thm. 9.6], [40.

Remark 7.2.3. Theorem 7.2.1, when applied to the $K(n)$-local profinite Galois extension $F_{n}$ of $S_{K(n)}$, provides an extension of [34, Thm. 5.4.4].

Proof of part (1). We shall prove that $E$ is $k$-locally $H$-equivariantly equivalent, as a discrete commutative $H$-algebra, to a commutative $\left(E^{h H}\right)_{k}$-algebra $L$. We will prove that the map

$$
\left(E^{h H}\right)_{k} \rightarrow L
$$

is a consistent profaithful $k$-local $H$-Galois extension by proving that there is a commutative diagram of commutative symmetric ring spectra

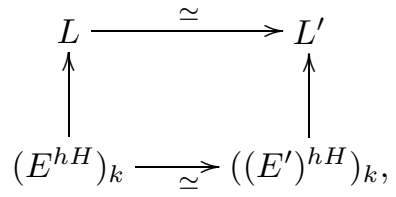

where $E^{\prime}$ is the discrete commutative $G$ - $A$-algebra, equivalent to $E$, introduced before Lemma 7.1.2, and $L^{\prime}$ is a profaithful $k$-local $H$-Galois extension of $\left(\left(E^{\prime}\right)^{h H}\right)_{k}$. The proof concludes by showing directly that $L$ is consistent over $\left(E^{h H}\right)_{k}$. 
Since $H$ is a closed subgroup of $G$, a group of finite vcd, we may conclude that $H$ has finite vcd. The system $\{H \cap V\}_{V \unlhd_{o} G}$ is cofinal in the system of open normal subgroups of $H$. Let $U=H \cap V$ be one of these open normal subgroups.

$\left(\left(E^{\prime}\right)^{h U}\right)_{k}$ is $k$-locally $H / U$-Galois over $\left(\left(E^{\prime}\right)^{h H}\right)_{k}$. We must check that the last two conditions of Definition 1.0.1 are satisfied. By Proposition 3.3.1] and Corollary 6.1.3, we have

$$
\begin{aligned}
\left(\left(\left(E^{\prime}\right)^{h U}\right)_{k}\right)^{h H / U} & \simeq\left(\left(\left(E^{\prime}\right)^{h U}\right)^{h H / U}\right)_{k} \\
& \simeq\left(\left(E^{\prime}\right)^{h H}\right)_{k}
\end{aligned}
$$

which verifies the second condition. The third condition is verified through the use of Lemma 7.1 .2 and the fact that $\left(E^{h V}\right)_{k}$ is a faithful $k$-local $H V / V$-Galois extension of $\left(E^{h H V}\right)_{k}$ (Proposition 6.3.7):

$$
\begin{aligned}
& \left(\left(\left(E^{\prime}\right)^{h U}\right)_{k} \wedge\left(\left(E^{\prime}\right)^{h H}\right)_{k}\left(\left(E^{\prime}\right)^{h U}\right)_{k}\right)_{k} \\
& \left.\quad \simeq\left(\left(\left(E^{\prime}\right)^{h H}\right)_{k} \wedge_{\left(E^{h H V}\right)_{k}}\left(E^{h V}\right)_{k}\right)_{k} \wedge\left(\left(E^{\prime}\right)^{h H}\right)_{k}\left(\left(\left(E^{\prime}\right)^{h H}\right)_{k} \wedge_{\left(E^{h H V}\right)_{k}}\left(E^{h V}\right)_{k}\right)_{k}\right)_{k} \\
& \quad \simeq\left(\left(\left(E^{\prime}\right)^{h H}\right)_{k} \wedge\left(E^{h H V}\right)_{k}\left(E^{h V}\right)_{k} \wedge\left(E^{h H V}\right)_{k}\left(E^{h V}\right)_{k}\right)_{k} \\
& \quad \simeq\left(\left(\left(E^{\prime}\right)^{h H}\right)_{k} \wedge\left(E^{h H V}\right)_{k} \operatorname{Map}\left(H V / V,\left(E^{h V}\right)_{k}\right)\right)_{k} \\
& \quad \simeq\left(\left(\left(E^{\prime}\right)^{h H}\right)_{k} \wedge_{\left(E^{h H V}\right)_{k}} \operatorname{Map}\left(H / U,\left(E^{h V}\right)_{k}\right)\right)_{k} \\
& \quad \simeq \operatorname{Map}\left(H / U,\left(\left(\left(E^{\prime}\right)^{h H}\right)_{k} \wedge_{\left(E^{h H V}\right)_{k}}\left(E^{h V}\right)_{k}\right)_{k}\right) \\
& \quad \simeq \operatorname{Map}\left(H / U,\left(\left(E^{\prime}\right)^{h U}\right)_{k}\right) .
\end{aligned}
$$

$\left(\left(E^{\prime}\right)^{h U}\right)_{k}$ is $k$-locally faithful over $\left(\left(E^{\prime}\right)^{h H}\right)_{k}$. Suppose $M$ is an $\left(\left(E^{\prime}\right)^{h H}\right)_{k^{-}}$ module and that we have

$$
\left(\left(\left(E^{\prime}\right)^{h U}\right)_{k} \wedge\left(\left(E^{\prime}\right)^{h H}\right)_{k} M\right)_{k} \simeq * .
$$

We must show $M_{k}$ is null. We use Lemma 7.1.2 to deduce

$$
\begin{aligned}
* & \simeq\left(\left(\left(E^{h V}\right)_{k} \wedge\left(E^{h H V}\right)_{k}\left(\left(E^{\prime}\right)^{h H}\right)_{k}\right)_{k} \wedge\left(\left(E^{\prime}\right)^{h H}\right)_{k} M\right)_{k} \\
& \simeq\left(\left(E^{h V}\right)_{k} \wedge\left(E^{h H V}\right)_{k} \wedge M\right)_{k} .
\end{aligned}
$$

By Proposition 6.3.7, we deduce that $\left(E^{h V}\right)_{k}$ is $k$-locally faithful over $\left(E^{h H V}\right)_{k}$, so we may conclude that $M_{k}$ is null.

Let $L$ and $L^{\prime}$ be defined by the colimits

$$
\begin{aligned}
L & :=\operatorname{colim}_{V \unlhd_{o} G}\left(E^{h H \cap V}\right)_{k}, \\
L^{\prime} & :=\operatorname{colim}_{V \unlhd_{o} G}\left(\left(E^{\prime}\right)^{h H \cap V}\right)_{k} .
\end{aligned}
$$

We have shown that the spectrum $L^{\prime}$ is a profaithful $k$-local $H$-Galois extension of $\left(\left(E^{\prime}\right)^{h H}\right)_{k}$. Furthermore, the equivalence

$$
E \rightarrow E^{\prime}
$$

of discrete commutative $G$ - $A$-algebras gives rise to Diagram (7.5).

$E$ is $k$-locally $H$-equivariantly equivalent to $L$. By Corollary 7.1 .3 , for each $V$ we have

$$
\left(\left(E_{f G}\right)^{H \cap V}\right)_{k} \simeq\left(E^{h H \cap V}\right)_{k}
$$


Since $E_{f G}$ is a discrete $H$-spectrum, we have

$$
\begin{aligned}
\left(E_{f G}\right)_{k} & =\left(\operatorname{colim}_{V \unlhd_{o} G}\left(E_{f G}\right)^{H \cap V}\right)_{k} \\
& \simeq\left(\operatorname{colim}_{V \unlhd_{o} G}\left(E^{h H \cap V}\right)_{k}\right)_{k} \\
& =L_{k} .
\end{aligned}
$$

The fibrant replacement map $E \rightarrow E_{f G}$ is an $H$-equivariant equivalence.

$L$ is consistent over $\left(E^{h H}\right)_{k}$. By Corollary 6.3.2, we just need to check that the map

$$
\left(E^{h H}\right)_{k} \rightarrow\left(L^{h H}\right)_{k}
$$

is an equivalence. We have already seen that the map $E \rightarrow L$ is a $k$-local equivalence. By Corollary 6.1.6 we see that the map of (7.6) is an equivalence.

Proof of part (2). Let $K$ be the colimit $\operatorname{colim}_{U \unlhd_{o} G}\left(E^{h H U}\right)_{k}$. Since $H$ is normal in $G$, the groups $H U$ are open normal subgroups of $G$. By Proposition 6.3.7, the spectra $\left(E^{h H U}\right)_{k}$ are $k$-local faithful $G / H U$-Galois extensions of $A$. Therefore, $K$ is a $k$-local profaithful $G / H$-Galois extension of $A$. The spectrum $K$ is $k$-locally equivalent to $E^{h H}$ by Theorem 7.1.1

Suppose that $G / H$ is of finite vcd. We are left with showing that $K$ is consistent over A. By Corollary 6.3.2, it suffices to check that the map

$$
A \rightarrow\left(K^{h G / H}\right)_{k}
$$

is an equivalence. Using Corollary 6.1.3, Theorem 7.1.1, Corollary 7.1.3, and the fact that $E$ is consistent over $A$, we have

$$
\begin{aligned}
\left(K^{h G / H}\right)_{k} & =\left(\left(\operatorname{colim}_{U \unlhd_{o} G}\left(E^{h H U}\right)_{k}\right)^{h G / H}\right)_{k} \\
& \simeq\left(\left(\operatorname{colim}_{U \unlhd_{o} G} E^{h H U}\right)^{h G / H}\right)_{k} \\
& \simeq\left(\left(\left(E_{f G}\right)^{H}\right)^{h G / H}\right)_{k} \\
& \simeq\left(E^{h G}\right)_{k} \\
& \simeq A .
\end{aligned}
$$

7.3. Function spectra. In this section, we prove the following theorem.

Theorem 7.3.1. Let $H$ and $K$ be closed subgroups of $G$. Then there is an equivalence

$$
F_{A}\left(\left(E^{h H}\right)_{k},\left(E^{h K}\right)_{k}\right) \simeq\left((E[[G / H]])^{h K}\right)_{k},
$$

where $E[[G / H]]$ has the diagonal $K$-action.

Corollary 7.3.2. If $H$ and $K$ are closed subgroups of $G$, and the left action of $K$ on $G / H$ is trivial, then there is an equivalence

$$
F_{A}\left(\left(E^{h H}\right)_{k},\left(E^{h K}\right)_{k}\right) \simeq\left(E^{h K}[[G / H]]\right)_{k} .
$$


Proof. We have the following sequence of equivalences:

$$
\begin{aligned}
F_{A}\left(\left(E^{h H}\right)_{k},\left(E^{h K}\right)_{k}\right) & \simeq\left((E[[G / H]])^{h K}\right)_{k} \\
& \simeq\left(\operatorname{holim}_{H \leq U \leq_{o} G}(E[G / U])^{h K}\right)_{k} \\
& \simeq\left(\operatorname{holim}_{H \leq U \leq o} E^{h K}[G / U]\right)_{k} \\
& \simeq\left(E^{h K}[[G / H]]\right)_{k} .
\end{aligned}
$$

Remark 7.3.3. The conclusion of Corollary 7.3.2 is typically far from true for arbitrary $H$ and $K$. For instance, let $n$ be odd. It is shown in [39, Prop. 16] that in the case of $k=K(n), A=S_{K(n)}, E=F_{n}, G=\mathbb{G}_{n}, H=\{e\}$, and $K=\mathbb{G}_{n}$, the $K(n)$-local Spanier-Whitehead dual of $E_{n}$ is given by

$$
F\left(E_{n}, E_{n}^{h \mathbb{G}_{n}}\right) \simeq F\left(E_{n}, S_{K(n)}\right) \simeq \Sigma^{-n^{2}} E_{n} \not E_{n} .
$$

The remainder of this section will be spent proving Theorem 7.3.1, We first prove some technical lemmas. Recall that an $A$-module $X$ is said to be $k$-locally $F$-small if the natural map

$$
\underset{i}{\operatorname{colim}} F_{A}\left(X, Y_{i}\right) \rightarrow F_{A}\left(X,\left(\operatorname{colim}_{i} Y_{i}\right)_{k}\right)
$$

is a $k$-local equivalence for every filtered diagram $\left\{Y_{i}\right\}_{i}$ of $k$-local $A$-modules. Observe that if $X$ is a $k$-locally dualizable $A$-module, then it is $k$-locally $F$-small, since we have

$$
\begin{aligned}
& \left(\operatorname{colim}_{i} F_{A}\left(X, Y_{i}\right)\right)_{k} \simeq\left(\operatorname{colim}_{i}\left(D_{A}(X) \wedge_{A} Y_{i}\right)_{k}\right)_{k} \\
& \simeq\left(\operatorname{colim}_{i}\left(D_{A}(X) \wedge_{A} Y_{i}\right)\right)_{k} \\
& \simeq\left(D_{A}(X) \wedge_{A} \operatorname{colim} Y_{i}\right)_{k} \\
& \simeq F_{A}\left(X,\left(\operatorname{colim} Y_{i}\right)_{k}\right) \text {. }
\end{aligned}
$$

Lemma 7.3.4. Suppose that $X$ is an A-module which is $k$-locally $F$-small, and that $Y$ is a $k$-local $A$-module. Let $T=\lim _{i} T_{i}$ be a profinite set. Then the natural map

$$
\operatorname{Map}^{c}\left(T, F_{A}(X, Y)\right) \rightarrow F_{A}\left(X, \operatorname{Map}^{c}(T, Y)_{k}\right)
$$

is a $k$-local equivalence.

Proof. We have

$$
\begin{aligned}
\operatorname{Map}^{c}\left(T, F_{A}(X, Y)\right)_{k} & =\left(\operatorname{colim}_{i} \operatorname{Map}\left(T_{i}, F_{A}(X, Y)\right)\right)_{k} \\
& \cong\left(\operatorname{colim}_{i} F_{A}\left(X, \operatorname{Map}\left(T_{i}, Y\right)\right)\right)_{k} \\
& \simeq F_{A}\left(X,\left(\operatorname{colim} \operatorname{Map}\left(T_{i}, Y\right)\right)_{k}\right) \\
& =F_{A}\left(X, \operatorname{Map}^{c}(T, Y)_{k}\right) .
\end{aligned}
$$

The following lemma is immediate from the definition of Map ${ }^{c}$. 
Lemma 7.3.5. Let $\left\{Y_{j}\right\}_{j}$ be a filtered diagram of spectra and let $T=\lim _{i} T_{i}$ be a profinite set. Then the natural map

$$
\underset{j}{\operatorname{colim}} \operatorname{Map}^{c}\left(T, Y_{j}\right) \rightarrow \operatorname{Map}^{c}\left(T, \underset{j}{\operatorname{colim}} Y_{j}\right)
$$

is an isomorphism.

Lemma 7.3.6. Let $U$ be an open subgroup of $G$, and let $V$ be an open normal subgroup of $G$, with $V \leq U$. Then there is a map of discrete $G$-A-modules

$$
\xi: A[G / U] \wedge_{A}\left(E^{h U}\right)_{k} \rightarrow\left(E^{h V}\right)_{k}
$$

where $G$ acts on the source of $\xi$ by acting only on $A[G / U]$.

Proof. To produce the map $\xi$, it suffices to construct the adjoint map of sets

$$
\widetilde{\xi}: G / U \rightarrow \operatorname{Mod}_{A}\left(\left(E^{h U}\right)_{k},\left(E^{h V}\right)_{k}\right) .
$$

Observe that for $g \in G$, the $G$-action map

$$
g: E \rightarrow E
$$

descends to a map

$$
\bar{g}: E^{h U} \rightarrow E^{h V},
$$

which localizes to give

$$
\widetilde{\xi}(g U)=(\bar{g})_{k}:\left(E^{h U}\right)_{k} \rightarrow\left(E^{h V}\right)_{k} .
$$

It is easy to check that this map is independent of the choice of coset representative. To show that $\xi$ is $G$-equivariant we must show that $\widetilde{\xi}$ is $G$-equivariant, where $G$ acts on the morphism set $\operatorname{Mod}_{A}\left(\left(E^{h U}\right)_{k},\left(E^{h V}\right)_{k}\right)$ by postcomposition. This is clear from the definition of $\widetilde{\xi}$.

The map $\xi$ gives rise to a map

$$
\psi_{V}:\left(E^{h V}\right)_{k}[G / U] \rightarrow F_{A}\left(\left(E^{h U}\right)_{k},\left(E^{h V}\right)_{k}\right)
$$

as follows: the adjoint $\widetilde{\psi}_{V}$ is given by the composite

$$
\begin{aligned}
\widetilde{\psi}_{V}:\left(E^{h V}\right)_{k} \wedge_{A}\left(A[G / U] \wedge_{A}\left(E^{h U}\right)_{k}\right) \stackrel{1 \wedge \xi}{\longrightarrow}\left(E^{h V}\right)_{k} \wedge_{A}\left(E^{h V}\right)_{k} \\
\stackrel{\mu}{\longrightarrow}\left(E^{h V}\right)_{k},
\end{aligned}
$$

where $\mu:\left(E^{h V}\right)_{k} \wedge_{A}\left(E^{h V}\right)_{k} \rightarrow\left(E^{h V}\right)_{k}$ is the multiplication map of the $A$-algebra $\left(E^{h V}\right)_{k}$. The map $\psi_{V}$ is easily checked to be $G$-equivariant, where $G$ acts diagonally on the source and acts on the target through its action on the term $\left(E^{h V}\right)_{k}$.

Lemma 7.3.7. Let $U$ be an open subgroup of $G$, and suppose that $V$ is an open normal subgroup of $G$ contained in $U$. Then the map

$$
\psi_{V}:\left(E^{h V}\right)_{k}[G / U] \stackrel{\simeq}{\rightarrow} F_{A}\left(\left(E^{h U}\right)_{k},\left(E^{h V}\right)_{k}\right)
$$

is an equivalence.

Proof. By Proposition 6.3.7, the spectrum $\left(E^{h V}\right)_{k}$ is a $k$-local $G / V$-Galois extension of $A$, and by Proposition 3.3.1, there is an equivalence

$$
E^{h U} \simeq\left(E^{h V}\right)^{h U / V} .
$$


By Proposition 6.3.5, the $A$-module $\left(E^{h V}\right)_{k}$ is $k$-locally dualizable. Making use of Corollary 6.1.3 and Lemma 6.3.4, we have

$$
\begin{aligned}
\left(\left(E^{h V}\right)_{k} \wedge_{A}\left(E^{h U}\right)_{k}\right)_{k} & \simeq\left(\left(E^{h V}\right)_{k} \wedge_{A}\left(\left(E^{h V}\right)^{h U / V}\right)_{k}\right)_{k} \\
& \simeq\left(\left(E^{h V}\right)_{k} \wedge_{A}\left(\left(E^{h V}\right)_{k}\right)^{h U / V}\right)_{k} \\
& \simeq\left(\left(\left(E^{h V}\right)_{k} \wedge_{A}\left(E^{h V}\right)_{k}\right)^{h U / V}\right)_{k} \\
& \simeq \operatorname{Map}\left(G / V,\left(E^{h V}\right)_{k}\right)^{h U / V} \\
& \simeq \operatorname{Map}\left(G / U,\left(E^{h V}\right)_{k}\right)
\end{aligned}
$$

where the last equivalence follows from the fact that the right $U / V$-action on $G / V$ is free. Applying $F_{\left(E^{h V}\right)_{k}}\left(-,\left(E^{h V}\right)_{k}\right)$ to both sides, we have

$$
\begin{aligned}
F_{A}\left(\left(E^{h U}\right)_{k},\left(E^{h V}\right)_{k}\right) & \simeq F_{\left(E^{h V}\right)_{k}}\left(\left(E^{h V}\right)_{k} \wedge_{A}\left(E^{h U}\right)_{k},\left(E^{h V}\right)_{k}\right) \\
& \simeq F_{\left(E^{h V}\right)_{k}}\left(\left(\left(E^{h V}\right)_{k} \wedge_{A}\left(E^{h U}\right)_{k}\right)_{k},\left(E^{h V}\right)_{k}\right) \\
& \simeq F_{\left(E^{h V}\right)_{k}}\left(\operatorname{Map}\left(G / U,\left(E^{h V}\right)_{k}\right),\left(E^{h V}\right)_{k}\right) \\
& \simeq F_{\left(E^{h V}\right)_{k}}\left(\left(E^{h V}\right)_{k},\left(E^{h V}\right)_{k}[G / U]\right) \\
& \simeq\left(E^{h V}\right)_{k}[G / U] .
\end{aligned}
$$

This sequence of equivalences may be checked to be compatible with the map $\psi_{V}$.

Lemma 7.3.8. Let $U$ be an open subgroup of $G$. There is an equivalence of discrete G-spectra

$$
\phi: E[G / U] \stackrel{\simeq}{\longrightarrow} \operatorname{colim}_{V \unlhd_{o} G, V \leq U} F_{A}\left(\left(E^{h U}\right)_{k},\left(E^{h V}\right)_{k}\right)
$$

Here, $G$ is acting diagonally on the left-hand side and acting only on each $\left(E^{h V}\right)_{k}$ on the right-hand side.

Remark 7.3.9. Let $V$ be an open normal subgroup of $G$ and let $U$ be an open subgroup of $G$. Since $E^{h V}$ is a $G / V$-spectrum, the function spectrum

$$
F_{A}\left(\left(E^{h U}\right)_{k},\left(E^{h V}\right)_{k}\right)
$$

is a discrete $G$-spectrum, where $G$ is acting only on the spectrum $\left(E^{h V}\right)_{k}$. The colimit

$$
\operatorname{colim}_{V \unlhd_{o} G, V \leq U} F_{A}\left(\left(E^{h U}\right)_{k},\left(E^{h V}\right)_{k}\right)
$$

is therefore a discrete $G$-spectrum.

Proof of Lemma 7.3.8. The map $\phi$ is given as the composite

$$
\begin{aligned}
E[G / U] & \stackrel{\simeq}{\rightarrow}\left(\operatorname{colim}_{V \unlhd_{o} G}\left(E^{h V}\right)_{k}\right)[G / U] \\
& \stackrel{\simeq}{\longrightarrow} \operatorname{colim}_{V \unlhd_{o} G}\left(\left(E^{h V}\right)_{k}[G / U]\right) \\
& \stackrel{\psi}{\rightarrow} \operatorname{colim}_{V \unlhd_{o} G, V \leq U} F_{A}\left(\left(E^{h U}\right)_{k},\left(E^{h V}\right)_{k}\right) .
\end{aligned}
$$

The map $\psi$ is the colimit of the equivalences

$$
\psi_{V}:\left(E^{h V}\right)_{k}[G / U] \rightarrow F_{A}\left(\left(E^{h U}\right)_{k},\left(E^{h V}\right)_{k}\right)
$$

of Lemma 7.3.7. Therefore, $\psi$ is an equivalence, so $\phi$ is an equivalence. 
Proof of Theorem 7.3.1. Fix $U$ to be an open subgroup of $G$, and suppose that $s \geq 0$. Using Lemma 7.3.8, we have

$$
\operatorname{Map}^{c}\left(K^{s}, E[G / U]\right)_{k} \simeq \operatorname{Map}^{c}\left(K^{s}, \operatorname{colim}_{V \unlhd_{o} G, V \leq U} F_{A}\left(\left(E^{h U}\right)_{k},\left(E^{h V}\right)_{k}\right)\right)_{k} .
$$

Lemma 7.3.5 gives

$$
\begin{aligned}
& \operatorname{Map}^{c}\left(K^{s}, \operatorname{colim}_{V \unlhd_{o} G, V \leq U} F_{A}\left(\left(E^{h U}\right)_{k},\left(E^{h V}\right)_{k}\right)\right)_{k} \\
& \simeq\left(\operatorname{colim}_{V \unlhd_{o} G, V \leq U} \operatorname{Map}^{c}\left(K^{s}, F_{A}\left(\left(E^{h U}\right)_{k},\left(E^{h V}\right)_{k}\right)\right)\right)_{k} .
\end{aligned}
$$

Using Lemma 7.3.4, we get

$$
\begin{aligned}
&\left(\underset{V \unlhd_{o} G, V \leq U}{\operatorname{colim}} \operatorname{Map}^{c}\left(K^{s}, F_{A}\left(\left(E^{h U}\right)_{k},\left(E^{h V}\right)_{k}\right)\right)\right)_{k} \\
& \simeq\left(\underset{V \unlhd_{o} G, V \leq U}{\operatorname{colim}} F_{A}\left(\left(E^{h U}\right)_{k}, \operatorname{Map}^{c}\left(K^{s},\left(E^{h V}\right)_{k}\right)_{k}\right)\right)_{k} .
\end{aligned}
$$

Now, by [34, Lem. 7.2.5], the spectrum $\left(E^{h U}\right)_{k}$ is a $k$-locally dualizable $A$-module. Therefore, as discussed prior to Lemma 7.3.4 it is $k$-locally $F$-small. Therefore, there is an equivalence

$$
\begin{aligned}
\left(\underset { V \unlhd _ { o } G , V \leq U } { \operatorname { c o l i m } } F _ { A } \left(\left(E^{h U}\right)_{k}, \operatorname{Map}^{c}\right.\right. & \left.\left.\left(K^{s},\left(E^{h V}\right)_{k}\right)_{k}\right)\right)_{k} \\
& \simeq F_{A}\left(\left(E^{h U}\right)_{k},\left({ }_{V \unlhd_{o} G, V \leq U}^{\operatorname{colim}_{1}} \operatorname{Map}^{c}\left(K^{s},\left(E^{h V}\right)_{k}\right)\right)_{k}\right) .
\end{aligned}
$$

Applying Lemma 7.3.5 again, we have

$$
\begin{aligned}
F_{A}\left(\left(E^{h U}\right)_{k},\left(\underset{V \unlhd_{o} G, V \leq U}{\operatorname{colim}} \operatorname{Map}^{c}\left(K^{s},\left(E^{h V}\right)_{k}\right)\right)_{k}\right) & \\
& \left.\simeq F_{A}\left(\left(E^{h U}\right)_{k}, \operatorname{Map}^{c}\left(K^{s},{ }_{V \unlhd_{o} G, V \leq U} \operatorname{colim}^{h V}\right)_{k}\right)_{k}\right) \\
& \simeq F_{A}\left(\left(E^{h U}\right)_{k}, \operatorname{Map}^{c}\left(K^{s}, E\right)_{k}\right) .
\end{aligned}
$$

We therefore have an equivalence of cosimplicial spectra

$$
\begin{aligned}
\left(\Gamma_{K}^{\bullet} E[G / U]\right)_{k} & \cong \operatorname{Map}^{c}\left(K^{\bullet}, E[G / U]\right)_{k} \\
& \simeq F_{A}\left(\left(E^{h U}\right)_{k}, \operatorname{Map}^{c}\left(K^{\bullet}, E\right)_{k}\right) \\
& \cong F_{A}\left(\left(E^{h U}\right)_{k},\left(\Gamma_{K}^{\bullet} E\right)_{k}\right) .
\end{aligned}
$$

We will now deduce the desired equivalence by producing a string of intermediate equivalences. By Theorem 4.5.1 and Corollary 6.1.3, we have

$$
\left((E[[G / H]])^{h K}\right)_{k} \simeq \operatorname{holim}_{H \leq U \leq o} \operatorname{holim}_{\Delta}\left(\Gamma_{K}^{\bullet} E[G / U]\right)_{k} .
$$

Using equivalence (7.7), we have

$$
\begin{aligned}
\operatorname{holim}_{H \leq U \leq o} \operatorname{holim}_{\Delta}\left(\Gamma_{K}^{\bullet} E[G / U]\right)_{k} & \simeq \operatorname{holim}_{H \leq U \leq o} \operatorname{holim}_{\Delta} F_{A}\left(\left(E^{h U}\right)_{k},\left(\Gamma_{K}^{\bullet} E\right)_{k}\right) \\
& \simeq \operatorname{holim}_{H \leq U \leq_{o} G} F_{A}\left(\left(E^{h U}\right)_{k}, \operatorname{holim}_{\Delta}\left(\Gamma_{K}^{\bullet} E\right)_{k}\right) .
\end{aligned}
$$

By Corollary 6.1.3, we have

$$
\operatorname{holim}_{H \leq U \leq_{o} G} F_{A}\left(\left(E^{h U}\right)_{k}, \operatorname{holim}_{\Delta}\left(\Gamma_{K}^{\bullet} E\right)_{k}\right) \simeq \operatorname{holim}_{H \leq U \leq_{o} G} F_{A}\left(\left(E^{h U}\right)_{k},\left(\operatorname{holim}_{\Delta}\left(\Gamma_{K}^{\bullet} E\right)\right)_{k}\right)
$$


Using Theorem 3.2.1 we get

$$
\begin{aligned}
\operatorname{holim}_{H \leq U \leq o} F_{A}\left(\left(E^{h U}\right)_{k},\left(\operatorname{holim}_{\Delta}\left(\Gamma_{K}^{\bullet} E\right)\right)_{k}\right) & \simeq \operatorname{holim}_{H \leq U \leq o} F_{A}\left(\left(E^{h U}\right)_{k},\left(E^{h K}\right)_{k}\right) \\
& \simeq F_{A}\left(\operatorname{colim}_{H \leq U \leq o}\left(E^{h U}\right)_{k},\left(E^{h K}\right)_{k}\right) .
\end{aligned}
$$

We may now apply Theorem 7.1.1 to deduce

$$
F_{A}\left(\operatorname{colim}_{H \leq U \leq o}\left(E^{h U}\right)_{k},\left(E^{h K}\right)_{k}\right) \simeq F_{A}\left(\left(E^{h H}\right)_{k},\left(E^{h K}\right)_{k}\right) .
$$

\section{Applications to Morava E-theory}

8.1. Morava $E$-theory as a profinite Galois extension. The general theory developed in this paper applies to Morava $E$-theory. In this setting, we have

$$
\begin{aligned}
k & =K(n), \\
A & =S_{K(n)}, \\
G & =\mathbb{G}_{n},
\end{aligned}
$$

where $K(n)$ is the $n$th Morava $K$-theory spectrum, $S_{K(n)}$ is the $K(n)$-local sphere spectrum, and $\mathbb{G}_{n}$ is the $n$th extended Morava stabilizer group:

$$
\mathbb{G}_{n}=\mathbb{S}_{n} \rtimes \operatorname{Gal}\left(\mathbb{F}_{p^{n}} / \mathbb{F}_{p}\right) .
$$

Let $E_{n}$ be the $n$th Morava $E$-theory spectrum, where

$$
\left(E_{n}\right)_{*}=W\left(\mathbb{F}_{p^{n}}\right)\left[\left[u_{1}, \ldots, u_{n-1}\right]\right]\left[u^{ \pm 1}\right] .
$$

Here, the degree of $u$ is -2 and the complete power series ring is in degree zero. Goerss and Hopkins [16, building on work of Hopkins and Miller [33], showed that $\mathbb{G}_{n}$ acts on $E_{n}$ by maps of commutative $S$-algebras.

Devinatz and Hopkins [9] constructed homotopy fixed point spectra

$$
E_{n}^{d h H}
$$

for closed subgroups $H$ of $\mathbb{G}_{n}$. (Here we use the notation $E_{n}^{d h H}$ to distinguish the Devinatz-Hopkins homotopy fixed point spectra from the homotopy fixed point spectra constructed in this paper.) We give a brief overview of the way that Devinatz and Hopkins defined $E_{n}^{d h H}$.

(1) For $U$ an open subgroup of $\mathbb{G}_{n}$, Devinatz and Hopkins form a cosimplicial object in the stable homotopy category

$$
\widetilde{\mathbf{C}}_{U}^{\bullet}: \Delta \rightarrow \operatorname{Ho}(\Sigma \mathrm{Sp})
$$

having the property that

$$
\widetilde{\mathbf{C}}_{U}^{s}=\left(E_{n}^{\wedge s} \wedge \operatorname{Map}\left(\mathbb{G}_{n} / U, E_{n}\right)\right)_{K(n)} .
$$

This would yield a $K(n)$-local $E_{n}$-based Adams resolution of $E_{n}^{d h U}$, if this spectrum were to exist, given that one expects

$$
\left(E_{n} \wedge E_{n}^{d h U}\right)_{K(n)} \simeq \operatorname{Map}\left(\mathbb{G}_{n} / U, E_{n}\right) .
$$

The cosimplicial objects taken together give a functor

$$
\widetilde{\mathbf{C}}_{(-)}^{\bullet}:\left(\mathcal{O}_{\mathbb{G}_{n}}^{\text {fin }}\right)^{\mathrm{op}} \rightarrow c \mathrm{Ho}(\Sigma \mathrm{Sp})
$$


from the opposite category of finite $\mathbb{G}_{n}$-orbits to the category of cosimplicial objects in the stable homotopy category.

(2) Using the $E_{\infty}$-mapping space calculations of [16], together with the rectification machinery of [1], Devinatz and Hopkins show that the diagram $\widetilde{\mathbf{C}}_{U}^{\bullet}$ admits a rectification to give a point-set level commutative diagram

$$
\mathbf{C}_{U}^{\bullet}: \Delta \rightarrow \operatorname{Alg}_{S} .
$$

Furthermore, they show that the rectification can be made functorial in $U$.

(3) The spectrum $E_{n}^{d h U}$ is defined by

$$
E_{n}^{d h U}:=\underset{\Delta}{\operatorname{holim}} \mathbf{C}_{U}^{\bullet}
$$

When $U$ is normal in $\mathbb{G}_{n}$, the functoriality of $\mathbf{C}_{U}^{\bullet}$ in $U$ induces a $\mathbb{G}_{n} / U$ action on $E_{n}^{d h U}$ by maps of $S$-algebras.

(4) For $H$ a closed subgroup of $\mathbb{G}_{n}$, the spectrum $E_{n}^{d h H}$ is defined by

$$
E_{n}^{d h H}:=\left(\operatorname{colim}_{H \leq U \leq \mathbb{G}_{n}} E_{n}^{d h U}\right)_{K(n)} .
$$

Rognes [34, Thm. 5.4.4, Lem. 4.3.7] observed, for $U$ an open normal subgroup of $\mathbb{G}_{n}$, that the work of Devinatz and Hopkins [8, 9] proves that $E_{n}^{d h U}$ is a faithful $K(n)$-local $\mathbb{G}_{n} / U$-Galois extension of $S_{K(n)}$. Therefore, the discrete commutative $\mathbb{G}_{n}-S_{K(n)}$-algebra

$$
F_{n}=\underset{U \unlhd_{o} \mathbb{G}_{n}}{\operatorname{colim}} E_{n}^{d h U}
$$

is a profaithful $K(n)$-local profinite $\mathbb{G}_{n}$-Galois extension of $S_{K(n)}$. The spectrum $E_{n}$ is recovered by the equivalence (see [9, Def. 1.5, Thm. 3(i)])

$$
E_{n} \simeq\left(F_{n}\right)_{K(n)} .
$$

With this in mind, we make the following definition.

Definition 8.1.1. For $H$ a closed subgroup of $\mathbb{G}_{n}$, we define

$$
E_{n}^{h H}:=\left(F_{n}^{h H}\right)_{K(n)},
$$

which is a commutative $S_{K(n)}$-algebra by Lemma 5.2.6,

We note that the use of pro-spectra gives an alternative, but equivalent approach to defining $E_{n}^{h H}$. For a sequence of integers $I=\left(i_{0}, \ldots, i_{n-1}\right)$, we shall let $M_{I}$ denote the generalized Moore spectrum with

$$
B P_{*} M_{I} \cong B P_{*} /\left(p^{i_{0}}, v_{1}^{i_{1}}, \ldots, v_{n-1}^{i_{n-1}}\right) .
$$

These spectra are inductively defined by cofiber sequences

$$
\Sigma^{2 i_{j}\left(p^{j}-1\right)} M_{\left(i_{0}, \ldots, i_{j-1}\right)} \stackrel{v_{j}^{i_{j}}}{\longrightarrow} M_{\left(i_{0}, \ldots, i_{j-1}\right)} \rightarrow M_{\left(i_{0}, \ldots, i_{j}\right)},
$$

where $v_{j}^{i_{j}}$ is a $v_{j}$ self-map. While $M_{I}$ does not exist for every $I$, the Hopkins-Smith Periodicity Theorem implies that the maps $v_{j}^{i_{j}}$ exist for $i_{j} \gg 1$.

As explained in [22, Prop. 7.10], one can form a pro-spectrum $\left\{M_{I}\right\}_{I}$ for a cofinal system of multi-indices $I$. Then the pro-spectrum

$$
\mathbf{E}_{n}=\left\{F_{n} \wedge M_{I}\right\}_{I}
$$


is a continuous $H$-spectrum. Since $F_{n}$ is $E(n)$-local and each $M_{I}$ is a finite spectrum, the homotopy fixed points are identified by

$$
\begin{aligned}
E_{n}^{h H} & =\underset{I}{\operatorname{holim}}\left(F_{n} \wedge M_{I}\right)^{h H} \\
& \simeq \operatorname{holim}_{I} F_{n}^{h H} \wedge M_{I} \\
& \simeq\left(F_{n}^{h H}\right)_{K(n)} .
\end{aligned}
$$

Thus, the homotopy fixed points of the continuous $H$-spectrum $\mathbf{E}_{n}$ coincide with the $K(n)$-localization of the homotopy fixed points of the discrete $H$-spectrum $F_{n}$. In particular, Definition 8.1.1 is equivalent to the definition of $E_{n}^{h H}$ given in 6].

Proposition 8.1.2. The profaithful $K(n)$-local profinite $\mathbb{G}_{n}$-Galois extension $F_{n}$ of $S_{K(n)}$ is consistent and has finite vcd.

Proof. There is a zig-zag

$$
\left(F_{n}^{\wedge S_{K(n)}}\right)_{K(n)} \stackrel{\simeq}{\longleftarrow}\left(F_{n}^{\wedge \bullet+1}\right)_{K(n)} \stackrel{\simeq}{\rightarrow}\left(\left(F_{n}\right)_{K(n)}^{\wedge \bullet+1}\right)_{K(n)} \simeq\left(E_{n}^{\wedge \bullet+1}\right)_{K(n)}
$$

of levelwise equivalences of cosimplicial objects. We therefore have the equivalences

$$
\begin{aligned}
\left(S_{K(n)}\right)_{K(n), F_{n}}^{\wedge} & =\underset{\Delta}{\operatorname{holim}}\left({F_{n}^{\wedge S_{K(n)}}}^{\bullet+1}\right)_{K(n)} \\
& \simeq \operatorname{holim}_{\Delta}\left(E_{n}^{\wedge \bullet+1}\right)_{K(n)} \\
& \simeq S_{K(n)},
\end{aligned}
$$

where the last equivalence follows from the fact that the cosimplicial object

$$
\left(E_{n}^{\wedge \bullet+1}\right)_{K(n)}
$$

is the $K(n)$-local $E_{n}$-Adams resolution for $S_{K(n)}$.

Since $\mathbb{G}_{n}$ is a compact $p$-adic analytic group, it has finite virtual cohomological dimension. Therefore, the extension $F_{n}$ of $S_{K(n)}$ has finite vcd.

Corollary 8.1.3. There is a weak equivalence $E_{n}^{h \mathbb{G}_{n}} \simeq S_{K(n)}$.

Proof. This follows immediately from Corollary 6.3.2.

Remark 8.1.4. Because of the result of Devinatz and Hopkins that there is an equivalence $E_{n}^{d h \mathbb{G}_{n}} \simeq S_{K(n)}$ [9, Theorem 1(iii)], it has been known for some time that the $K(n)$-local sphere behaves like a homotopy fixed point spectrum; Corollary 8.1 .3 makes this idea precise.

8.2. Comparison with the Devinatz-Hopkins homotopy fixed points. Let $H$ be a closed subgroup of $\mathbb{G}_{n}$. The following theorem relates the homotopy fixed point construction $E_{n}^{h H}$ of Definition 8.1.1 to the Devinatz-Hopkins homotopy fixed point construction $E_{n}^{d h H}$ of $[9$.

Theorem 8.2.1. If $H$ is a closed subgroup of $\mathbb{G}_{n}$, there is an equivalence

$$
E_{n}^{d h H} \simeq E_{n}^{h H} .
$$

Proof. As explained in Subsection 8.1 $F_{n}=\operatorname{colim}_{U \unlhd_{o} \mathbb{G}_{n}} E_{n}^{d h U}$ is a consistent profaithful $K(n)$-local profinite $\mathbb{G}_{n}$-Galois extension of $S_{K(n)}$ of finite vcd. Thus, by Lemma 6.3.6, for each $U \unlhd_{o} \mathbb{G}_{n}$, there is a $\mathbb{G}_{n} / U$-equivariant equivalence

$$
E_{n}^{d h U} \simeq\left(F_{n}^{h U}\right)_{K(n)} .
$$


Therefore, given a generalized Moore spectrum $M_{I}$, there is an equivalence

$$
E_{n}^{d h U} \wedge M_{I} \simeq F_{n}^{h U} \wedge M_{I} .
$$

By Theorem 7.1.1 the natural map

$$
\left(\operatorname{colim}_{H \leq V \leq o} F_{n}^{h V}\right)_{K(n)} \rightarrow\left(F_{n}^{h H}\right)_{K(n)}
$$

is an equivalence.

Let $V$ be any open subgroup of $\mathbb{G}_{n}$. Then $V$ contains a subgroup $W$ such that $W$ is an open normal subgroup of $\mathbb{G}_{n}$. We have the following chain of equivalences:

$$
\begin{aligned}
E_{n}^{d h V} \wedge M_{I} & \simeq\left(E_{n}^{d h W}\right)^{h V / W} \wedge M_{I} \simeq\left(E_{n}^{d h W} \wedge M_{I}\right)^{h V / W} \\
& \simeq\left(F_{n}^{h W} \wedge M_{I}\right)^{h V / W} \simeq\left(F_{n}^{h W}\right)^{h V / W} \wedge M_{I} \\
& \simeq F_{n}^{h V} \wedge M_{I},
\end{aligned}
$$

where the first equivalence is [9, Thm. 4], the second and fourth equivalences follow from the fact that $M_{I}$ is a finite spectrum, the third equivalence is because of (8.1), and the last equivalence is due to Proposition 3.3 .1

Recall from Definition 8.1.1 that $E_{n}^{h H}=\left(F_{n}^{h H}\right)_{K(n)}$. Then the above observations imply that

$$
\begin{aligned}
E_{n}^{h H} & \simeq\left(\operatorname{colim}_{H \leq V \leq o} F_{n} F_{n}^{h V}\right)_{K(n)} \\
\simeq \operatorname{holim}_{I} \operatorname{colim}_{H \leq V \leq o}\left(\mathbb{G}_{n}\right. & \left(F_{n}^{h V} \wedge M_{I}\right) \\
& \simeq \operatorname{holim}_{I} \operatorname{colim}_{H \leq V \leq_{o} \mathbb{G}_{n}}\left(E_{n}^{d h V} \wedge M_{I}\right) \\
& \simeq\left(\operatorname{colim}_{H \leq V \leq \mathbb{G}_{n}} E_{n}^{d h V}\right)_{K(n)} \\
& \simeq E_{n}^{d h H}
\end{aligned}
$$

where the last equivalence follows from [9, Def. 1.5].

Remark 8.2.2. As mentioned in the Introduction, Theorem 8.2.1 first appeared in the second author's thesis [5]. The arguments in [5] relied on a somewhat complicated analysis of a $K(n)$-local $E_{n}$-Adams resolution of $E_{n}^{d h H}$, whereas our proof makes use of Rognes's Galois theory and, consequently, is more efficient.

Corollary 8.2.3. If $H$ is a closed subgroup of $\mathbb{G}_{n}$ and $X$ is a finite spectrum, then there is an equivalence

$$
E_{n}^{d h H} \wedge X \simeq\left(E_{n} \wedge X\right)^{h H} .
$$

Proof. By [6, Thm. 1.3, Rmk. 9.3], $E_{n} \wedge X$ is a continuous $H$-spectrum (in the sense of [6]). Then, by Theorem 8.2.1 and [6. Thm. 9.9],

$$
E_{n}^{d h H} \wedge X \simeq E_{n}^{h H} \wedge X \simeq\left(E_{n} \wedge X\right)^{h H} .
$$

Corollary 8.2.4. If $X$ is a finite spectrum, then there is an equivalence

$$
X_{K(n)} \simeq\left(E_{n} \wedge X\right)^{h \mathbb{G}_{n}} .
$$

Proof. By [6, Thm. 9.9] and Corollary 8.1 .3 ,

$$
\left(E_{n} \wedge X\right)^{h \mathbb{G}_{n}} \simeq E_{n}^{h \mathbb{G}_{n}} \wedge X \simeq S_{K(n)} \wedge X \simeq X_{K(n)},
$$

where the last equivalence follows from the fact that $X$ is finite. 
Let $X$ be a finite spectrum. By [9, Thm. 2(ii)], there is a strongly convergent $K(n)$-local $E_{n}$-Adams spectral sequence that has the form

$$
H_{c}^{s}\left(H ; \pi_{t}\left(E_{n} \wedge X\right)\right) \Rightarrow \pi_{t-s}\left(E_{n}^{d h H} \wedge X\right) .
$$

Also, by [6, Thm. 1.7], there is a descent spectral sequence

$$
H_{c}^{s}\left(H ; \pi_{t}\left(E_{n} \wedge X\right)\right) \Rightarrow \pi_{t-s}\left(\left(E_{n} \wedge X\right)^{h H}\right) .
$$

Theorem 8.2.5. If $H$ is a closed subgroup of $\mathbb{G}_{n}$ and $X$ is a finite spectrum, then the spectral sequence (8.2) is isomorphic to the spectral sequence (8.3) from the $E_{2}$-terms onward.

Proof. By [18, proof of Prop. 7.4], the spectral sequence [8.2) is the inverse limit over $\{I\}$ of $K(n)$-local $E_{n}$-Adams spectral sequences that have the form

$$
{ }^{I} E_{2}^{s, t}(I)=H_{c}^{s}\left(H ; \pi_{t}\left(E_{n} \wedge M_{I} \wedge X\right)\right) \Rightarrow \pi_{t-s}\left(E_{n}^{d h H} \wedge M_{I} \wedge X\right) .
$$

Similarly, the spectral sequence (8.3) is the inverse limit over $\{I\}$ of conditionally convergent descent spectral sequences that have the form

$$
{ }^{I I} E_{2}^{s, t}(I)=H_{c}^{s}\left(H ; \pi_{t}\left(E_{n} \wedge M_{I} \wedge X\right)\right) \Rightarrow \pi_{t-s}\left(E_{n}^{h H} \wedge M_{I} \wedge X\right) .
$$

Henceforth, we write the $E_{2}$-terms ${ }^{I} E_{2}^{s, t}(I)$ and ${ }^{I I} E_{2}^{s, t}(I)$ as ${ }^{I} E_{2}^{s, t}$ and ${ }^{I I} E_{2}^{s, t}$, respectively.

Note that the spectral sequence (8.4) is isomorphic to the strongly convergent $K(n)$-local $E_{n}$-Adams spectral sequence

$$
{ }^{I} E_{2}^{s, t} \cong H_{c}^{s}\left(H ;\left(E_{n}\right)^{-t}\left(D X \wedge D M_{I}\right)\right) \Rightarrow\left(E_{n}^{d h H}\right)^{-t+s}\left(D X \wedge D M_{I}\right) .
$$

Thus, to prove the theorem, it suffices to show that the spectral sequences in (8.5) and (8.6) are isomorphic to each other.

Notice that

$$
\begin{aligned}
& { }^{I} E_{2}^{s, t} \cong{ }^{I I} E_{2}^{s, t} \\
& \cong \operatorname{colim}_{N \unlhd_{0} \mathbb{G}_{n}} H^{s}\left(H /(H \cap N) ; \pi_{t}\left(E_{n}^{d h N} \wedge M_{I} \wedge X\right)\right) \\
& \cong \operatorname{colim}_{N \unlhd_{o} \mathbb{G}_{n}} H^{s}\left(N H / N ;\left(E_{n}^{d h N}\right)^{-t}\left(D X \wedge D M_{I}\right)\right) \\
& =\operatorname{colim}_{N \unlhd_{o} \mathbb{G}_{n}}{ }^{I I I} E_{2}^{s, t}(N),
\end{aligned}
$$

where ${ }^{I I I} E_{2}^{s, t}(N)$ is the $E_{2}$-term of the strongly convergent spectral sequence ${ }^{I I I} E_{r}^{*, *}(N)$, which has the form

$$
H^{s}\left(N H / N ;\left(E_{n}^{d h N}\right)^{-t}\left(D X \wedge D M_{I}\right)\right) \Rightarrow\left(E_{n}^{d h N H}\right)^{-t+s}\left(D X \wedge D M_{I}\right)
$$

and is the Adams spectral sequence constructed by Devinatz in [8, (0.1)].

By Lemma 8.2.7 below, there is a map from the spectral sequence (8.7) to the spectral sequence (8.6), such that the isomorphism

$$
{ }^{I} E_{2}^{s, t} \cong \operatorname{colim}_{N \unlhd_{o} \mathbb{G}_{n}}{ }^{I I I} E_{2}^{s, t}(N)
$$

implies that the spectral sequence of (8.6) is isomorphic to the spectral sequence $\operatorname{colim}_{N \unlhd_{o} \mathbb{G}_{n}}{ }^{I I I} E_{r}^{*, *}(N)$. Thus, we only have to show that the spectral sequences (8.5) and $\operatorname{colim}_{N \unlhd_{0} \mathbb{G}_{n}}{ }^{I I I} E_{r}^{*, *}(N)$ are isomorphic to each other. 
By [8, Thm. A.1], ${ }^{I I} E_{r}^{*, *}(N)$ is isomorphic to the usual descent spectral sequence ${ }^{I V} E_{r}^{*, *}(N)$ that has the form

$$
H^{s}\left(N H / N ;\left(E_{n}^{d h N}\right)^{-t}\left(D X \wedge D M_{I}\right)\right) \Rightarrow\left(\left(E_{n}^{d h N}\right)^{h N H / N}\right)^{-t+s}\left(D X \wedge D M_{I}\right),
$$

since, in the notation of [8, App. A], the "homotopy fixed point spectral sequence" with abutment

$$
\left[E_{n}^{d h N H} \wedge D X \wedge D M_{I},\left(E_{n}^{d h N}\right)^{h N H / N}\right]_{E_{n}^{d h N H}}^{*}
$$

which is isomorphic to $\left[D X \wedge D M_{I},\left(E_{n}^{d h N}\right)^{h N H / N}\right]^{*}$, is equivalent to ${ }^{I V} E_{r}^{*, *}(N)$. Because of the isomorphism

$$
\operatorname{colim}_{N \unlhd_{o} \mathbb{G}_{n}}{ }^{I I I} E_{r}^{*, *}(N) \cong \operatorname{colim}_{N \unlhd_{o} \mathbb{G}_{n}} I V E_{r}^{*, *}(N),
$$

our proof reduces to showing that (8.5) and $\operatorname{colim}_{N \unlhd_{o} \mathbb{G}_{n}} I V E_{r}^{*, *}(N)$ are isomorphic spectral sequences.

The abutment of the spectral sequence (8.5) is the homotopy of

$$
\begin{aligned}
& E_{n}^{h H} \wedge M_{I} \wedge X \simeq\left(F_{n} \wedge M_{I} \wedge X\right)^{h H} \\
& \cong \underset{\Delta}{\operatorname{holim}} \operatorname{Map}^{c}\left(H^{\bullet}, \underset{N \unlhd_{o} \mathbb{G}_{n}}{\operatorname{colim}}\left(E_{n}^{d h N} \wedge M_{I} \wedge X\right)\right) \\
& \cong \operatorname{holim} \underset{N}{\operatorname{colim}} \operatorname{Map}_{o} \operatorname{Map}^{c}\left((N H / N)^{\bullet}, E_{n}^{d h N} \wedge M_{I} \wedge X\right) \text {, }
\end{aligned}
$$

where the first equivalence is by [6, Cor. 9.8] and the second equivalence is an identification, given by Theorem 3.2.1. Since $N H / N$ is a finite group, there is an identification

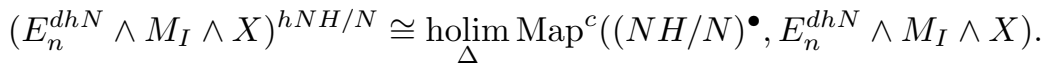

Thus, for each $U \unlhd_{o} \mathbb{G}_{n}$, the canonical map

$$
\operatorname{Map}^{c}\left((U H / U)^{\bullet}, E_{n}^{d h U} \wedge M_{I} \wedge X\right) \rightarrow \operatorname{colim}_{N \unlhd_{o} \mathbb{G}_{n}} \operatorname{Map}^{c}\left((N H / N)^{\bullet}, E_{n}^{d h N} \wedge M_{I} \wedge X\right)
$$

of cosimplicial spectra induces a map

$$
\left(E_{n}^{d h U} \wedge M_{I} \wedge X\right)^{h U H / U} \rightarrow\left(F_{n} \wedge M_{I} \wedge X\right)^{h H}
$$

and a map

$$
\psi_{U}:{ }^{V} E_{r}^{*, *}(U) \rightarrow{ }^{I I} E_{r}^{*, *}
$$

of conditionally convergent spectral sequences, where ${ }^{V} E_{r}^{*, *}(U)$ is the descent spectral sequence that has the form

$$
H^{s}\left(U H / U ; \pi_{t}\left(E_{n}^{d h U} \wedge M_{I} \wedge X\right)\right) \Rightarrow \pi_{t-s}\left(\left(E_{n}^{d h U} \wedge M_{I} \wedge X\right)^{h U H / U}\right) .
$$

It will be helpful to note that the abutment of ${ }^{V} E_{r}^{*, *}(U)$ can also be written as $\pi_{t-s}\left(\left(E_{n}^{d h U}\right)^{h U H / U} \wedge M_{I} \wedge X\right)$.

Since the map $\operatorname{colim}_{N \unlhd_{o} \mathbb{G}_{n}} \psi_{N}$ induces an isomorphism

$$
\operatorname{colim}_{N \unlhd_{o} \mathbb{G}_{n}}{ }^{V} E_{2}^{s, t}(N) \cong{ }^{I I} E_{2}^{s, t},
$$

there is an isomorphism between the spectral sequences colim $\operatorname{s\unlhd }_{o} \mathbb{G}_{n} V E_{r}^{*, *}(N)$ and (8.5). Therefore, the proof is completed by showing that there is an isomorphism

$$
\operatorname{colim}_{N \unlhd_{o} \mathbb{G}_{n}} I V E_{r}^{*, *}(N) \cong \operatorname{colim}_{N \unlhd_{o} \mathbb{G}_{n}} V E_{r}^{*, *}(N)
$$

of spectral sequences; this isomorphism follows from the fact that the spectral sequences ${ }^{I V} E_{r}^{*, *}(N)$ and ${ }^{V} E_{r}^{*, *}(N)$ are equivalent to each other. 
The following results are needed for the above proof.

Lemma 8.2.6. Suppose that $A$ is a $k$-local commutative symmetric ring spectrum and that $E$ is a k-local commutative A-algebra. Then the canonical $k$-local $E$ resolution of $A$ in the category of $A$-modules

$$
* \rightarrow A \rightarrow E \rightarrow\left(E \wedge_{A} E\right)_{k} \rightarrow\left(E \wedge_{A} E \wedge_{A} E\right)_{k} \rightarrow \cdots
$$

is a $k$-local E-resolution of $A$ in the category of $S$-modules.

Proof. We use the terminology of [31, adapted to the category of $k$-local $A$-modules as in [8, Sec. 2]. To prove the lemma, we will show that the associated $k$-local $E$ Adams resolution of $A$-modules

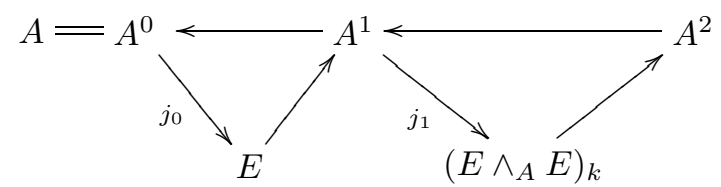

is a $k$-local $E$-Adams resolution of $S$-modules. It suffices to verify that $(1)$ for all $s>0$, the spectra $\left(E^{\wedge} A^{S}\right)_{k}$ are $k$-local $E$-injective, and (2) that each of the maps $j_{i}$ in 8.8 is $k$-local $E$-monic.

Claim (1) follows from the fact that the map

$$
\left(E^{\wedge A_{A} s}\right)_{k}=\left(S \wedge E^{\wedge_{A} s}\right)_{k} \rightarrow\left(E \wedge E^{\wedge_{A} s}\right)_{k}
$$

is split-monic. Claim (2) follows from the fact that every $k$-local $E$-monic map of $A$-modules is $k$-local $E$-monic as a map of $S$-modules. Indeed, if $f: X \rightarrow Y$ is a $k$-local $E$-monic map of $A$-modules, then consider the following diagram:

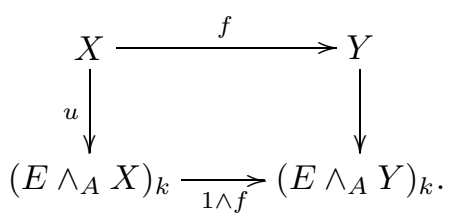

The map $u$ is seen to be a $k$-local $E$-monic map of $S$-modules because the map

$$
(E \wedge X)_{k} \stackrel{1 \wedge u}{\longrightarrow}(E \wedge E \wedge A X)_{k}
$$

is split-monic. Because $f$ is a $k$-local $E$-monic map of $A$-modules, the map $1 \wedge f$ is split-monic, and therefore $1 \wedge f$ is a $k$-local $E$-monic map of $S$-modules. We deduce that $f$ is a $k$-local $E$-monic map of $S$-modules.

Lemma 8.2.7. Let $H$ be a closed subgroup of $\mathbb{G}_{n}$ and let $N$ be an open normal subgroup. Then, for any spectrum $Z$, there is a natural map from the Adams spectral sequence

$$
H^{s}\left(N H / N ;\left(E_{n}^{d h N}\right)^{-t}(Z)\right) \Rightarrow\left(E_{n}^{d h N H}\right)^{-t+s}(Z)
$$

of [8, (0.1)] to the Adams spectral sequence

$$
H_{c}^{s}\left(H ;\left(E_{n}\right)^{-t}(Z)\right) \Rightarrow\left(E_{n}^{d h H}\right)^{-t+s}(Z)
$$

of [9, Thm. 2(ii)]. When $Z=D M_{I} \wedge Z^{\prime}$, where $Z^{\prime}$ is any finite spectrum, the induced map on $E_{2}$-terms is the usual map in continuous group cohomology that is induced by the canonical maps $H \rightarrow N H / N$ and $E_{n}^{d h N} \rightarrow \operatorname{colim}_{U \unlhd_{o} \mathbb{G}_{n}} E_{n}^{d h U}$. 
Proof. To ease our notation, we write $E_{n}^{h K}$ in place of $E_{n}^{d h K}$, whenever $K$ is closed in $\mathbb{G}_{n}$. Also, we take implicit cofibrant replacements as needed. The first spectral sequence is formed from the resolution

$$
* \rightarrow E_{n}^{h N H} \rightarrow E_{n}^{h N} \rightarrow\left(E_{n}^{h N} \wedge_{E_{n}^{h N H}} E_{n}^{h N}\right)_{K(n)} \rightarrow \cdots
$$

(see the discussions after [8, (0.1) and Prop. 3.6]). By Lemma 8.2.6, the canonical $K(n)$-local $E_{n}$-resolution

$$
* \rightarrow E_{n}^{h H} \rightarrow E_{n} \rightarrow\left(E_{n} \wedge_{E_{n}^{h H}} E_{n}\right)_{K(n)} \rightarrow\left(E_{n} \wedge_{E_{n}^{h H}} E_{n} \wedge_{E_{n}^{h H}} E_{n}\right)_{K(n)} \rightarrow \cdots
$$

of $E_{n}^{h H}$ in the category of $E_{n}^{h H}$-modules is also a $K(n)$-local $E_{n}$-resolution of $E_{n}^{h H}$ in the category of $S$-modules. Thus, the second spectral sequence, which was originally constructed by using such a resolution in the category of $S$-modules (see [9. p. 32 , App. A]), can be regarded as a $K(n)$-local $E_{n}$-Adams spectral sequence in the category of $E_{n}^{h H}$-modules, so that we can also regard the second spectral sequence as being given by [8, (0.1)] through the resolution

$$
* \rightarrow E_{n}^{h H} \rightarrow E_{n} \rightarrow\left(E_{n} \wedge_{E_{n}^{h H}} E_{n}\right)_{K(n)} \rightarrow\left(E_{n} \wedge_{E_{n}^{h H}} E_{n} \wedge_{E_{n}^{h H}} E_{n}\right)_{K(n)} \rightarrow \cdots
$$

As in [8, (3.7)], there is a canonical map to the preceding resolution, from the resolution

$$
\left(\underset{U \unlhd_{o} \mathbb{G}_{n}}{\operatorname{colim}} E_{n}^{h U H}\right)_{K(n)} \rightarrow\left(\underset{U \unlhd_{o} \mathbb{G}_{n}}{\operatorname{colim}} E_{n}^{h U}\right)_{K(n)} \rightarrow\left(\underset{U \unlhd_{o} \mathbb{G}_{n}}{\operatorname{colim}}\left(E_{n}^{h U} \wedge_{E_{n}^{h U H}} E_{n}^{h U}\right)\right)_{K(n)} \rightarrow \cdots,
$$

and this map is a levelwise weak equivalence (at the beginning of the last resolution, the usual " $* \rightarrow$ " was omitted for the sake of space). This last resolution receives the obvious map from the first resolution

$$
* \rightarrow E_{n}^{h N H} \rightarrow E_{n}^{h N} \rightarrow\left(E_{n}^{h N} \wedge_{E_{n}^{h N H}} E_{n}^{h N}\right)_{K(n)} \rightarrow \cdots .
$$

Thus, composition gives a map $\lambda$ from the resolution for the first spectral sequence to the resolution for the second spectral sequence; $\lambda$ induces the desired map of spectral sequences.

By [8, Cor. 3.9],

$$
\pi_{*}\left(\left(E_{n}^{h N} \wedge_{E_{n}^{h N H}} E_{n}^{h N}\right)_{K(n)}\right) \cong \operatorname{Map}^{c}\left(N H / N, \pi_{*}\left(E_{n}^{h N}\right)\right)
$$

and

$$
\pi_{*}\left(\left(E_{n} \wedge_{E_{n}^{h H}} E_{n}\right)_{K(n)}\right) \cong \operatorname{Map}^{c}\left(H, \pi_{*}\left(E_{n}\right)\right),
$$

and, hence, the last statement of the lemma follows easily from the definition of $\lambda$ and [8, proof of Thm. 3.1].

\section{ACKNOWLEDGMENTS}

The first author benefitted from the input of Halvard Fausk, Paul Goerss, and Daniel Isaksen. The second author thanks Paul Goerss for helpful discussions, when he was a Ph.D. student, regarding the results in Sections 8.1 and 8.2 Also, the second author is grateful to Paul for later helpful conversations, to Mark Hovey for providing some intuition related to Theorem 2.3.2, and to John Rognes for a helpful discussion regarding group actions. The second author spent several weeks in the summer of 2008 in the Department of Math at Rice University working on this paper, and he thanks the department for its hospitality. The authors would also like to express their thanks to the referee, for suggesting numerous improvements to this paper. 


\section{REFERENCES}

[1] A. Baker, B. Richter. Realizability of algebraic Galois extensions by strictly commutative ring spectra. Trans. Amer. Math. Soc. 359 (2007), no. 2, 827-857. MR2255198 (2007m:55007)

[2] M. Behrens, A modular description of the $K(2)$-local sphere at the prime 3 . Topology 45 (2006), no. 2, 343-402. MR2193339 (2006i:55016)

[3] M. Behrens, T. Lawson, Topological Automorphic Forms. Mem. Amer. Math. Soc. 204 (2010), no. 958 , xxiii $+136 \mathrm{pp}$.

[4] A.K. Bousfield, The localization of spectra with respect to homology. Topology 18 (1979), no. 4, 257-281. MR.551009 (80m:55006)

[5] D.G. Davis, The Lubin-Tate spectrum and its homotopy fixed point spectra. Thesis, Northwestern University, 2003.

[6] D.G. Davis, Homotopy fixed points for $L_{K(n)}\left(E_{n} \wedge X\right)$ using the continuous action. J. Pure Appl. Algebra 206 (2006), no. 3, 322-354. MR2235364 (2007b:55008)

[7] D.G. Davis, Iterated homotopy fixed points for the Lubin-Tate spectrum. With an appendix by D. G. Davis and B. Wieland. Topology Appl. 156 (2009), no. 17, 2881-2898. MR2556043

[8] E.S. Devinatz, A Lyndon-Hochschild-Serre spectral sequence for certain homotopy fixed point spectra. Trans. Amer. Math. Soc. 357 (2005), 129-150. MR2098089 (2006b:55007)

[9] E.S. Devinatz, M.J. Hopkins, Homotopy fixed point spectra for closed subgroups of the Morava stabilizer groups. Topology 43 (2004), no. 1, 1-47. MR2030586 (2004i:55012)

[10] J.D. Dixon, M.P.F. du Sautoy, A. Mann, D. Segal, Analytic pro- $p$ groups. Cambridge University Press, Cambridge, second edition, 1999. MR.1720368 (2000m:20039)

[11] W.G. Dwyer, D.M. Kan, J.H. Smith, Homotopy commutative diagrams and their realizations. J. Pure Appl. Algebra 57 (1989), no. 1, 5-24. MR984042 (90d:18007)

[12] H. Fausk, Equivariant homotopy theory for pro-spectra. Geometry and Topology 12 (2008), no. 1, 103-176. MR2377247(2009c:55010)

[13] H. Fausk, D.C. Isaksen, t-model structures. Homology, Homotopy Appl. 9 (2007), no. 1, 399-438. MR2299805 (2008d:55013)

[14] P.G. Goerss, Homotopy fixed points for Galois groups. The Čech centennial (Boston, MA, 1993), 187-224, Contemp. Math., 181, Amer. Math. Soc., Providence, RI, 1995. MR1320993 (96a:55008)

[15] P.G. Goerss, H.-W. Henn, M. Mahowald, C. Rezk, A resolution of the $K(2)$-local sphere at the prime 3. Ann. of Math. (2) 162 (2005), no. 2, 777-822. MR2183282 (2006j:55016)

[16] P.G. Goerss, M.J. Hopkins, Moduli spaces of commutative ring spectra. Structured ring spectra, 151-200, London Math. Soc. Lecture Note Ser., 315, Cambridge Univ. Press, Cambridge, 2004. MR2125040 (2006b:55010)

[17] P.S. Hirschhorn, Model Categories and Their Localizations. Mathematical Surveys and Monographs, 99. Amer. Math. Soc., Providence, RI, 2003. MR1944041 (2003j:18018)

[18] M.J. Hopkins, M. Mahowald, H. Sadofsky, Constructions of elements in Picard groups. Topology and representation theory (Evanston, IL, 1992), 89-126, Contemp. Math., 158, Amer. Math. Soc., Providence, RI, 1994. MR1263713 (95a:55020)

[19] M. Hovey, Bousfield localization functors and Hopkins' chromatic splitting conjecture. The Čech centennial (Boston, MA, 1993), 225-250, Contemp. Math., 181, Amer. Math. Soc., Providence, RI, 1995. MR1320994 (96m:55010)

[20] M. Hovey. Spectra and symmetric spectra in general model categories. J. Pure Appl. Algebra 165 (2001), no. 1, 63-127. MR.1860878 (2002j:55006)

[21] M. Hovey, B. Shipley, J. Smith, Symmetric spectra. J. Amer. Math. Soc. 13 (2000), no. 1, 149-208. MR 1695653 (2000h:55016)

[22] M. Hovey, N.P. Strickland, Morava $K$-theories and localisation. Mem. Amer. Math. Soc. 139 (1999), no. 666, viii+100 pp. MR.1601906 (99b:55017)

[23] D.C. Isaksen, Generalized cohomology of pro-spectra. Preprint.

[24] J.F. Jardine, Simplicial presheaves. J. Pure Appl. Algebra 47 (1987), no. 1, 35-87. MR906403 $(88 \mathrm{j}: 18005)$

[25] J.F. Jardine, Stable homotopy theory of simplicial presheaves. Canad. J. Math. 39 (1987), no. 3, 733-747. MR.905753 (88j:18004)

[26] J.F. Jardine, Generalized étale cohomology theories. Progress in Mathematics, 146. Birkhäuser Verlag, Basel, 1997. MR.1437604 (98c:55013) 
[27] J.F. Jardine, Generalised sheaf cohomology theories. Axiomatic, enriched and motivic homotopy theory, 29-68, NATO Sci. Ser. II Math. Phys. Chem., 131, Kluwer Acad. Publ., Dordrecht, 2004. MR2061851 (2005f:55004)

[28] M.A. Mandell, Equivariant symmetric spectra. Homotopy theory: Relations with algebraic geometry, group cohomology, and algebraic $K$-theory, 399-452, Contemp. Math., 346, Amer. Math. Soc., Providence, RI, 2004. MR2066508 (2005d:55019)

[29] M.A. Mandell, J.P. May, S. Schwede, B. Shipley, Model categories of diagram spectra. Proc. London Math. Soc. (3) 82 (2001), no. 2, 441-512. MR1806878 (2001k:55025)

[30] J.P. May, The geometry of iterated loop spaces. Lecture Notes in Mathematics, Vol. 271. Springer-Verlag, Berlin-New York, 1972. MR0420610(54:8623b)

[31] H.R. Miller, On relations between Adams spectral sequences, with an application to the stable homotopy of a Moore space. J. Pure Appl. Algebra 20 (1981), no. 3, 287-312. MR604321 (82f:55029)

[32] S.A. Mitchell, Hypercohomology spectra and Thomason's descent theorem. Algebraic $K$ theory (Toronto, ON, 1996), 221-277, Fields Inst. Commun., 16, Amer. Math. Soc., Providence, RI, 1997. MR1466977 (99f:19002)

[33] C. Rezk, Notes on the Hopkins-Miller theorem. Homotopy theory via algebraic geometry and group representations (Evanston, IL, 1997), 313-366, Contemp. Math., 220, Amer. Math. Soc., Providence, RI, 1998. MR.1642902 (2000i:55023)

[34] J. Rognes, Galois extensions of structured ring spectra. Stably dualizable groups, 1-97, Mem. Amer. Math. Soc. 192 (2008), no. 898. MR2387923 (2009c:55007)

[35] J. Rognes, A Galois extension that is not faithful. Available at the website http://folk.uio. no/rognes/papers/unfaithful.pdf.

[36] S. Schwede, On the homotopy groups of symmetric spectra. Geom. Topol. 12 (2008), no. 3, 1313-1344. MR.2421129 (2009c:55006)

[37] S. Schwede, An untitled book project about symmetric spectra. Preprint.

[38] J.-P. Serre, Cohomologie Galoisienne, 5. éd., rév. et complétée. Lecture Notes in Mathematics, Vol. 5. Springer-Verlag, Berlin-New York, 1994. MR.1324577 (96b:12010)

[39] N.P. Strickland, Gross-Hopkins duality. Topology 39 (2000), no. 5, 1021-1033. MR1763961 (2001d:55006)

[40] P. Symonds, T. Weigel, Cohomology of $p$-adic analytic groups. New Horizons in Pro- $p$ Groups, 349-410, Birkhäuser Boston, Boston, MA, 2000. MR1765127 (2001k:22025)

[41] R.W. Thomason, Algebraic $K$-theory and étale cohomology. Ann. Sci. École Norm. Sup. (4) 18 (1985), no. 3, 437-552. MR826102 (87k:14016)

[42] J.S. Wilson, Profinite groups. The Clarendon Press, Oxford University Press, New York, 1998. MR.1691054 (2000j:20048)

Department of Mathematics, Massachusetts institute of Technology, Cambridge, Massachusetts 02139

Department of Mathematics, University of Louisiana at Lafayette, Lafayette, LOUISIANA 70504 